\title{
Elastic pion-nucleon scattering in chiral perturbation theory: A fresh look
}

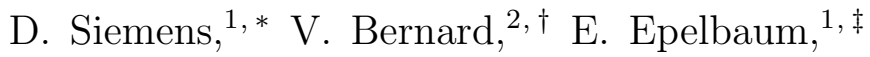 \\ A. Gasparyan, ${ }^{1,3,8}$ H. Krebs,, , and Ulf-G. Meißner ${ }^{4,5,6, * *}$ \\ ${ }^{1}$ Institut für Theoretische Physik II, \\ Ruhr-Universität Bochum, D-44780 Bochum, Germany \\ ${ }^{2}$ Groupe de Physique Théorique, Institut de Physique Nucléaire, \\ UMR 8608, CNRS, Univ. Paris-Sud, \\ Université Paris Saclay, F-91406 Orsay Cedex, France \\ ${ }^{3}$ SSC RF ITEP, Bolshaya Cheremushkinskaya 25, 117218 Moscow, Russia \\ ${ }^{4}$ Institute for Advanced Simulation, \\ Institut für Kernphysik and Jülich Center for Hadron Physics, \\ Forschungszentrum Jülich, D-52425 Jülich, Germany \\ ${ }^{5}$ Helmholtz-Institut für Strahlen- und Kernphysik and Bethe Center for Theoretical Physics, \\ Universität Bonn, D-53115 Bonn, Germany \\ ${ }^{6}$ JARA - High Performance Computing, \\ Forschungszentrum Jülich, D-52425 Jülich, Germany
}

\begin{abstract}
Elastic pion-nucleon scattering is analyzed in the framework of chiral perturbation theory up to fourth order within the heavy-baryon expansion and a covariant approach based on an extended on-mass-shell renormalization scheme. We discuss in detail the renormalization of the various low-energy constants and provide explicit expressions for the relevant $\beta$-functions and the finite subtractions of the power-counting breaking terms within the covariant formulation. To estimate the theoretical uncertainty from the truncation of the chiral expansion, we employ an approach which has been successfully applied in the most recent analysis of the nuclear forces. This allows us to reliably extract the relevant low-energy constants from the available scattering data at low energy. The obtained results provide a clear evidence that the breakdown scale of the chiral expansion for this reaction is related to the $\Delta$-resonance. The explicit inclusion of the leading contributions of the $\Delta$-isobar is demonstrated to substantially increase the range of applicability of the effective field theory. The resulting predictions for the phase shifts are in an excellent agreement with the ones from the recent Roy-Steiner-equation analysis of pion-nucleon scattering.
\end{abstract}

\footnotetext{
*dmitrij.siemens@rub.de †bernard@ipno.in2p3.fr 拉geny.epelbaum@rub.de $\S$ ashot.gasparyan@rub.de hermann.krebs@rub.de *** meissner@hiskp.uni-bonn.de
} 


\section{INTRODUCTION}

Chiral perturbation theory $(\chi \mathrm{PT})$ provides a systematically improvable theoretical framework to analyze low-energy hadronic reactions. It relies on the chiral symmetry of QCD and its breaking patterns, in particular the spontaneous chiral symmetry breaking with the appearance of a triplet of Goldstone bosons, the pions. $\chi \mathrm{PT}$ corresponds to an expansion of the scattering amplitude around the chiral and zero-energy limits. Here, we consider the two-flavor chiral limit with vanishing up and down quark masses and the strange quark mass fixed at its physical value. Characteristic for any effective field theory (EFT), effects of higher energy physics are accounted for via low-energy constants (LECs) accompanying the interaction terms in the effective Lagrangian.

Pioneered in the meson sector [1-3] and extended to the single-baryon [4 7] as well as to few-baryon sectors [8-11], numerous applications and extensions of $\chi \mathrm{PT}$ have been performed over the last decades. Historically, most of the studies in the baryon sector have been carried out utilizing the so-called heavy-baryon (HB) approach [12, 13. In this formulation, the effective chiral Lagrangian is expanded in inverse powers of the nucleon mass treated on the same footing as the breakdown scale of the chiral expansion $\Lambda_{b}$, also referred to as the chiral symmetry breaking scale $\Lambda_{\chi}$. With only negative powers of the nucleon mass appearing in the HB Lagrangian, this formulation offers the simplest way to maintain the power counting for dimensionally regularized loop integrals which enter the scattering amplitude. On the other hand, the strict HB approach does not correctly reproduce certain analytic properties of the scattering amplitude [14-16]. Using manifestly covariant versions of $\chi \mathrm{PT}$ does lead to a correct representation of the analytic properties of the scattering amplitude but requires special care in order to maintain the chiral power counting for loop contributions due to the appearance of positive powers of the nucleon mass $m_{N}$. In the so-called infrared renormalization (IR) scheme proposed by Becher and Leutwyler [16], see also Ref. [17] for a related earlier work, only the infrared-singular (in the limit of vanishing pion masses) pieces of the loop integrals are kept, which are responsible for non-integer powers of the soft scales in the scattering amplitude. On the other hand, the IR scheme of Ref. [16, 18] induces unphysical singularities in the amplitude at high momenta. Alternatively, one may employ the so-called extended on-mass-shell scheme (EOMS) [19, 20] which makes use of the freedom in the choice of renormalization conditions to maintain the chiral power counting. For a detailed discussion and comparison of the various formulations of $\chi \mathrm{PT}$ the reader is referred to Ref. [7].

In this paper we analyze in detail the reaction $\pi N \rightarrow \pi N$ at low energies within the $\mathrm{HB} \chi \mathrm{PT}$ and EOMS formulations at the full one-loop order. Pion-nucleon scattering certainly belongs to the most extensively studied processes in $\chi \mathrm{PT}$, see Refs. [13, 21 30] for the analyses of the elastic channel and Refs. [31 38] for studies of the single-pion production $\pi N \rightarrow \pi \pi N$. It also has attracted renewed interest in recent years in light of its importance for understanding the long-range behavior of the nuclear forces [39 42]. In particular, the state-of-the-art nucleon-nucleon potentials of Ref. [43] include the two-pion exchange contribution derived from the fourth-order approximation of the pion-nucleon scattering amplitude [39]. It was demonstrated in Ref. [43] that nucleon-nucleon scattering data show clear evidence of the resulting two-pion exchange potential, see also Refs. [44, 45] for similar findings at lower chiral orders. Given the ongoing efforts towards pushing the precision frontier in nuclear chiral EFT [46], a reliable determination of pion-nucleon LECs entering the two-pion exchange contributions to the two- and three-nucleon forces with quantified uncertainties 
becomes an important task. This is a non-trivial issue given that most of the $\chi \mathrm{PT}$ studies of pion-nucleon scattering in $\chi \mathrm{PT}$ rely on the Karlsruhe-Helsinki [47] and GWU-SAID [48] partial-wave analyses (PWA) which do not provide information about systematic uncertainties. An important step towards resolving this issue was made recently in Refs. [49, 50], where pion-nucleon scattering was analyzed in the framework of Roy-Steiner equations (RS) and detailed error estimates of all input quantities, the solution procedure and truncations were performed, see Ref. [51] for a review. The resulting phase shifts with quantified uncertainties provide a solid basis for a reliable determination of the LECs. In this paper we, however, follow a different path and analyze directly the available pion-nucleon scattering data at low energies, see also Ref. [40] for a related study. To quantify the theoretical uncertainty from the truncation of the chiral expansion, we employ the approach suggested in Ref. [52] which has also been employed in recent few-nucleon studies [43, 53]. The resulting phase shifts are compared with the ones of Ref. [49] obtained from the Roy-Steiner analysis. We also discuss the role of the $\Delta(1232)$ resonance in this reaction.

Our paper is organized as follows. In section II] the necessary definitions for a study of $\pi N \rightarrow \pi N$ in the HB and covariant approach are given. The renormalization procedures in both chiral approaches are discussed in section III, whereas the details of the fitting procedure can be found in section IV. Our predictions for observables not used in the fitting procedure are collected in section $\overline{\mathrm{V}}$ which also provides a discussion of the obtained results. Next, the explicit inclusion of the lowest-order $\Delta(1232)$ contributions is presented in section VI. Finally, the main results of our study are summarized in section VII. The appendix contains explicit expressions for the renormalized LECs.

\section{BASIC DEFINITIONS}

In this section, we provide some basic definitions which are necessary for the description of the reaction $\pi N \rightarrow \pi N$. The reader familiar with this is invited to skip this section. Throughout this work, the kinematical variables are defined as follows:

$$
\pi^{a}(q) N\left(p=m_{N} v+k\right) \rightarrow \pi^{b}\left(q^{\prime}\right) N^{\prime}\left(p^{\prime}=m_{N} v+k^{\prime}\right)
$$

where $N$ denotes a nucleon and $\pi^{a}$ a pion with the isospin quantum number $a$. Note that the decomposition of the nucleon four-momenta in terms of the four-velocity $v_{\mu}$ and the residual small momentum $k_{\mu}$ is only relevant for the heavy baryon approach. To relate the T-matrix to phase shifts, we follow the procedure of Ref. [54] (Ref. [22]) for the covariant (HB) approach as described below.

\section{A. Covariant chiral perturbation theory}

In the covariant approach, the $T$-matrix can be decomposed in the following way

$$
T^{b a}=\chi_{N^{\prime}}^{\dagger}\left(\delta^{a b} T^{+}+\mathrm{i} \epsilon^{b a c} \tau_{c} T^{-}\right) \chi_{N},
$$

where

$$
T^{ \pm}=\bar{u}^{\left(s^{\prime}\right)}\left(A^{ \pm}+\not B^{ \pm}\right) u^{(s)}
$$

and the amplitudes $A^{ \pm}$and $B^{ \pm}$depend on the Mandelstam variables

$$
s=(p+q)^{2}, \quad t=\left(q-q^{\prime}\right)^{2}, \quad u=\left(p^{\prime}-q\right)^{2}, \quad s+t+u=2 m_{N}^{2}+2 M_{\pi}^{2} .
$$


The partial wave amplitudes can be expressed in terms of $A^{ \pm}$and $B^{ \pm}$as follows:

$$
\begin{aligned}
f_{l \pm}^{I}(s)= & \frac{1}{16 \pi \sqrt{s}}\left(\left(E+m_{N}\right)\left(A_{l}^{I}(s)+\left(\sqrt{s}-m_{N}\right) B_{l}^{I}(s)\right)\right. \\
& \left.+\left(E-m_{N}\right)\left(-A_{l \pm}^{I}(s)+\left(\sqrt{s}+m_{N}\right) B_{l \pm}^{I}(s)\right)\right)
\end{aligned}
$$

where for $X \in\{A, B\}$

$$
X_{l}^{I}(s)=\int_{-1}^{+1} \mathrm{~d} z X^{I}(s, t) P_{l}(z)
$$

with $t=-2 \boldsymbol{q}^{2}(1-z), E=\sqrt{m_{N}^{2}+\boldsymbol{q}^{2}}$ and the relations to the isospin basis read

$$
X^{I=1 / 2}=X^{+}+2 X^{-}, \quad X^{I=3 / 2}=X^{+}-X^{-} .
$$

The phase shifts are obtained by using the unitarization prescription

$$
\delta_{l \pm}^{I}(s)=\arctan \left(|\boldsymbol{q}| \Re f_{l \pm}^{I}(s)\right) .
$$

\section{B. Heavy-baryon chiral perturbation theory}

In the HB approach, the decomposition reads

$$
T^{b a}=\chi_{N^{\prime}}^{\dagger}\left(\delta^{a b} T^{+}+\mathrm{i} \epsilon^{b a c} \tau_{c} T^{-}\right) \chi_{N}
$$

where

$$
T^{ \pm}=\bar{u}_{v}^{\left(s^{\prime}\right)}\left(g^{ \pm}+2 \mathrm{i} \boldsymbol{S} \cdot \boldsymbol{q} \times \boldsymbol{q}^{\prime} h^{ \pm}\right) u_{v}^{(s)} .
$$

The amplitudes $g^{ \pm}$and $h^{ \pm}$depend on the four momenta $k, k^{\prime}, q, q^{\prime}$ and are related to the partial wave amplitudes via

$$
f_{l \pm}^{I}(s)=\frac{E+m_{N}}{16 \pi \sqrt{s}} \int_{-1}^{+1} \mathrm{~d} z\left(g^{I} P_{l}(z)+\boldsymbol{q}^{2} h^{I}\left(P_{l \pm}(z)-z P_{l}(z)\right)\right) .
$$

The relation to the isospin basis is the same as in Eq. (7) with $X \in\{g, h\}$.

\section{Observables}

The observables of interest are differential cross sections $\mathrm{d} \sigma / \mathrm{d} \Omega$ and polarizations $P$ for the three channels $\pi^{+} p \rightarrow \pi^{+} p, \pi^{-} p \rightarrow \pi^{-} p$ and $\pi^{-} p \rightarrow \pi^{0} n$. At low energy and/or forward angles, these observables are strongly affected by electromagnetic interactions which are taken into account following the procedure described in Ref. [55]. This paper also provides all the necessary formula to relate the strong phase shifts in Eq. (8) to the observables we are interested in. Still, it should be understood that the treatment of the electromagnetic effects in that paper is approximative. 


\section{POWER COUNTING AND RENORMALIZATION}

In $\chi \mathrm{PT}$, the invariant amplitudes are calculated in the chiral expansion with the expansion parameter

$$
Q=\left\{\frac{q}{\Lambda_{b}}, \frac{M_{\pi}}{\Lambda_{b}}\right\}
$$

where $M_{\pi}$ is the pion mass, $q$ denotes generic three- (four-) momenta of external nucleons (pions) and $\Lambda_{b}$ is the breakdown scale of the chiral expansion whose value will be specified below. Since the nucleon mass $m_{N}$ does not vanish in the chiral limit, the power counting employed in the Goldstone boson sector breaks down for dimensionally regularized loop integrals in the presence of baryons. The traditional way of curing this problem is the HB approach [12, 13], where the nucleon mass is treated as an additional large scale, $m_{N} \sim \Lambda_{b}$, and a $1 / m_{N}$ expansion is performed at the level of the effective Lagrangian. For certain observables such as some of the nucleon form factors, the HB expansion exhibits a very limited rage of convergence [15, 16, ${ }^{1}$. It is, therefore, advantageous to employ the Lorentz covariant formulations of baryon $\chi \mathrm{PT}$ using either the IR [16] or the EOMS scheme [19, 20] in order to maintain the power counting. In this work, we will employ the HB and covariant EOMS approaches. In both schemes, the effective Lagrangian needed to describe pionnucleon dynamics at one-loop level consists of the following pieces (see Ref. [24] for a full list of terms):

$$
\mathcal{L}_{\text {eff }}=\mathcal{L}_{\pi \pi}^{(2)}+\mathcal{L}_{\pi \pi}^{(4)}+\mathcal{L}_{\pi N}^{(1)}+\mathcal{L}_{\pi N}^{(2)}+\mathcal{L}_{\pi N}^{(3)}+\mathcal{L}_{\pi N}^{(4)}
$$

where the superscripts refer to the chiral dimension. Further, for the HB approach, we will also show results corresponding to the power counting assignment $m_{N} \sim \Lambda_{b}^{2} / M_{\pi}$, which is commonly used in the studies of the nuclear forces [10] and will be referred to as HB-NN. The above assignment results in the relativistic corrections being pushed to higher orders in the EFT expansion as compared to the standard HB approach used in the single-baryon sector, which will be referred to as $\mathrm{HB}-\pi \mathrm{N}$.

Before discussing the renormalization of the $\pi N \rightarrow \pi N$ amplitudes, we need to express the bare quantities in the leading-order Lagrangian in terms of physical ones. The expressions for $m_{N}$ and the nucleon axial vector coupling $g_{A}$ for both chiral approaches are given in Appendix A. Throughout this work, we express all results in terms of the effective axial vector coupling constant $g_{A}$ which takes into account the Goldberger-Treiman discrepancy and is related to the physical axial vector coupling $g_{A, p h}$ via

$$
g_{A}=g_{A, p h}-2 M_{\pi}^{2} d_{18}+\mathcal{O}\left(Q^{5}\right) .
$$

The value of $g_{A}$ is fixed by the Goldberger-Treiman relation

$$
g_{A}=\frac{g_{\pi N N} F_{\pi}}{m_{N}} .
$$

For the pion-nucleon coupling constant $g_{\pi N N}$, we adopt the value from Ref. [56], $g_{\pi N N}^{2} / 4 \pi=$ 13.7(2) leading to $g_{A}=1.289(1)$. Note that we do not study the effects of the uncertainty of

\footnotetext{
${ }^{1}$ It should, however, be noted that these deformations of the analytic structure of the underlying amplitudes can be overcome easily by including the first $1 / m_{N}$ correction into the heavy fermion propagator, $i /(v \cdot k) \rightarrow i /\left(v \cdot k+k^{2} /\left(2 m_{N}\right)\right)$.
} 
$g_{A}$ in this work and only employ the mean value. In addition to removing the redundant (for the considered reaction) LEC $d_{18}$, using $g_{A}$ ensures a correct reproduction of the analytic structure of the $\pi N \rightarrow \pi N$ scattering amplitude.

The relevant tree-level diagrams for $\pi N \rightarrow \pi N$ to order $Q^{4}$ are visualized in Fig. 1 while the leading-order loop diagrams are shown in Fig. 2. The next-to-leading order loop diagrams are not shown explicitly but can be easily generated by replacing one of the lowest-order $\pi N$-vertices with an even number of pions in the shown loop diagrams by a subleading one from $\mathcal{L}_{\pi N}^{(2)}$ as visualized in Fig. 3. Notice that there are no $\pi N$-vertices with an odd number of pions in $\mathcal{L}_{\pi N}^{(2)}$.

The leading-order tree-level diagrams are constructed solely from the lowest-order vertices and thus depend only on the well-known LECs $F_{\pi}$ and $g_{A}$. The higher-order tree-level graphs involve insertions of vertices with the LECs $c_{i}$ from $\mathcal{L}_{\pi N}^{(2)}, d_{i}$ from $\mathcal{L}_{\pi N}^{(3)}, e_{i}$ from $\mathcal{L}_{\pi N}^{(4)}$ and the purely mesonic LECs $l_{i}$ from $\mathcal{L}_{\pi \pi}^{(4)}$. Some of the LECs $e_{i}$ enter the $\pi N$ scattering amplitude only within linear combinations with the LECs $c_{i}$. In order to get rid of the redundant LECs, we make the following redefinitions on the level of the renormalized LECs discussed below [25]

$$
\begin{aligned}
& \bar{c}_{1} \rightarrow \bar{c}_{1}+2 M_{\pi}^{2}\left(\bar{e}_{22}-4 \bar{e}_{38}+\bar{c}_{1} \beta_{l_{3}} \bar{l}_{3} /\left(32 \pi^{2} F_{\pi}^{2}\right)\right), \\
& \bar{c}_{2} \rightarrow \bar{c}_{2}-8 M_{\pi}^{2}\left(\bar{e}_{20}+\bar{e}_{35}\right), \\
& \bar{c}_{3} \rightarrow \bar{c}_{3}-4 M_{\pi}^{2}\left(2 \bar{e}_{19}-\bar{e}_{22}-\bar{e}_{36}\right), \\
& \bar{c}_{4} \rightarrow \bar{c}_{4}-4 M_{\pi}^{2}\left(2 \bar{e}_{21}-\bar{e}_{37}\right) .
\end{aligned}
$$

This is a general phenomenon in $\chi \mathrm{PT}$, namely that working an sufficiently high orders, one encounters quark mass renormalizations of certain lower order LECs that can not be resolved for the physical values of the quark masses. Finally, the $\pi N$-scattering amplitudes depend on the LECs $c_{1,2,3,4}, d_{1+2,3,5,14-15}$ and $e_{14,15,16,17,18}$. This number is consistent with the most general polynomial representation of the $\pi N$ scattering amplitude to fourth order, see e.g. Ref. [57].

The renormalization of the LECs in the HB formalism can be performed order-by-order in a complete analogy with the mesonic sector, where one has (using dimensional regularization)

$$
l_{i}=\frac{\beta_{l_{i}}}{32 \pi^{2}} \bar{l}_{i}+\beta_{l_{i}}\left(\bar{\lambda}+\frac{1}{32 \pi^{2}} \log \left(\frac{M_{\pi}^{2}}{\mu^{2}}\right)\right)
$$

with

$$
\bar{\lambda}=\frac{\mu^{d-4}}{16 \pi^{2}}\left(\frac{1}{d-4}+\frac{1}{2}\left(\gamma_{E}-1-\ln 4 \pi\right)\right) .
$$

The ultraviolet (UV) divergent pieces in the HB scattering amplitude up to order $Q^{4}$ are canceled by the counter terms upon expressing the bare LECs $d_{i}$ and $e_{i}$ in terms of the renormalized ones $\bar{d}_{i}$ and $\bar{e}_{i}$ via

$$
\begin{aligned}
& d_{i}=\bar{d}_{i}+\frac{\beta_{d_{i}}}{F_{\pi}^{2}}\left(\bar{\lambda}+\frac{1}{32 \pi^{2}} \log \left(\frac{M_{\pi}^{2}}{\mu^{2}}\right)\right) \\
& e_{i}=\bar{e}_{i}+\frac{\beta_{e_{i}}}{F_{\pi}^{2}}\left(\bar{\lambda}+\frac{1}{32 \pi^{2}} \log \left(\frac{M_{\pi}^{2}}{\mu^{2}}\right)\right)
\end{aligned}
$$

where the relevant $\beta$-functions are listed in Appendix B. For the LECs $d_{i}$, the $\beta$-functions are identical to those of Refs. [58, see also [22]. For the LECs $e_{i}$, we have verified that the 
obtained $\beta$-functions are identical to the ones listed in Ref. [16] after changing their operator basis to ours. Note that $c_{i}=\bar{c}_{i}$ in the HB framework.

In the covariant approach, the renormalization of the LECs is more complicated. After performing dimensional regularization with the $\overline{\mathrm{MS}}$ scheme, loop diagrams still contribute at every chiral order which violates the power counting. The main idea to resolve this issue is based on the observation that a loop function can be split into an IR regular and IR singular parts. All power counting breaking terms (PCBTs) stemming from loop graphs are included in the IR regular part, which is analytic in the quark mass and momenta in $d$ dimension and thus can be absorbed into LECs of the most general Lagrangian [19, 20. For our purpose we need to consider the IR regular parts from the loop graphs of order $Q^{3}$ and $Q^{4}$ in the naive counting which, after renormalization of the leading-order couplings $m_{N}$ and $g_{A}$, start to appear at order $Q^{2}$. Therefore, we perform an additional finite renormalization of the LECs as follows

$$
\begin{aligned}
c_{i} & =\bar{c}_{i}+\delta c_{i}^{(3)}+\delta c_{i}^{(4)} \\
d_{i} & =\bar{d}_{i}+\delta d_{i}^{(3)}+\delta d_{i}^{(4)} \\
e_{i} & =\bar{e}_{i}+\delta e_{i}^{(4)}
\end{aligned}
$$

where for $x \in\{c, d, e\}$

$$
\begin{aligned}
\delta x_{i}^{(n)} & =\frac{\delta \bar{x}_{i, f}^{(n)}}{F_{\pi}^{2}}+\frac{\beta_{x_{i}, B}^{(n)}}{F_{\pi}^{2}}\left(\bar{\lambda}+\frac{1}{32 \pi^{2}} \log \left(\frac{m_{N}^{2}}{\mu^{2}}\right)\right)+\frac{\beta_{x_{i}, M}^{(n)}}{F_{\pi}^{2}}\left(\bar{\lambda}+\frac{1}{32 \pi^{2}} \log \left(\frac{M_{\pi}^{2}}{\mu^{2}}\right)\right) \\
& =\frac{\delta \bar{x}_{i, f}^{(n)}}{F_{\pi}^{2}}+\frac{\beta_{x_{i}}^{(n)}}{F_{\pi}^{2}}\left(\bar{\lambda}+\frac{1}{32 \pi^{2}} \log \left(\frac{m_{N}^{2}}{\mu^{2}}\right)\right)+\frac{\beta_{x_{i}}}{32 F_{\pi}^{2} \pi^{2}} \log \left(\frac{M_{\pi}^{2}}{m_{N}^{2}}\right) .
\end{aligned}
$$

Here, $\delta \bar{x}_{i, f}^{(n)}$ denotes the negative of the finite IR regular parts from loops of naive order $n$, while $\beta_{x_{i}, B}^{(n)}$ and $\beta_{x_{i}, M}^{(n)}$ are the $\beta$-functions which are needed to cancel the baryonic and mesonic tadpoles, respectively. In order to make the notation more compact, we made the replacements $\beta_{x_{i}}^{(n)}=\beta_{x_{i}, B}^{(n)}+\beta_{x_{i}, M}^{(n)}$ and $\beta_{x_{i}}=\beta_{x_{i}, M}^{(n)}$, with $\beta_{x_{i}}$ from Eq. (19) in the last line of the above equation. Note that in Eq. (20), we absorb all IR regular pieces up to the order we are working at. This procedure does, strictly speaking, differ from the EOMS approach where only PCBTs are absorbed into the LECs. In EOMS at order $Q^{3}\left(Q^{4}\right)$, one would only absorb the IR regular pieces up to the order $Q^{2}\left(Q^{3}\right)$, instead we absorb them up to the order $Q^{3}\left(Q^{4}\right)$. In addition, we also perform shifts of the LECs proportional to $\log \left(M_{\pi}^{2} / m_{N}^{2}\right)$, which is not done in EOMS. This modified version of the EOMS is employed in this work to guarantee the equivalence between the results in the HB and covariant approaches up to the order we are working with the difference being of higher orders only. Thus, an expansion of our renormalized covariant amplitudes at orders $Q^{3}$ and $Q^{4}$ in inverse powers of the nucleon mass $m_{N}$ would give our renormalized HB amplitudes up to order $Q^{3}$ and $Q^{4}$, respectively. Note that our renormalized amplitudes are equivalent to $\pi N \rightarrow \pi N$ amplitudes renormalized in EOMS. There is no loss of information, just a reshifting of terms from the amplitude to the LECs.

We have determined the finite and UV divergent pieces in the following way. First, we have changed the basis for the scattering amplitude such that every spin structure fullfills the power counting by itself leading to [26]

$$
\left.T^{ \pm}=\bar{u}^{\left(s^{\prime}\right)}\left(D^{ \pm}-\frac{1}{4 m_{N}}\left[\phi^{\prime}, \not\right]\right] B^{ \pm}\right) u^{(s)} .
$$


where $D=A+\nu B$ with $\nu=(s-u) /\left(4 m_{N}\right)$. Next, $D$ and $B$ are expanded in small parameters

$$
M_{\pi} \sim \mathcal{O}\left(Q^{1}\right), \quad s-m_{N}^{2} \sim \mathcal{O}\left(Q^{1}\right), \quad u-m_{N}^{2} \sim \mathcal{O}\left(Q^{1}\right), \quad t \sim \mathcal{O}\left(Q^{2}\right)
$$

Note that while the linear combination $s+u-2 m_{N}^{2}$ counts according to the above estimations as order- $Q^{1}$, it actually starts contributing only at order $Q^{2}$ due to the cancellation of the order- $Q^{1}$ terms, see e.g. Eq. (4). Therefore, for practical reasons, it is advantageous to express $D$ and $B$ either in $(s, t)$ or $(u, t)$. Also note that $m_{N}$ in Eq. (23) denotes the physical nucleon mass, whereas the expansion in the EOMS scheme is, strictly speaking, around the nucleon mass in the chiral limit $\stackrel{\circ}{n}_{N}$. The difference is of the order of $m_{N}-\grave{m}_{N} \sim \mathcal{O}\left(Q^{2}\right)$ and is thus affecting the shifts at chiral order $Q^{4}$. However, due to our choice to work with the shifted LECs $c_{i}$, see Eq. (16), this amounts merely to a reshuffling of the terms between the $c_{i}$ and $e_{i}$ and does not affect the final results.

The pertinent $\beta$-functions can be calculated by substituting every loop function by its UV divergent part and expanding the result in small parameters. The determination of the finite IR regular pieces is more demanding. It requires the substitution of the loop functions by their IR regular parts. This has been achieved by interchanging the loop integration with a Taylor series in powers of the small parameters.

Several checks on the $\pi N \rightarrow \pi N$ amplitudes have been performed. The renormalization of $m_{N}, Z_{N}$ and $g_{A}$ was checked by setting the internal nucleon line in the covariant (heavy baryon) $\pi N$ amplitudes on-shell. An expansion around $s=m_{N}^{2}$ or $u=m_{N}^{2}$ corresponding to vanishing pion energy in the center-of-mass system (CMS), $\omega=0$, showed that only the leading order diagrams exhibit poles and thus giving the right analytic structure of the amplitudes. Using the redefined LECs from Appendix B, the $\pi N \rightarrow \pi N$ amplitudes fullfil power-counting and are UV-finite up to order $Q^{3}$ and $Q^{4}$, respectively. Another consistency check was done by using the same renormalization shifts in the amplitudes of the reaction $\pi N \rightarrow \pi \pi N$, whose analysis will be published elsewhere, and verifying the power counting and UV-finiteness by redefining only the new LECs appearing in $\pi N \rightarrow \pi \pi N$. In Appendix $\mathrm{B}$ we list all LECs appearing in both reactions. The pion field was defined in the most general form given by unitarity

$$
U=1+\mathrm{i} \frac{\boldsymbol{\tau} \cdot \boldsymbol{\pi}}{F_{\pi}}-\frac{\boldsymbol{\pi}^{2}}{2 F_{\pi}^{2}}-\mathrm{i} \alpha \frac{\boldsymbol{\pi}^{2} \boldsymbol{\tau} \cdot \boldsymbol{\pi}}{F_{\pi}^{3}}+\frac{(8 \alpha-1)}{8 F_{\pi}^{4}} \boldsymbol{\pi}^{4}+\ldots
$$

and it was checked that the final renormalized amplitudes are independent of the parameter $\alpha$. We checked our amplitudes by comparing them with the results of Ref. [30]. Notice that the expressions published in that reference contain some typos. We, however, were able to reproduce their results by comparing the explicit expressions in a Mathematica notebook with the ones provided by one of the authors of Ref. [30]. To avoid the same problems with typing rather lengthy expressions, we prefer to provide the amplitudes in a Mathematica notebook upon request.

Finally, we emphasize that we take the isospin limit in all our amplitudes, i.e. we take $m_{p}=m_{n}=m_{N}$ and $M_{\pi^{ \pm}}=M_{\pi^{0}}=M_{\pi}$. The electromagnetic corrections of Ref. [55] employed in our analysis do, of course, take into account some of the isospin-breaking effects. However, it is also clear that this procedure does not include all possible isospin violating effects. For a fully consistent calculation including all such effects for the $\pi N$ scattering lengths, see e.g. Ref. [59]. 


\section{FITTING PROCEDURE}

The amplitudes for the reaction $\pi N \rightarrow \pi N$ depend on several LECs as explained in section III. Throughout this work, we use the following values for the various LECs and masses entering the leading order effective Lagrangian: $M_{\pi}=139.57 \mathrm{MeV}, F_{\pi}=92.2 \mathrm{MeV}$, $m_{N}=938.27 \mathrm{MeV}$ [60]. All LECs should be understood as renormalized quantities as discussed in the previous section. For convenience, we will suppress in the following the bars on the renormalized LECs $\bar{c}_{i}, \bar{d}_{i}$ and $\bar{e}_{i}$, which values are always given in units of $\mathrm{GeV}^{-1}$, $\mathrm{GeV}^{-2}$ and $\mathrm{GeV}^{-3}$, respectively.

All fits described below are performed to $\pi N \rightarrow \pi N$ scattering data $\mathrm{d} \sigma / \mathrm{d} \Omega, P$ in all three channels simultaneously. In this least squares fit we minimize the quantity

$$
\chi^{2}=\sum_{i}\left(\frac{\mathcal{O}_{i}^{\exp }-N_{i} \mathcal{O}_{i}^{(n)}}{\delta \mathcal{O}_{i}}\right)^{2} \quad \text { with } \quad \delta \mathcal{O}_{i}=\sqrt{\left(\delta \mathcal{O}_{i}^{\exp }\right)^{2}+\left(\delta \mathcal{O}_{i}^{(n)}\right)^{2}}
$$

where $\mathcal{O}_{i}^{e x p}, \delta \mathcal{O}_{i}^{e x p}$ and $N_{i}$ are taken from the GWU-SAID data base 61] and $\mathcal{O}_{i}^{(n)}$ denotes the observable calculated in $\chi \mathrm{PT}$ up to order $n$. The theoretical error takes into account the uncertainty from the truncation of the chiral expansion at a given order and is estimated in the way proposed in Ref. [52], namely

$$
\delta \mathcal{O}_{i}^{(n)}=\max \left(\left|\mathcal{O}_{i}^{(\mathrm{LO})}\right| Q^{n-\mathrm{LO}+1},\left\{\left|\mathcal{O}_{i}^{(k)}-\mathcal{O}_{i}^{(j)}\right| Q^{n-j}\right\}\right) \quad \text { with } \quad j<k \leq n
$$

and $Q=\omega_{C M S} / \Lambda_{b}$, where $\omega_{C M S}$ denotes the energy of the incoming pion in the CMS frame. Further, LO refers to the chiral order, at which the observable $\mathcal{O}_{i}$ appears receives its first nonvanishing contribution. In the Goldstone boson and single-baryon sectors, the breakdown scale of the chiral expansion is often assummed to be of the order of $\Lambda_{b} \sim \Lambda_{\chi} \sim$ $M_{\rho} \sim 4 \pi F_{\pi} \sim 1 \mathrm{GeV}$. On the other hand, a somewhat more conservative estimation of $\Lambda_{b} \sim 600 \mathrm{MeV}$ was obtained and employed in a recent study of nucleon-nucleon scattering in Ref. [52]. It was also verified in an analysis of Ref. [62] utilizing the Bayesian approach. Here and in what follows, we adopt the more conservative estimate of $\Lambda_{b} \sim 600 \mathrm{MeV}$ which seems to be justified given the implicit inclusion of the Roper resonance in our calculations. In addition to Eq. (26), the theoretical errors is required to be at least of the size of actual higher-order contribution

$$
\delta \mathcal{O}_{i}^{(n)} \geq \max \left(\left\{\left|\mathcal{O}_{i}^{(k)}-\mathcal{O}_{i}^{(j)}\right|\right\}\right) \quad \text { with } \quad n \leq j<k .
$$

Both Eqs. (26) and (27) are implemented in the fits using an iterative procedure. ${ }^{2}$

To give a meaningful uncertainty quantification for other observables we define the correlation and covariance matrices as follows

$$
\begin{aligned}
\operatorname{Cov}\left(c_{i} c_{j}\right) & =\mathbf{H}_{i j}^{-1} \quad \text { with } \quad \mathbf{H}_{i j}=\left.\frac{1}{2} \frac{\partial^{2} \chi^{2}}{\partial c_{i} \partial c_{j}}\right|_{\boldsymbol{c}=\boldsymbol{c}_{*}}, \\
\operatorname{Corr}\left(c_{i} c_{j}\right) & =\operatorname{Cov}\left(c_{i} c_{j}\right) / \sqrt{\operatorname{Cov}\left(c_{i} c_{i}\right) \operatorname{Cov}\left(c_{j} c_{j}\right)},
\end{aligned}
$$

where $\boldsymbol{c}$ is a set of LECs and $\boldsymbol{c}_{*}$ is the set which minimizes $\chi^{2}$. The correlation and covariance matrices for the fits discussed above are given in Tables II and III. Note the correlations at order $Q^{4}$ between $c_{1}$ and $c_{2}$ and the additional correlations in the $\mathrm{HB}$ countings between $c_{2}$ and $e_{16}$ and between $c_{4}$ and $d_{1+2}$.

\footnotetext{
${ }^{2}$ As a starting point in this iterative procedure, we performed fits without theoretical errors.
} 


\section{FIT RESULTS, PREDICTIONS AND DISCUSSION}

We performed fits to all available data for all scattering angles and an incoming pion kinetic energy $T_{\pi}<\{50,75,100,125,150\} \mathrm{MeV}$, which corresponds to $\{1035,1368,1704,1854,2176\}$ data points, respectively. In the upper panel of Fig. 5, we show a representative fit to $\mathrm{d} \sigma / \mathrm{d} \Omega$ for the channel $\pi^{+} p \rightarrow \pi^{+} p$ at $T_{\pi}=43.3 \mathrm{MeV}$. A precise definition of the uncertainty bands will be given below. The fitted LECs as a function of the maximal fitting energy $T_{\pi}$ are shown in Figs. 7 and 8 while the reduced $\chi^{2}\left(\bar{\chi}^{2}\right)$ with (without) theoretical errors as a function of $T_{\pi}$ is plotted in Fig. 6. As can be seen in the figures, most of the fitted LECs exhibit a plateau-like behavior for the maximal fitting energy in the range between $75 \mathrm{MeV}$ and $125 \mathrm{MeV}$ yielding, at the same time, a reasonable reduced $\chi^{2}$ close to 1 . On the other hand, the $\chi^{2} /$ dof starts increasing when experimental data at higher energies are included in contradiction with an expected flat behavior. This feature is also reflected in the deviation of the LECs viewed as functions of $T_{\pi}$ from a plateau-like behavior when higher-energy data are included in the fit as visualized in Figs. 7 and 8 . The observed instability of the fits at higher energies provide a clear indication that the actual theoretical uncertainty is larger than the one estimated as described in the previous section. As will be shown below, the slow convergence pattern of the chiral expansion is caused by the $\Delta(1232)$ resonance which is not explicitly included in the considered formulations of $\chi \mathrm{PT}$.

The extracted values of the LECs at orders $Q^{2}, Q^{3}, Q^{4}$ are listed in Table I for all considered approaches along with the corresponding values of the reduced $\chi^{2}$ and $\bar{\chi}^{2}$. For the sake of compactness, we restrict ourselves here and in what follows to the fits with $T_{\pi}<100 \mathrm{MeV}$ which can be regarded as representative examples. As expected, the value of $\bar{\chi}^{2} /$ dof decreases with an increasing chiral order showing the improved description of the data. Notice further that all considered approaches lead to a similar quality of the fits. The extracted values of the LECs do not show a strong dependence on the counting scheme except for some of the $e_{i}$ 's at order $Q^{4}$ and are generally in a reasonably good agreement with the values reported in the literature. Specifically, except for the value of $c_{2}$, the LECs $c_{i}$ and $d_{i}$ extracted at order $Q^{4}$ in the HB-NN approach are compatible with the ones determined in Ref. [39] from the KH and GW-SAID PWA if the spread between the results based on the two different PWA is interpreted as the uncertainty. The large differences in the values of the LECs $c_{2}$ and $e_{16}$ are naturally explained by the very strong correlation between these LECs, see Table III. On the other hand, it is comforting to see that the LECs $e_{14}$ and $e_{17}$ which enter the order- $Q^{5}$ contribution to the three-nucleon force [39] are rather stable. Similar conclusions apply to a comparison with the recent determination of the LECs from the subthreshold coefficients obtained in the RS analysis [50], although the differences between the LECs generally appear to be somewhat larger. In any case, the sizable (large) shifts in the LECs $c_{i}\left(d_{i}\right)$ extracted at different orders in the chiral expansion indicate that the uncertainties in their values are presently dominated by the truncation of the chiral expansion.

We are now in the position to discuss predictions of other observables not used in the fits. Here and in what follows, we will use the values of the LECs collected in Table I. All predictions are supplemented with an estimated uncertainty which includes both the statistical and theoretical errors. Here and in what follows, the error associated with the uncertainty in the values of the LECs determined by the fitting procedure specified in the 
previous section will be referred to as statistical. It is calculated via

$$
\left(\delta \mathcal{O}_{i}^{\text {stat }}\right)^{2}=\boldsymbol{J}^{T} \mathbf{H}^{-1} \boldsymbol{J} \quad \text { with } \quad J_{j}=\left.\frac{\partial \mathcal{O}_{i}}{\partial c_{j}}\right|_{c=c_{*}}
$$

whereas the theoretical uncertainty from the truncation of the chiral expansion is estimated using Eqs. (26) and (27) using the central values of the LECs determined in a corresponding fit.

The predicted phase shifts in the $S, P, D$ and $F$ partial waves with pion energies up to $100 \mathrm{MeV}$ at orders $Q^{2}, Q^{3}$ and $Q^{4}$ are shown in Figs. 9 - 14 for all three considered formulations of $\chi \mathrm{PT}$ in comparison with the phase shifts from the RS results of Ref. [49] for $S$ - and $P$-waves and with the GWU-SAID solution [48, 64] for $D$ - and $F$-waves. Given that for predictions we use the same definition of the theoretical error as employed in the fits, the statistical and theoretical uncertainties for a predicted quantity are not really independent from each other and it is not clear to us how to combine them in a meaningful way. For this reason, we will show in the following both kinds of uncertainties separately. The extracted phase shifts in the $S$ and $P$ waves shown in Figs. 9 and 10 agree with the RS results for energies up to $T_{\pi}<70 \mathrm{MeV}$. For energies above $70 \mathrm{MeV}$, the difference between the $Q^{3}$ and $Q^{4}$ predictions increases which results in rather large theoretical uncertainties. This applies especially to the $P_{11}$ partial wave which is not surprising given the smallness of the corresponding phase shift. On the other hand, the statistical uncertainties appear to be negligibly small for the $S$ and $P$ waves. One also observes that all considered formulations lead to nearly identical results for these phase shifts which is consistent with the similar values of $\bar{\chi}_{\pi N}^{2} /$ dof, see Table I.

The situation is rather different for the $D$ waves which are shown in Figs. 11 and 12 in comparison with the results of the GWU-SAID partial wave analysis. Note that the GWUSAID PWA does not provide an uncertainty for their phase shifts so that a comparison with our predictions should be taken with care. Similarly to the $S$ - and $P$-waves, one observes large shifts between the order- $Q^{3}$ and $Q^{4}$ predictions which result in a very large theoretical uncertainty at order $Q^{3}$. Statistical errors appear to be completely negligible at this order. At order $Q^{3}$, our predictions are consistent with the GWU-SAID PWA (within the very large theoretical uncertainties). However, at the highest considered order $Q^{4}$, our results do show significant disagreements with the GWU-SAID PWA especially in the $D_{35}$ partial wave for the HB-NN counting and $D_{33}$ and $D_{15}$ partial waves for the covariant approach, see Fig. 12. We, however, emphasize that the statistical uncertainty is not negligible anymore at this order. It stems mainly from correlations, see Table III, as well as from the relatively large uncertainty in the determined values of the LECs $e_{i}$. Our predictions for $F$-waves are visualized in Figs. 13 and 14 and show a better agreement with the GWU-SAID PWA except for the HB-NN scheme.

We also show in Table VII the predictions for the threshold and subtreshold parameters in comparison with the values from the RS-analysis [50], see also Refs. [56, 65]. For the subthreshold and threshold region we used $Q=M_{\pi} / \Lambda_{b}$ as expansion parameter in the theoretical error in Eq. (26). We calculated the subthreshold parameters and scattering lengths in all three counting schemes. We reproduced the analytic expressions in Ref. [26] for the HB formulation. The covariant expressions are lengthy and can be provided upon request. While the predictions at $Q^{2}, Q^{3}$ are mostly in agreement with the empirical values within uncertainties, the results at order $Q^{4}$ do exhibit significant discrepancies in many cases. Furthermore, the $Q^{4}$ results show often no improvement compared with the $Q^{3}$ ones. 
The above findings within the HB-NN, HB- $\pi \mathrm{N}$ and the covariant approaches appear to be not quite satisfactory in the following respects:

- The resulting $\chi^{2} /$ dof is found to increase if scattering data at energies above $T_{\pi} \sim$ $100 \mathrm{MeV}$ are included in the fits in contradiction with the expected nearly constant behavior.

- There are deviations from a plateau-behavior for the extracted LECs as a function of the maximal fitting energy which indicates that the fits become unstable if the energy is increased.

- One observes large disagreements between the predicted $D$-wave phase shifts and the results of the GWU-SAID PWA at order $Q^{4}$.

- Large deviations are observed for some of the predicted subthreshold coefficients at order $Q^{4}$.

These inconsistencies indicate that the actual breakdown scale of the chiral expansion in our calculations is smaller than the assumed $\Lambda_{\chi} \simeq 600 \mathrm{MeV}$ and, as a result, that the theoretical uncertainty has been underestimated. Given that the results are similar for all considered approaches, there is no indication that the slow convergence of the chiral expansion is to be attributed to the treatment of relativistic corrections. Clearly, the most natural explanation of the observed pattern is provided by the $\Delta(1232)$ resonance, which has a low excitation energy with $m_{\Delta}-m_{N} \simeq 2 M_{\pi}$ and couples strongly to the $\pi N$ system [66]. To validate this hypothesis, we redo our analysis in the next section with the leading-order contributions of the $\Delta$-resonance being included explicitly.

\section{THE EXPLICIT INCLUSION OF THE LOWEST-ORDER $\Delta(1232)$ CONTRI- BUTIONS}

To quantify the importance of the $\Delta(1232)$ resonance for the description of $\pi N$ scattering at low energy, we include the leading-order $\Delta$ pole diagrams $\left(\delta^{1}\right)$ shown in Fig. 4 and repeat the fitting procedure described above. Note that the standard treatment of the $\Delta$ in the HB framework breaks down in the vicinity of the $\Delta$ pole. Therefore, we use the $\delta^{1}$ amplitudes calculated in the covariant framework based on the Lagrangian in [38] for all three counting schemes. It has to be emphasized that the inclusion of the $\Delta$ in such a way is a phenomenological procedure which is not based on a consistent power-counting such as the ones formulated in Refs. 67, 68]. A consistent inclusion of the $\Delta$ including loop contributions is deferred to a future publication. We use the same unitarization as in the previously discussed delta-less case (see Eq. (8)) and do not include explicitly the width of the $\Delta$ in our amplitudes. The only two new parameters which appear in the $\delta^{1}$-amplitudes are the mass of the $\Delta$, which is fixed to its Breit-Wigner value $m_{\Delta}=1.232 \mathrm{GeV}$, and the pion-nucleon- $\Delta$ coupling constant, which is fixed to its large $N_{C}$ value $g_{\pi N \Delta}=3 /(2 \sqrt{2}) g_{A, p h}=1.35$, where we have used $g_{A, p h}=1.27$. Notice that this value of $g_{\pi N \Delta}$ is close to the one extracted from the $\Delta$ width at leading order in the EFT expansion, see e.g. 69.

As in the case without $\Delta$, a representative fit to $\mathrm{d} \sigma / \mathrm{d} \Omega$ for the channel $\pi^{+} p \rightarrow \pi^{+} p$ at $T_{\pi}=43.3 \mathrm{MeV}$ is shown in Fig. 5. In Fig. 6, we show the reduced $\chi^{2}$ and $\bar{\chi}^{2}$ as a function of the maximal energy used in the fits. As expected and differently to the deltaless calculations, one observes in the all three counting schemes a fairly flat behavior of 
$\chi^{2} /$ dof as function of $T_{\pi}$ indicating that our estimation of the theoretical uncertainty is reasonable. Actually, $\chi^{2} /$ dof even tend to decrease with energy which may be viewed as an indication, that the actual breakdown scale $\Lambda_{b}$ of the resulting approach is somewhat higher than $600 \mathrm{MeV}$. Comparing the values of $\chi^{2} /$ dof in the delta-less and delta-full formulations, one furthermore realizes a significant improvement in the quality of the fits upon the explicit inclusion of the $\Delta$ isobar. A different treatment of the relativistic corrections does not have a significant impact on the quality of the fit except for the HB-NN results at order $Q^{2}+\delta^{1}$ which are considerably less accurate than those of the $\mathrm{HB}-\pi \mathrm{N}$ and covariant approaches at the same orders.

It is also comforting to see that the extracted LECs are now indeed rather stable with respect to increasing the energy range used in the fits contrary to the observed pattern in the delta-less case. The resulting values of the LECs at different chiral orders in the delta-full approach are collected in Table IV for all three counting schemes, while the corresponding correlation and covariance matrices are listed in Tables V] and VI. Here, we refrain from comparing the values of the LECs to the delta-less analyses available in the literature although such a comparison could, in principle, be done by explicitly taking into account the contributions of the $\Delta$ in the framework of resonance saturation. Remarkably, all extracted LECs including $e_{i}$ from the order- $Q^{4}$ pion-nucleon Lagrangian come out of a natural size for all considered counting schemes which is clearly not the case in the delta-less approach. Further, the differences between the values of the LECs extracted based on the different treatments of the relativistic corrections are much smaller as compared with the delta-less calculations. Also, the shifts in the LECs $c_{i}$ and $d_{i}$ when increasing the chiral order are now strongly reduced. All these findings provide a strong evidence that convergence of the EFT expansion for $\pi N$ scattering is considerably improved upon the explicit treatment of the $\Delta$-resonance.

Our predictions for the $S$-, $P$-, $D$ - and $F$-wave phase shifts are summarized in Figs. 17,22 , A comparison of the size of the uncertainty bands for $S$-, $P$ - and most of the $D$-waves with the ones of the delta-less approaches confirms the improved convergence of the delta-full theory. For the $S$ - and $P$-waves, one observes excellent agreement between the predicted phase shifts and the ones of Ref. [49] determined from the RS analyses. Furthermore, our predictions for the $D$-wave phase shifts agree rather well (within uncertainties) with the ones of the GWU-SAID PWA. For the $F$-waves, the treatment of the $1 / m_{N}$-corrections seems to play a more important role. In particular, for the $F_{35^{-}} F_{17}$ and $F_{37^{-}}$waves, one observes significant differences between the HB-NN results at order $Q^{4}+\epsilon^{1}$ and the ones based on the HB- $\pi$ N and covariant approaches, which appear to agree rather well with the GWU-SAID PWA. The employed approach to uncertainty quantification clearly underestimates the error for the $F$-waves in the HB-NN approach. On the other hand, it is comforting to see that a more complete treatment of the relativistic corrections leads to a better agreement with the GWU-SAID PWA. It is, however, difficult to make conclusive statements due to the absence of uncertainties in the GWU-SAID PWA.

For the subthreshold coefficients and the scattering length, the explicit inclusion of the $\Delta$ resonance does, with very few exceptions, noticeably improve both the order- $Q^{3}$ and order$Q^{4}$ results for all counting schemes. Further, our predictions within the covariant approach show clearly a better agreement with the values found in the RS analysis as compared to the predictions within the HB formulations. This holds true for both orders $Q^{3}+\delta^{1}$ and $Q^{4}+\delta^{1}$. We also observe that our theoretical uncertainty for the subthreshold coefficients is underestimated at order $Q^{4}+\delta^{1}$. It remains to be seen whether a more complete inclusion 
of the $\Delta$ resonance will allow for a better description of these quantities. It would also be interesting to study in detail the convergence of the $1 / m_{N}$ expansion for the subthreshold coefficients and to estimate the impact of other sources of uncertainties such as e.g. the one in the value of the pion-nucleon coupling constant and/or isospin-breaking effects which are not included in our analysis. Work along these lines is in progress.

\section{SUMMARY AND OUTLOOK}

The pertinent results of our paper can be summarized as follows:

- We have calculated the pion-nucleon scattering amplitude in the covariant formulation of $\chi \mathrm{PT}$ up to the order $Q^{4}$ within the modified EOMS scheme. We discuss in detail the renormalization and finite shifts of the various parameters in the effective Lagrangian.

- We have implemented the novel approach to estimate the theoretical uncertainty from the truncation of the chiral expansion formulated in Ref. [52] and performed fits to the available low-energy $\pi N$ scattering data using the HB-NN, HB- $\pi \mathrm{N}$ and the covariant versions of $\chi \mathrm{PT}$. The extracted values of the various LECs are found to be in a reasonably good agreement with the ones reported in the literature. All three approaches lead to the description of the experimental data of a similar quality which, however, exhibits a fairly small breakdown scale of the chiral expansion.

- By explicitly including the lowest-order contributions of the $\Delta$ isobar, we were able to unambiguously demonstrate that the slow convergence of the chiral expansion for $\pi \mathrm{N}$ scattering is related to the implicit treatment of the $\Delta$ resonance in the considered formulations of $\chi \mathrm{PT}$. After including the lowest-order contributions of the $\Delta$ in the scattering amplitude, the breakdown scale of the resulting EFT is found to be consistent with and probably even slightly larger than $\Lambda_{b} \sim 600 \mathrm{MeV}$. All LECs determined from the corresponding fits to the experimental data are found to be of a natural size at all orders and for all three counting schemes. Further, the extracted values of the LECs appear to be remarkably stable against increasing the maximal fitting energy, changing the order of the calculation and employing different counting rules for the $1 / m_{N}$-corrections. The predicted phase shifts in the $S$ - and $P$-waves at order $Q^{4}+\delta^{1}$ are in excellent agreement with the ones extracted in Ref. [49] within the RS analyses of $\pi N$ scattering; the predictions for the $D$ - and $F$-waves are found to agree reasonably well with the GWU-SAID PWA. We also compare our predictions for the subthreshold coefficients and the scattering lengths with their empirical values.

The results of our study provide an important step towards performing a combined analysis of the $\pi N \rightarrow \pi N$ and $\pi N \rightarrow \pi \pi N$ reactions, which is expected to result in an even more reliable determination of the various LECs. Given that one has to use experimental data for the second reaction, see Ref. [38 for a recent study along this line, it would be inappropriate to employ empirical phase shift analyses for the first one. The results of our work thus pave the way for a unified treatment of both reactions with regard to the available experimental information. Moreover, the inclusion of the theoretical uncertainty when performing the fits as implemented in our work is shown to stabilize the results against the variation of the maximal fitting energy (provided the effects of the $\Delta$ isobar are explicitly taken into account) which is a necessary prerequisite for carrying out a combined analysis of 
the $\pi N \rightarrow \pi N$ and $\pi N \rightarrow \pi \pi N$ processes. Apart from extending the calculations presented here to the single pion production reaction, it would also be interesting to directly confront the $\chi \mathrm{PT}$ results for the phase shifts with their recent determination in the framework of the Roy-Steiner equation [49] and to perform a more complete and consistent treatment of the delta contributions. Work along these lines is in progress.

\section{Acknowledgments}

We would like to thank Jambul Gegelia for helpful discussions and useful comments on the EOMS scheme and De-Liang Yao for cross checking all amplitudes. One of the authors (DS) is grateful to the staff at the Institute for Nuclear Theory at the University of Washington, Seattle, where a part of this work has been done. This work was supported by the DFG (SFB/TR 16, "Subnuclear Structure of Matter"), the ERC project 259218 NUCLEAREFT the Ruhr University Research School PLUS, funded by Germany's Excellence Initiative [DFG GSC 98/3] and by the Chinese Academy of Sciences (CAS) President's International Fellowship Initiative (PIFI) (Grant No. 2015VMA076). 


\section{Appendix A: Renormalization Rules}

In this appendix, the formulae related to the renormalization of the amplitudes are given. The notation for the integrals is the following

$$
\begin{aligned}
A_{0}\left(m_{0}^{2}\right) & =\frac{1}{\mathrm{i}} \int \frac{\mathrm{d}^{d} l}{(2 \pi)^{d}} \frac{1}{l^{2}-m_{0}^{2}}, \\
B_{0}\left(p^{2}, m_{0}^{2}, m_{1}^{2}\right) & =\frac{1}{\mathrm{i}} \int \frac{\mathrm{d}^{d} l}{(2 \pi)^{d}} \frac{1}{\left(l^{2}-m_{0}^{2}\right)\left((l+p)^{2}-m_{1}^{2}\right)}, \\
J_{0}(\omega) & =\frac{1}{\mathrm{i}} \int \frac{\mathrm{d}^{d} l}{(2 \pi)^{d}} \frac{1}{\left(l^{2}-M_{\pi}^{2}\right)(\omega+v \cdot l)} \\
C_{0}\left(p_{1}^{2},\left(p_{1}-p_{2}\right)^{2}, p_{2}^{2}, m_{0}^{2}, m_{1}^{2}, m_{2}^{2}\right) & =\frac{1}{\mathrm{i}} \int \frac{\mathrm{d}^{d} l}{(2 \pi)^{d}} \frac{1}{\left(l^{2}-m_{0}^{2}\right)\left(\left(l+p_{1}\right)^{2}-m_{1}^{2}\right)\left(\left(l+p_{2}\right)^{2}-m_{2}^{2}\right)}
\end{aligned}
$$

where the $+\mathrm{i} \epsilon$ prescription was suppressed.

\section{Mesonic Sector}

The renormalization rules for the pion mass, Z-factor and decay constant read

$$
\begin{aligned}
M^{2} & =M_{\pi}^{2}+\delta M^{(4)} \\
\delta M^{(4)} & =-\frac{2 l_{3} M_{\pi}^{4}}{F_{\pi}^{2}}+\frac{M_{\pi}^{2} A_{0}\left(M_{\pi}^{2}\right)}{2 F_{\pi}^{2}}, \\
Z_{\pi} & =1+\delta Z_{\pi}^{(4)} \\
\delta Z_{\pi}^{(4)} & =-\frac{2 l_{4} M_{\pi}^{2}}{F_{\pi}^{2}}-\frac{(-1+10 \alpha) A_{0}\left(M_{\pi}^{2}\right)}{F_{\pi}^{2}}, \\
F & =F_{\pi}+\delta F_{\pi}^{(4)} \\
\delta F_{\pi}^{(4)} & =-\frac{l_{4} M_{\pi}^{2}}{F_{\pi}}-\frac{A_{0}\left(M_{\pi}^{2}\right)}{F_{\pi}} .
\end{aligned}
$$

\section{Baryonic sector}

In the baryonic sector one has to differentiate between the covariant and heavy baryon approaches. The self-energy diagrams necessary for mass renormalization are shown in Fig. 23. The axial coupling constant was renormalized at the pion-nucleon vertex and the contributing diagrams are shown in Fig. 24. 


\section{a. Covariant chiral perturbation theory}

In covariant $\chi \mathrm{PT}$, the renormalization rule for the nucleon mass reads

$$
\begin{aligned}
m & =m_{N}+\delta m^{(2)}+\delta m^{(3)}+\delta m^{(4)} \\
\delta m^{(2)} & =4 c_{1} M_{\pi}^{2}, \\
\delta m^{(3)} & =-\frac{3 g_{A}^{2} m_{N} A_{0}\left(m_{N}^{2}\right)}{2 F_{\pi}^{2}}-\frac{3 g_{A}^{2} M_{\pi}^{2} m_{N} B_{0}\left(m_{N}^{2}, M_{\pi}^{2}, m_{N}^{2}\right)}{2 F_{\pi}^{2}}, \\
\delta m^{(4)} & =M_{\pi}^{4}\left(2 e_{115}+2 e_{116}+16 e_{38}-\frac{8 c_{1} l_{3}}{F_{\pi}^{2}}-\frac{3 c_{2}}{128 F_{\pi}^{2} \pi^{2}}\right) \\
& +\frac{\left(32 c_{1}-3\left(c_{2}+4 c_{3}\right)\right) M_{\pi}^{2} A_{0}\left(M_{\pi}^{2}\right)}{4 F_{\pi}^{2}}
\end{aligned}
$$

whereas the expression for the Z-Factor is given by

$$
\begin{aligned}
Z_{N} & =1+\delta Z_{N}^{(3)}+\delta Z_{N}^{(4)} \\
\delta Z_{N}^{(3)} & =\frac{3 g_{A}^{2} M_{\pi}^{2} m_{N}^{2}}{16 F_{\pi}^{2}\left(M_{\pi}^{2}-4 m_{N}^{2}\right) \pi^{2}}+\frac{3 g_{A}^{2}\left(5 M_{\pi}^{2}-12 m_{N}^{2}\right) A_{0}\left(M_{\pi}^{2}\right)}{4 F_{\pi}^{2}\left(M_{\pi}^{2}-4 m_{N}^{2}\right)} \\
& -\frac{3 g_{A}^{2} M_{\pi}^{2} A_{0}\left(m_{N}^{2}\right)}{F_{\pi}^{2}\left(M_{\pi}^{2}-4 m_{N}^{2}\right)}-\frac{3 g_{A}^{2} M_{\pi}^{2}\left(M_{\pi}^{2}-3 m_{N}^{2}\right) B_{0}\left(m_{N}^{2}, M_{\pi}^{2}, m_{N}^{2}\right)}{F_{\pi}^{2}\left(M_{\pi}^{2}-4 m_{N}^{2}\right)}, \\
\delta Z_{N}^{(4)} & =\frac{3 c_{2} M_{\pi}^{4}}{64 F_{\pi}^{2} m_{N} \pi^{2}}+\frac{3 c_{2} M_{\pi}^{2} A_{0}\left(M_{\pi}^{2}\right)}{2 F_{\pi}^{2} m_{N}} .
\end{aligned}
$$

The effective axial coupling constant is renormalized via

$$
\begin{aligned}
g & =g_{A}+\delta g^{(3)}+\delta g^{(4)} \\
\delta g^{(3)} & =-\frac{M_{\pi}^{2}\left(3 g_{A}^{3} m_{N}^{2}+32 F_{\pi}^{2}\left(2 d_{16}-d_{18}\right)\left(M_{\pi}^{2}-4 m_{N}^{2}\right) \pi^{2}\right)}{16 F_{\pi}^{2}\left(M_{\pi}^{2}-4 m_{N}^{2}\right) \pi^{2}} \\
& -\frac{g_{A}\left(\left(1+4 g_{A}^{2}\right) M_{\pi}^{2}-2\left(2+5 g_{A}^{2}\right) m_{N}^{2}\right) A_{0}\left(M_{\pi}^{2}\right)}{F_{\pi}^{2}\left(M_{\pi}^{2}-4 m_{N}^{2}\right)} \\
& +\frac{g_{A}\left(\left(2+3 g_{A}^{2}\right) M_{\pi}^{2}-8 m_{N}^{2}\right) A_{0}\left(m_{N}^{2}\right)}{F_{\pi}^{2}\left(M_{\pi}^{2}-4 m_{N}^{2}\right)}-\frac{g_{A}^{3} m_{N}^{2} B_{0}\left(M_{\pi}^{2}, m_{N}^{2}, m_{N}^{2}\right)}{F_{\pi}^{2}} \\
& +\frac{g_{A} M_{\pi}^{2}\left(\left(2+3 g_{A}^{2}\right) M_{\pi}^{2}-\left(8+9 g_{A}^{2}\right) m_{N}^{2}\right) B_{0}\left(m_{N}^{2}, M_{\pi}^{2}, m_{N}^{2}\right)}{F_{\pi}^{2}\left(M_{\pi}^{2}-4 m_{N}^{2}\right)} \\
& -\frac{g_{A}^{3} M_{\pi}^{2} m_{N}^{2} C_{0}\left(m_{N}^{2}, M_{\pi}^{2}, m_{N}^{2}, M_{\pi}^{2}, m_{N}^{2}, m_{N}^{2}\right)}{F_{\pi}^{2}},
\end{aligned}
$$




$$
\begin{aligned}
& \delta g^{(4)}=-\frac{g_{A} c_{3}\left(-3 M_{\pi}^{4}+10 M_{\pi}^{2} m_{N}^{2}+8 m_{N}^{4}\right)}{144 F_{\pi}^{2} m_{N} \pi^{2}}-\frac{g_{A} c_{4}\left(-3 M_{\pi}^{4}+10 M_{\pi}^{2} m_{N}^{2}+8 m_{N}^{4}\right)}{144 F_{\pi}^{2} m_{N} \pi^{2}} \\
& -\frac{g_{A} c_{2}\left(-33 M_{\pi}^{6}+224 M_{\pi}^{4} m_{N}^{2}+64 M_{\pi}^{2} m_{N}^{4}+36 m_{N}^{6}\right)}{2304 F_{\pi}^{2} m_{N}^{3} \pi^{2}} \\
& +\left(\frac{2 g_{A} c_{3}\left(M_{\pi}^{4}-M_{\pi}^{2} m_{N}^{2}\right)}{3 F_{\pi}^{2} m_{N}^{3}}+\frac{2 g_{A} c_{4}\left(M_{\pi}^{4}-M_{\pi}^{2} m_{N}^{2}\right)}{3 F_{\pi}^{2} m_{N}^{3}}\right. \\
& \left.-\frac{g_{A} c_{2}\left(-8 M_{\pi}^{6}+5 M_{\pi}^{4} m_{N}^{2}+48 M_{\pi}^{2} m_{N}^{4}\right)}{24 F_{\pi}^{2} m_{N}^{5}}\right) A_{0}\left(M_{\pi}^{2}\right) \\
& +\left(\frac{4 g_{A} c_{1} M_{\pi}^{2}}{F_{\pi}^{2} m_{N}}-\frac{2 g_{A} c_{4}\left(M_{\pi}^{4}-10 m_{N}^{4}\right)}{3 F_{\pi}^{2} m_{N}^{3}}-\frac{2 g_{A} c_{3}\left(M_{\pi}^{4}+2 m_{N}^{4}\right)}{3 F_{\pi}^{2} m_{N}^{3}}\right. \\
& \left.-\frac{g_{A} c_{2}\left(2 M_{\pi}^{6}-3 M_{\pi}^{4} m_{N}^{2}+M_{\pi}^{2} m_{N}^{4}+3 m_{N}^{6}\right)}{6 F_{\pi}^{2} m_{N}^{5}}\right) A_{0}\left(m_{N}^{2}\right) \\
& +\left(\frac{2 g_{A} c_{4}\left(-M_{\pi}^{6}+2 M_{\pi}^{4} m_{N}^{2}+8 M_{\pi}^{2} m_{N}^{4}\right)}{3 F_{\pi}^{2} m_{N}^{3}}-\frac{g_{A} c_{2}\left(2 M_{\pi}^{8}-7 M_{\pi}^{6} m_{N}^{2}+8 M_{\pi}^{4} m_{N}^{4}\right)}{6 F_{\pi}^{2} m_{N}^{5}}\right. \\
& \left.+\frac{4 g_{A} c_{1} M_{\pi}^{4}}{F_{\pi}^{2} m_{N}}-\frac{2 g_{A} c_{3}\left(M_{\pi}^{6}-2 M_{\pi}^{4} m_{N}^{2}+4 M_{\pi}^{2} m_{N}^{4}\right)}{3 F_{\pi}^{2} m_{N}^{3}}\right) B_{0}\left(m_{N}^{2}, M_{\pi}^{2}, m_{N}^{2}\right) \text {. }
\end{aligned}
$$

\section{b. Heavy-baryon chiral perturbation theory}

The HB expression for the nucleon mass reads

$$
\begin{aligned}
m & =m_{N}+\delta m^{(2)}+\delta m^{(3)}+\delta m^{(4)} \\
\delta m^{(2)} & =4 c_{1} M_{\pi}^{2} \\
\delta m^{(3)} & =-\frac{3 g_{A}^{2} M_{\pi}^{2} J_{0}(0)}{4 F_{\pi}^{2}}, \\
\delta m^{(4)} & =M_{\pi}^{4}\left(2 e_{115}+2 e_{116}+16 e_{38}-\frac{8 c_{1} l_{3}}{F_{\pi}^{2}}-\frac{3 c_{2}}{128 F_{\pi}^{2} \pi^{2}}+\frac{3 g_{A}^{2}}{64 F_{\pi}^{2} m_{N} \pi^{2}}\right) \\
& -M_{\pi}^{2}\left(\frac{-32 c_{1}+3\left(c_{2}+4 c_{3}\right)}{4 F_{\pi}^{2}}+\frac{3 g_{A}^{2}}{4 F_{\pi}^{2} m_{N}}\right) A_{0}\left(M_{\pi}^{2}\right),
\end{aligned}
$$

whereas the Z-Factor is given by

$$
\begin{aligned}
Z_{N} & =1+\delta Z_{N}^{(3)}+\delta Z_{N}^{(4)} \\
\delta Z_{N}^{(3)} & =-\frac{3 g_{A}^{2} M_{\pi}^{2}}{32 F_{\pi}^{2} \pi^{2}}+\frac{9 g_{A}^{2} A_{0}\left(M_{\pi}^{2}\right)}{4 F_{\pi}^{2}}, \\
\delta Z_{N}^{(4)} & =-\frac{9 g_{A}^{2} M_{\pi}^{2} J_{0}(0)}{8 F_{\pi}^{2} m_{N}} .
\end{aligned}
$$

The effective axial coupling constant in the HB approach is given by

$$
\begin{aligned}
g & =g_{A}+\delta g^{(3)}+\delta g^{(4)} \\
\delta g^{(3)} & =M_{\pi}^{2}\left(-4 d_{16}+2 d_{18}+\frac{g_{A}^{3}}{16 F_{\pi}^{2} \pi^{2}}\right)-\frac{\left(g_{A}+2 g_{A}^{3}\right) A_{0}\left(M_{\pi}^{2}\right)}{F_{\pi}^{2}}, \\
\delta g^{(4)} & =M_{\pi}^{2}\left(-\frac{4 g_{A}\left(c_{3}-2 c_{4}\right)}{3 F_{\pi}^{2}}+\frac{g_{A}+g_{A}^{3}}{F_{\pi}^{2} m_{N}}\right) J_{0}(0) .
\end{aligned}
$$




\section{Appendix B: Renormalization of LECs}

\section{Mesonic Sector}

The $\beta$-functions in the mesonic sector read

$$
\beta_{l_{1}}=\frac{1}{3}, \quad \beta_{l_{2}}=\frac{2}{3}, \quad \beta_{l_{3}}=-\frac{1}{2}, \quad \beta_{l_{4}}=2 .
$$

\section{Baryonic Sector}

In the baryonic sector we have to differentiate between the EOMS and HB renormalization rules.

a. Covariant chiral perturbation theory

In EOMS scheme, the $\beta$-functions of the $c_{i}$ read at order $Q^{3}$

$$
\begin{aligned}
& \beta_{c_{1}}^{(3)}=-\frac{3 g_{A}^{2} m_{N}}{4}, \\
& \beta_{c_{2}}^{(3)}=\left(-1+g_{A}^{2}\right)^{2} m_{N}, \\
& \beta_{c_{3}}^{(3)}=\frac{1}{2}\left(1-6 g_{A}^{2}+g_{A}^{4}\right) m_{N}, \\
& \beta_{c_{4}}^{(3)}=\frac{1}{2}\left(-1-2 g_{A}^{2}+3 g_{A}^{4}\right) m_{N},
\end{aligned}
$$

while the contributions at order $Q^{4}$ have the form

$$
\begin{aligned}
& \beta_{c_{1}}^{(4)}=9 g_{A}^{2} c_{1} m_{N}^{2} \\
& \beta_{c_{2}}^{(4)}=\frac{1}{3}\left(-4 c_{4}+g_{A}^{2}\left(3 c_{2}+8 c_{3}+4 c_{4}\right)\right) m_{N}^{2}, \\
& \beta_{c_{3}}^{(4)}=\frac{1}{6}\left(g_{A}^{2}\left(21 c_{2}+54 c_{3}-52 c_{4}\right)+20 c_{4}\right) m_{N}^{2}, \\
& \beta_{c_{4}}^{(4)}=\frac{1}{6}\left(3 c_{2}+8 c_{3}-20 c_{4}-g_{A}^{2}\left(15 c_{2}+24 c_{3}+2 c_{4}\right)\right) m_{N}^{2} .
\end{aligned}
$$

The corresponding finite shifts are given by

$$
\begin{aligned}
\delta \bar{c}_{1, f}^{(3)} & =\frac{3 g_{A}^{2} m_{N}}{128 \pi^{2}}, \\
\delta \bar{c}_{2, f}^{(3)} & =-\frac{\left(2+g_{A}^{4}\right) m_{N}}{32 \pi^{2}} \\
\delta \bar{c}_{3, f}^{(3)} & =\frac{9 g_{A}^{4} m_{N}}{64 \pi^{2}}, \\
\delta \bar{c}_{4, f}^{(3)} & =-\frac{g_{A}^{2}\left(5+g_{A}^{2}\right) m_{N}}{64 \pi^{2}}
\end{aligned}
$$


at order $Q^{3}$ and

$$
\begin{aligned}
\delta \bar{c}_{1, f}^{(4)} & =\frac{3 g_{A}^{2} c_{1} m_{N}^{2}}{16 \pi^{2}}, \\
\delta \bar{c}_{2, f}^{(4)} & =\frac{\left(-2 c_{4}+g_{A}^{2}\left(9 c_{2}+16 c_{3}+14 c_{4}\right)\right) m_{N}^{2}}{144 \pi^{2}} \\
\delta \bar{c}_{3, f}^{(4)} & =\frac{\left(g_{A}^{2}\left(-9 c_{2}+216 c_{3}-272 c_{4}\right)+16 c_{4}\right) m_{N}^{2}}{1152 \pi^{2}}, \\
\delta \bar{c}_{4, f}^{(4)} & =\frac{\left(9\left(-1+g_{A}^{2}\right) c_{2}-8\left(4 c_{3}+\left(2+11 g_{A}^{2}\right) c_{4}\right)\right) m_{N}^{2}}{1152 \pi^{2}}
\end{aligned}
$$

at order $Q^{4}$, respectively. Similarly, for the LECs $d_{i}$, we obtain

$$
\begin{aligned}
\beta_{d_{1}}^{(3)}+\beta_{d_{2}}^{(3)} & =\frac{1}{24}\left(1-4 g_{A}^{2}+3 g_{A}^{4}\right), \\
\beta_{d_{3}}^{(3)} & =0 \\
\beta_{d_{4}}^{(3)} & =\frac{1}{8} g_{A}\left(-1+g_{A}^{2}\right)^{2}, \\
\beta_{d_{5}}^{(3)} & =\frac{1}{24}\left(1-g_{A}^{2}\right), \\
\beta_{d_{10}}^{(3)} & =\frac{1}{4} g_{A}\left(-1+g_{A}^{4}\right), \\
\beta_{d_{11}}^{(3)} & =-\frac{1}{4} g_{A}\left(3-4 g_{A}^{2}+g_{A}^{4}\right), \\
\beta_{d_{12}}^{(3)} & =\frac{1}{2} g_{A}\left(-1+g_{A}^{2}\right)^{2}, \\
\beta_{d_{13}}^{(3)} & =-\frac{1}{2} g_{A}\left(-1+g_{A}^{2}\right)^{2}, \\
\beta_{d_{14}}^{(3)}-\beta_{d_{15}}^{(3)} & =\frac{1}{2}\left(-1+g_{A}^{2}\right)^{2}, \\
\beta_{d_{16}}^{(3)} & =\frac{1}{2} g_{A}\left(-1+g_{A}^{2}\right) \\
\beta_{d_{18}}^{(3)} & =0
\end{aligned}
$$

and

$$
\begin{aligned}
\beta_{d_{1}}^{(4)}+\beta_{d_{2}}^{(4)} & =\frac{\left(\left(7+11 g_{A}^{2}\right) c_{2}-16\left(-1+g_{A}^{2}\right) c_{3}+2\left(-5+g_{A}^{2}\right) c_{4}\right) m_{N}}{12} \\
\beta_{d_{3}}^{(4)} & =-\frac{5\left(-1+g_{A}^{2}\right) c_{2} m_{N}}{3} \\
\beta_{d_{4}}^{(4)} & =\frac{g_{A}\left(\left(9-15 g_{A}^{2}\right) c_{2}+4\left(3 c_{3}+5 c_{4}\right)-4 g_{A}^{2}\left(3 c_{3}+14 c_{4}\right)\right) m_{N}}{24} \\
\beta_{d_{5}}^{(4)} & =\frac{\left(24\left(-3+2 g_{A}^{2}\right) c_{1}-3 c_{2}+8 c_{3}+4 c_{4}+2 g_{A}^{2}\left(-2 c_{3}+c_{4}\right)\right) m_{N}}{24} \\
\beta_{d_{10}}^{(4)} & =-\frac{g_{A}\left(\left(-71+63 g_{A}^{2}\right) c_{2}+64\left(-3+g_{A}^{2}\right) c_{3}+24\left(3+g_{A}^{2}\right) c_{4}\right) m_{N}}{24}
\end{aligned}
$$




$$
\begin{aligned}
\beta_{d_{11}}^{(4)} & =\frac{g_{A}\left(\left(-33+13 g_{A}^{2}\right) c_{2}-96 c_{3}+8\left(-17+11 g_{A}^{2}\right) c_{4}\right) m_{N}}{24}, \\
\beta_{d_{12}}^{(4)} & =\frac{g_{A}\left(c_{2}-13 g_{A}^{2} c_{2}-16\left(-3+g_{A}^{2}\right) c_{3}+112\left(-1+g_{A}^{2}\right) c_{4}\right) m_{N}}{24}, \\
\beta_{d_{13}}^{(4)} & =\frac{g_{A}\left(\left(11-15 g_{A}^{2}\right) c_{2}+16\left(\left(-3+g_{A}^{2}\right) c_{3}-7\left(-1+g_{A}^{2}\right) c_{4}\right)\right) m_{N}}{24}, \\
\beta_{d_{14}}^{(4)}-\beta_{d_{15}}^{(4)} & =\frac{\left(g_{A}^{2}\left(-13 c_{2}+8 c_{3}-12 c_{4}\right)+12 c_{4}\right) m_{N}}{6}, \\
\beta_{d_{16}}^{(4)} & =\frac{g_{A}\left(-24\left(-2+g_{A}^{2}\right) c_{1}+c_{2}+6\left(c_{3}-3 c_{4}\right)\right) m_{N}}{6}, \\
\beta_{d_{18}}^{(4)} & =\frac{g_{A}\left(24 c_{1}+c_{2}-4\left(c_{3}+c_{4}\right)\right) m_{N}}{6},
\end{aligned}
$$

while the finite shifts have the form

$$
\begin{aligned}
\delta \bar{d}_{1, f}^{(3)}+\delta \bar{d}_{2, f}^{(3)} & =-\frac{12+39 g_{A}^{2}+11 g_{A}^{4}}{768 \pi^{2}}, \\
\delta \bar{d}_{3, f}^{(3)} & =\frac{3+3 g_{A}^{2}+g_{A}^{4}}{96 \pi^{2}}, \\
\delta \bar{d}_{4, f}^{(3)} & =\frac{g_{A}\left(7+2 g_{A}^{2}+g_{A}^{4}\right)}{256 \pi^{2}}, \\
\delta \bar{d}_{5, f}^{(3)} & =\frac{g_{A}^{2}\left(9+g_{A}^{2}\right)}{512 \pi^{2}}, \\
\delta \bar{d}_{10, f}^{(3)} & =\frac{g_{A}\left(18+81 g_{A}^{2}+31 g_{A}^{4}\right)}{384 \pi^{2}}, \\
\delta \bar{d}_{11, f}^{(3)} & =-\frac{g_{A}^{3}\left(33+19 g_{A}^{2}\right)}{384 \pi^{2}}, \\
\delta \bar{d}_{12, f}^{(3)} & =-\frac{g_{A}\left(36+46 g_{A}^{2}+29 g_{A}^{4}\right)}{192 \pi^{2}} \\
\delta \bar{d}_{13, f}^{(3)} & =\frac{g_{A}\left(12+22 g_{A}^{2}+13 g_{A}^{4}\right)}{192 \pi^{2}}, \\
\delta \bar{d}_{14, f}^{(3)}-\delta \bar{d}_{15, f}^{(3)} & =\frac{g_{A}^{4}}{192 \pi^{2}}, \\
\delta \bar{d}_{16, f}^{(3)} & =\frac{g_{A}+g_{A}^{3}}{32 \pi^{2}}, \\
\delta \bar{d}_{18, f}^{(3)} & =\frac{g_{A}^{3}}{192 \pi^{2}}
\end{aligned}
$$

and

$$
\begin{aligned}
\delta \bar{d}_{1, f}^{(4)}+\delta \bar{d}_{2, f}^{(4)} & =-\frac{\left(\left(4+8 g_{A}^{2}\right) c_{2}+\left(10-22 g_{A}^{2}\right) c_{3}+\left(5+38 g_{A}^{2}\right) c_{4}\right) m_{N}}{576 \pi^{2}} \\
\delta \bar{d}_{3, f}^{(4)} & =\frac{\left(\left(-34+4 g_{A}^{2}\right) c_{2}+3\left(-10 c_{3}+c_{4}+5 g_{A}^{2} c_{4}\right)\right) m_{N}}{288 \pi^{2}} \\
\delta \bar{d}_{4, f}^{(4)} & =\frac{g_{A}\left(6 c_{3}+184 c_{4}+g_{A}^{2}\left(-9\left(c_{2}+2 c_{3}\right)+2 c_{4}\right)\right) m_{N}}{1152 \pi^{2}} \\
\delta \bar{d}_{5, f}^{(4)} & =\frac{\left(72\left(2+g_{A}^{2}\right) c_{1}-2\left(1+19 g_{A}^{2}\right) c_{3}+\left(-1+10 g_{A}^{2}\right) c_{4}\right) m_{N}}{1152 \pi^{2}}
\end{aligned}
$$




$$
\begin{aligned}
\delta \bar{d}_{10, f}^{(4)} & =-\frac{g_{A}\left(\left(41+195 g_{A}^{2}\right) c_{2}+704 g_{A}^{2}\left(c_{3}-3 c_{4}\right)+48\left(10 c_{3}-3 c_{4}\right)\right) m_{N}}{4608 \pi^{2}} \\
\delta \bar{d}_{11, f}^{(4)} & =\frac{g_{A}\left(87 c_{2}+5 g_{A}^{2} c_{2}+1056 c_{3}-16\left(5+64 g_{A}^{2}\right) c_{4}\right) m_{N}}{4608 \pi^{2}}, \\
\delta \bar{d}_{12, f}^{(4)} & =-\frac{g_{A}\left(\left(-329+341 g_{A}^{2}\right) c_{2}+32\left(-33+4 g_{A}^{2}\right) c_{3}+64\left(5+7 g_{A}^{2}\right) c_{4}\right) m_{N}}{4608 \pi^{2}} \\
\delta \bar{d}_{13, f}^{(4)} & =\frac{g_{A}\left(\left(-485+33 g_{A}^{2}\right) c_{2}+32\left(-33 c_{3}+4 c_{4}\right)+64 g_{A}^{2}\left(5 c_{3}+7 c_{4}\right)\right) m_{N}}{4608 \pi^{2}} \\
\delta \bar{d}_{14, f}^{(4)}-\delta \bar{d}_{15, f}^{(4)} & =\frac{\left(24 c_{4}+g_{A}^{2}\left(67 c_{2}-56 c_{3}+96 c_{4}\right)\right) m_{N}}{1152 \pi^{2}}, \\
\delta \bar{d}_{16, f}^{(4)} & =-\frac{g_{A}\left(72\left(-1+g_{A}^{2}\right) c_{1}+c_{2}+18\left(c_{3}-c_{4}\right)\right) m_{N}}{288 \pi^{2}} \\
\delta \bar{d}_{18, f}^{(4)} & =\frac{g_{A}\left(c_{2}-c_{3}-c_{4}\right) m_{N}}{144 \pi^{2}} .
\end{aligned}
$$

Finally, we also list the $\beta$-functions and the finite shifts for the LECs $e_{i}$ :

$$
\begin{aligned}
\beta_{e_{10}}^{(4)}= & \frac{1}{192} g_{A}\left(\left(101-41 g_{A}^{2}\right) c_{2}+16\left(\left(-3+g_{A}^{2}\right) c_{3}-7\left(-1+g_{A}^{2}\right) c_{4}\right)\right) \\
& \quad-\frac{g_{A}\left(-1+g_{A}^{2}\right)^{2}}{16 m_{N}}, \\
\beta_{e_{11}}^{(4)}= & -\frac{1}{24} g_{A}\left(\left(-35+39 g_{A}^{2}\right) c_{2}+22\left(-1+g_{A}^{2}\right) c_{3}+\left(-29+83 g_{A}^{2}\right) c_{4}\right) \\
& \quad+\frac{g_{A}\left(19-40 g_{A}^{2}+21 g_{A}^{4}\right)}{96 m_{N}}, \\
\beta_{e_{12}}^{(4)}= & \frac{1}{24} g_{A}\left(\left(-25+29 g_{A}^{2}\right) c_{2}+22\left(-1+g_{A}^{2}\right) c_{3}+\left(-29+83 g_{A}^{2}\right) c_{4}\right) \\
& \quad-\frac{g_{A}\left(19-40 g_{A}^{2}+21 g_{A}^{4}\right)}{96 m_{N}}, \\
\beta_{e_{13}}^{(4)}= & \frac{1}{12} g_{A}\left(-1+g_{A}^{2}\right) c_{2}, \\
\beta_{e_{14}}^{(4)}= & \frac{1}{96}\left(-\left(8+25 g_{A}^{2}\right) c_{2}+4\left(2\left(-6+g_{A}^{2}\right) c_{3}+5\left(-1+g_{A}^{2}\right) c_{4}\right)\right) \\
& \quad+\frac{\left(-1+g_{A}^{2}\right)^{2}}{32 m_{N}}, \\
\beta_{e_{18}}^{(4)}= & \frac{1}{4}\left(c_{2}-g_{A}^{2} c_{2}\right), \\
\beta_{e_{15}}^{(4)}= & \frac{g_{A}^{2} c_{2}}{6}, \\
\beta_{e_{16}}^{(4)}= & 0, \\
\beta_{17}^{(4)}= & \frac{1}{48}\left(\left(-1+15 g_{A}^{2}\right) c_{2}+2\left(1-7 g_{A}^{2}\right) c_{4}\right)+\frac{1-4 g_{A}^{2}+3 g_{A}^{4}}{96 m_{N}}, \\
&
\end{aligned}
$$




$$
\begin{aligned}
2 \beta_{e_{19}}^{(4)}-\beta_{e_{22}}^{(4)}-\beta_{e_{36}}^{(4)}= & 2 c_{1}+\frac{1}{24}\left(-5+36 g_{A}^{2}\right) c_{2}+\frac{3 c_{3}}{4}+\frac{5 g_{A}^{2} c_{3}}{12}+c_{4}-g_{A}^{2} c_{4} \\
& -\frac{\left(-1+g_{A}^{2}\right)^{2}}{8 m_{N}}, \\
\beta_{e_{2}}^{(4)}+\beta_{e_{35}}^{(4)}= & -\frac{1}{12}\left(-6+g_{A}^{2}\right) c_{2}, \\
2 \beta_{e_{21}}^{(4)}-\beta_{e_{37}}^{(4)}= & \frac{1}{24}\left(-24 c_{1}+c_{2}-4 g_{A}^{2} c_{2}+4\left(4 c_{3}-3 g_{A}^{2} c_{3}+7 g_{A}^{2} c_{4}\right)\right) \\
& +\frac{1-g_{A}^{2}}{24 m_{N}}, \\
\beta_{e_{22}}^{(4)}-4 \beta_{e_{38}}^{(4)}= & \frac{1}{48}\left(-72\left(2+g_{A}^{2}\right) c_{1}+12 c_{2}-39 g_{A}^{2} c_{2}+36 c_{3}+8 g_{A}^{2} c_{3}-4 c_{4}+4 g_{A}^{2} c_{4}\right) \\
& +\frac{\left(-1+g_{A}^{2}\right)^{2}}{16 m_{N}}, \\
\beta_{e_{34}}^{(4)}= & \frac{1}{48} g_{A}\left(-48 c_{1}+\left(-25+49 g_{A}^{2}\right) c_{2}+4\left(-7 c_{3}+9 g_{A}^{2} c_{3}-17 c_{4}+43 g_{A}^{2} c_{4}\right)\right) \\
& -\frac{g_{A}\left(7-16 g_{A}^{2}+9 g_{A}^{4}\right)}{48 m_{N}}
\end{aligned}
$$

and the finite pieces

$$
\begin{aligned}
\delta \bar{e}_{10, f}^{(4)}=- & \frac{g_{A}\left(\left(503+493 g_{A}^{2}\right) c_{2}-32\left(\left(9+2 g_{A}^{2}\right) c_{3}-8\left(13+6 g_{A}^{2}\right) c_{4}\right)\right)}{36864 \pi^{2}} \\
& -\frac{g_{A}\left(30+38 g_{A}^{2}+17 g_{A}^{4}\right)}{1536 m_{N} \pi^{2}}, \\
\delta \bar{e}_{11, f}^{(4)}=- & \frac{g_{A}\left(\left(69+70 g_{A}^{2}\right) c_{2}-4\left(-5+8 g_{A}^{2}\right) c_{3}+\left(-244+233 g_{A}^{2}\right) c_{4}\right)}{2304 \pi^{2}} \\
& +\frac{g_{A}\left(46-47 g_{A}^{2}+87 g_{A}^{4}\right)}{3072 m_{N} \pi^{2}}, \\
\delta \bar{e}_{12, f}^{(4)}= & \frac{g_{A}\left(\left(29+50 g_{A}^{2}\right) c_{2}+8\left(1+g_{A}^{2}\right) c_{3}+\left(38+375 g_{A}^{2}\right) c_{4}\right)}{2304 \pi^{2}} \\
& +\frac{g_{A}\left(162+435 g_{A}^{2}+13 g_{A}^{4}\right)}{3072 m_{N} \pi^{2}}, \\
\delta \bar{e}_{13, f}^{(4)}= & \frac{g_{A}\left(\left(40-7 g_{A}^{2}\right) c_{2}+\left(65+2 g_{A}^{2}\right) c_{3}-19\left(c_{4}+4 g_{A}^{2} c_{4}\right)\right)}{576 \pi^{2}} \\
\delta \bar{e}_{14, f}^{(4)}= & \frac{-40 c_{4}+g_{A}^{2}\left(-53 c_{2}+184 c_{3}+160 c_{4}\right)}{18432 \pi^{2}}-\frac{6-12 g_{A}^{2}+11 g_{A}^{4}}{1536 m_{N} \pi^{2}} \\
\delta \bar{e}_{15, f}^{(4)}= & \frac{12 c_{4}+g_{A}^{2}\left(7 c_{2}-2\left(4 c_{3}+c_{4}\right)\right)}{576 \pi^{2}}+\frac{21+22 g_{A}^{2}+7 g_{A}^{4}}{768 m_{N} \pi^{2}}, \\
\delta \bar{e}_{16, f}^{(4)}= & \frac{3 c_{4}+g_{A}^{2}\left(-3 c_{2}-2 c_{3}+c_{4}\right)}{288 \pi^{2}}-\frac{6+12 g_{A}^{2}+5 g_{A}^{4}}{256 m_{N} \pi^{2}},
\end{aligned}
$$




$$
\begin{aligned}
& \delta \bar{e}_{17, f}^{(4)}=\frac{\left(4+6 g_{A}^{2}\right) c_{2}+6\left(1+g_{A}^{2}\right) c_{3}+\left(3+20 g_{A}^{2}\right) c_{4}}{2304 \pi^{2}} \\
& +\frac{g_{A}^{2}\left(11+13 g_{A}^{2}\right)}{3072 m_{N} \pi^{2}} \\
& \delta \bar{e}_{18, f}^{(4)}=-\frac{6\left(1+g_{A}^{2}\right) c_{2}+\left(-6+4 g_{A}^{2}\right) c_{3}+\left(15+19 g_{A}^{2}\right) c_{4}}{1152 \pi^{2}} \\
& -\frac{3+12 g_{A}^{2}+8 g_{A}^{4}}{768 m_{N} \pi^{2}} \\
& 2 \delta \bar{e}_{19, f}^{(4)}-\delta \bar{e}_{22, f}^{(4)}-\delta \bar{e}_{36, f}^{(4)}=-\frac{72 c_{4}+g_{A}^{2}\left(144 c_{1}-69 c_{2}+28 c_{3}+96 c_{4}\right)}{4608 \pi^{2}}+\frac{3-7 g_{A}^{2}+27 g_{A}^{4}}{768 m_{N} \pi^{2}} \\
& \delta \bar{e}_{20, f}^{(4)}+\delta \bar{e}_{35, f}^{(4)}=\frac{g_{A}^{2}\left(72 c_{1}-5 c_{2}+72 c_{3}-24 c_{4}\right)-48 c_{4}}{2304 \pi^{2}}+\frac{-12+40 g_{A}^{2}+19 g_{A}^{4}}{1536 m_{N} \pi^{2}} \\
& 2 \delta \bar{e}_{21, f}^{(4)}-\delta \bar{e}_{37, f}^{(4)}=\frac{-4 c_{2}-34 c_{3}+19 c_{4}+g_{A}^{2}\left(72 c_{1}+12 c_{2}-6 c_{3}+35 c_{4}\right)}{1152 \pi^{2}} \\
& +\frac{6+25 g_{A}^{2}+3 g_{A}^{4}}{1536 m_{N} \pi^{2}} \\
& \delta \bar{e}_{22, f}^{(4)}-4 \delta \bar{e}_{38, f}^{(4)}=-\frac{8 c_{4}+g_{A}^{2}\left(720 c_{1}+153 c_{2}-136 c_{3}+16 c_{4}\right)}{9216 \pi^{2}} \\
& -\frac{2+12 g_{A}^{2}+3 g_{A}^{4}}{512 m_{N} \pi^{2}} \\
& \delta \bar{e}_{34, f}^{(4)}=\frac{g_{A}\left(576 c_{1}+3\left(4+11 g_{A}^{2}\right) c_{2}-110 c_{3}+42 g_{A}^{2} c_{3}-101 c_{4}+137 g_{A}^{2} c_{4}\right)}{2304 \pi^{2}} \\
& -\frac{g_{A}\left(17-11 g_{A}^{2}+42 g_{A}^{4}\right)}{1536 m_{N} \pi^{2}} .
\end{aligned}
$$

\section{b. Heavy-baryon chiral perturbation theory}

In the HB formulation, the employed $\beta$-functions at order $Q^{3}$ read

$$
\begin{array}{rlrl}
\beta_{d_{1}} & =-\frac{g_{A}^{4}}{6}, & \beta_{d_{2}} & =\frac{1}{12}\left(-1-5 g_{A}^{2}\right), \\
\beta_{d_{3}} & =\frac{1}{6}\left(3+g_{A}^{4}\right), & \beta_{d_{4}} & =0, \\
\beta_{d_{5}} & =\frac{1}{24}\left(1+5 g_{A}^{2}\right), & \beta_{d_{10}} & =\frac{1}{2}\left(g_{A}+5 g_{A}^{3}+4 g_{A}^{5}\right), \\
\beta_{d_{11}} & =\frac{1}{6}\left(3 g_{A}-9 g_{A}^{3}-4 g_{A}^{5}\right), & \beta_{d_{12}} & =-g_{A}\left(2+g_{A}^{2}+2 g_{A}^{4}\right), \\
\beta_{d_{13}} & =g_{A}^{3}+\frac{2 g_{A}^{5}}{3}, & \beta_{d_{14}} & =\frac{g_{A}^{4}}{3}, \\
\beta_{d_{15}} & =0, & \beta_{d_{16}} & =\frac{g_{A}}{2}+g_{A}^{3}, \\
\beta_{d_{18}} & =0 . &
\end{array}
$$


For the $\beta$-functions at order $Q^{4}$ the following results are obtained:

$$
\begin{aligned}
& \beta_{e_{10}}=-\frac{1}{6} g_{A}\left(3+8 g_{A}^{2}\right) c_{4}-\frac{g_{A}\left(3+19 g_{A}^{2}+13 g_{A}^{4}\right)}{24 m_{N}}, \\
& \beta_{e_{11}}=-\frac{g_{A} c_{4}}{3}+\frac{g_{A}\left(-7+35 g_{A}^{2}+12 g_{A}^{4}\right)}{48 m_{N}}, \\
& \beta_{e_{12}}=\frac{4}{3} g_{A}\left(1+g_{A}^{2}\right) c_{4}+\frac{g_{A}\left(61+57 g_{A}^{2}+26 g_{A}^{4}\right)}{48 m_{N}}, \\
& \beta_{e_{13}}=-\frac{2}{3}\left(g_{A}+2 g_{A}^{3}\right) c_{4}-\frac{g_{A}\left(73+54 g_{A}^{2}+21 g_{A}^{4}\right)}{24 m_{N}}, \\
& \beta_{e_{14}}=\frac{1}{12}\left(-c_{2}-6 c_{3}\right)-\frac{g_{A}^{2}\left(3+g_{A}^{2}\right)}{12 m_{N}}, \\
& \beta_{e_{15}}=\frac{9+2 g_{A}^{2}+11 g_{A}^{4}}{24 m_{N}} \\
& \beta_{e_{16}}=\frac{-3-2 g_{A}^{2}-2 g_{A}^{4}}{4 m_{N}}, \\
& \beta_{e_{17}}=-\frac{c_{4}}{12}+\frac{-1+7 g_{A}^{2}+4 g_{A}^{4}}{48 m_{N}}, \\
& \beta_{e_{18}}=-\frac{2 g_{A}^{2} c_{4}}{3}-\frac{g_{A}^{2}\left(3+4 g_{A}^{2}\right)}{12 m_{N}}, \\
& 2 \beta_{e_{19}}-\beta_{e_{22}}-\beta_{e_{36}}=2 c_{1}-\frac{5 c_{2}}{24}+\frac{3 c_{3}}{4}+\frac{-1+g_{A}^{2}-6 g_{A}^{4}}{8 m_{N}}, \\
& \beta_{e_{20}}+\beta_{e_{35}}=\frac{c_{2}}{2}+\frac{6+16 g_{A}^{2}+15 g_{A}^{4}}{24 m_{N}}, \\
& 2 \beta_{e_{21}}-\beta_{e_{37}}=\frac{1}{3}\left(2+9 g_{A}^{2}\right) c_{4}+\frac{2+16 g_{A}^{2}+9 g_{A}^{4}}{12 m_{N}} \\
& \beta_{e_{22}}-4 \beta_{e_{38}}=\frac{1}{4}\left(-12 c_{1}+c_{2}+3 c_{3}\right), \\
& \beta_{e_{34}}=\frac{2 g_{A} c_{4}}{3}+\frac{g_{A}-7 g_{A}^{3}-6 g_{A}^{5}}{24 m_{N}} \text {. }
\end{aligned}
$$

[1] S. Weinberg, Physica A 96, 327 (1979).

[2] J. Gasser and H. Leutwyler, Annals Phys. 158, 142 (1984).

[3] J. Gasser and H. Leutwyler, Nucl. Phys. B 250, 465 (1985).

[4] J. Gasser, M. E. Sainio and A. Svarc, Nucl. Phys. B 307, 779 (1988).

[5] V. Bernard, N. Kaiser and U.-G. Meißner, Int. J. Mod. Phys. E 4, 193 (1995) [hep-ph/9501384].

[6] V. Bernard and U.-G. Meißner, Ann. Rev. Nucl. Part. Sci. 57, 33 (2007) hep-ph/0611231.

[7] V. Bernard, Prog. Part. Nucl. Phys. 60, 82 (2008) [arXiv:0706.0312 [hep-ph]].

[8] S. Weinberg, Phys. Lett. B 251, 288 (1990).

[9] C. Ordonez, L. Ray and U. van Kolck, Phys. Rev. C 53, 2086 (1996) hep-ph/9511380.

[10] E. Epelbaum, H. W. Hammer and U.-G. Meißner, Rev. Mod. Phys. 81, 1773 (2009) arXiv:0811.1338 [nucl-th]].

[11] R. Machleidt and D. R. Entem, Phys. Rept. 503, 1 (2011) arXiv:1105.2919 [nucl-th]]. 
[12] E. E. Jenkins and A. V. Manohar, Phys. Lett. B 255, 558 (1991).

[13] V. Bernard, N. Kaiser, J. Kambor and U.-G. Meißner, Nucl. Phys. B 388, 315 (1992).

[14] V. Bernard, N. Kaiser, U.-G. Meißner and A. Schmidt, Z. Phys. A 348 (1994) 317

[15] V. Bernard, N. Kaiser and U.-G. Meißner, Nucl. Phys. A 611, 429 (1996) hep-ph/9607428.

[16] T. Becher and H. Leutwyler, Eur. Phys. J. C 9, 643 (1999) hep-ph/9901384.

[17] P. J. Ellis and H. B. Tang, Phys. Rev. C 57, 3356 (1998) hep-ph/9709354.

[18] V. Bernard, T. R. Hemmert and U.-G. Meißner, Phys. Rev. D 67, 076008 (2003) hep$\mathrm{ph} / 0212033$.

[19] J. Gegelia and G. Japaridze, Phys. Rev. D 60, 114038 (1999) hep-ph/9908377].

[20] T. Fuchs, J. Gegelia, G. Japaridze and S. Scherer, Phys. Rev. D 68, 056005 (2003) hepph/0302117].

[21] M. Mojzis, Eur. Phys. J. C 2, 181 (1998) hep-ph/9704415.

[22] N. Fettes, U.-G. Meißner and S. Steininger, Nucl. Phys. A 640, 199 (1998) hep-ph/9803266].

[23] P. Buettiker and U.-G. Meißner, Nucl. Phys. A 668, 97 (2000) hep-ph/9908247).

[24] N. Fettes, U.-G. Meißner, M. Mojzis and S. Steininger, Annals Phys. 283, 273 (2000) [Annals Phys. 288, 249 (2001)] hep-ph/0001308.

[25] N. Fettes and U.-G. Meißner, Nucl. Phys. A 676, 311 (2000) hep-ph/0002162.

[26] T. Becher and H. Leutwyler, JHEP 0106, 017 (2001) hep-ph/0103263.

[27] M. Hoferichter, B. Kubis and U.-G. Meißner, Nucl. Phys. A 833, 18 (2010) arXiv:0909.4390 [hep-ph]].

[28] A. Gasparyan and M. F. M. Lutz, Nucl. Phys. A 848, 126 (2010) arXiv:1003.3426 [hep-ph]].

[29] J. M. Alarcon, J. Martin Camalich and J. A. Oller, Annals Phys. 336, 413 (2013) arXiv:1210.4450 [hep-ph]].

[30] Y. H. Chen, D. L. Yao and H. Q. Zheng, Phys. Rev. D 87, 054019 (2013) arXiv:1212.1893 [hep-ph]].

[31] J. Beringer, PiN Newslett. 7, 33 (1992).

[32] V. Bernard, N. Kaiser and U.-G. Meißner, Phys. Lett. B 332, 415 (1994) [Phys. Lett. B 338, 520 (1994)] [hep-ph/9404236].

[33] V. Bernard, N. Kaiser and U.-G. Meißner, Nucl. Phys. B 457, 147 (1995) hep-ph/9507418].

[34] T. S. Jensen and A. F. Miranda, Phys. Rev. C 55, 1039 (1997).

[35] N. Fettes, V. Bernard and U.-G. Meißner, Nucl. Phys. A 669, 269 (2000) hep-ph/9907276].

[36] V. Bernard, N. Kaiser and U.-G. Meißner, Nucl. Phys. A 619, 261 (1997) hep-ph/9703218.

[37] N. Mobed, J. Zhang and D. Singh, Phys. Rev. C 72, 045204 (2005).

[38] D. Siemens, V. Bernard, E. Epelbaum, H. Krebs and U.-G. Meißner, Phys. Rev. C 89, no. 6, 065211 (2014) arXiv:1403.2510 [nucl-th]].

[39] H. Krebs, A. Gasparyan and E. Epelbaum, Phys. Rev. C 85, 054006 (2012) arXiv:1203.0067 [nucl-th]].

[40] K. A. Wendt, B. D. Carlsson and A. Ekström, arXiv:1410.0646 [nucl-th].

[41] D. R. Entem, N. Kaiser, R. Machleidt and Y. Nosyk, Phys. Rev. C 91, no. 1, 014002 (2015) arXiv:1411.5335 [nucl-th]].

[42] D. R. Entem, N. Kaiser, R. Machleidt and Y. Nosyk, Phys. Rev. C 92, no. 6, 064001 (2015) arXiv:1505.03562 [nucl-th]].

[43] E. Epelbaum, H. Krebs and U.-G. Meißner, Phys. Rev. Lett. 115, no. 12, 122301 (2015) arXiv:1412.4623 [nucl-th]].

[44] M. C. M. Rentmeester, R. G. E. Timmermans, J. L. Friar and J. J. de Swart, Phys. Rev. Lett. 82, 4992 (1999) nucl-th/9901054. 
[45] M. C. Birse and J. A. McGovern, Phys. Rev. C 70, 054002 (2004) nucl-th/0307050].

[46] E. Epelbaum, arXiv:1510.07036 [nucl-th].

[47] R. Koch and E. Pietarinen, Nucl. Phys. A 336, 331 (1980).

[48] R. A. Arndt, W. J. Briscoe, I. I. Strakovsky and R. L. Workman, Phys. Rev. C 74, 045205 (2006) [nucl-th/0605082.

[49] M. Hoferichter, J. Ruiz de Elvira, B. Kubis and U.-G. Meißner, Phys. Rev. Lett. 115, 092301 (2015) arXiv:1506.04142 [hep-ph]].

[50] M. Hoferichter, J. Ruiz de Elvira, B. Kubis and U.-G. Meißner, Phys. Rev. Lett. 115, no. 19, 192301 (2015) arXiv:1507.07552 [nucl-th]].

[51] M. Hoferichter, J. R. de Elvira, B. Kubis and U.-G. Meißner, accepted for publication in Phys. Rep. [arXiv:1510.06039 [hep-ph]].

[52] E. Epelbaum, H. Krebs and U.-G. Meißner, Eur. Phys. J. A 51, no. 5, 53 (2015) arXiv:1412.0142 [nucl-th]].

[53] S. Binder et al., arXiv:1505.07218 [nucl-th].

[54] G. Höhler and H. Schopper, Berlin, Germany: Springer (1983) 601 P. (Landolt-boernstein. New Series, I/9B2)

[55] B. Tromborg, S. Waldenstrom and I. Overbo, Phys. Rev. D 15, 725 (1977).

[56] V. Baru, C. Hanhart, M. Hoferichter, B. Kubis, A. Nogga and D. R. Phillips, Phys. Lett. B 694, 473 (2011) arXiv:1003.4444 [nucl-th]].

[57] U.-G. Meißner, hep-ph/9805440.

[58] J. Gasser, M. A. Ivanov, E. Lipartia, M. Mojzis and A. Rusetsky, Eur. Phys. J. C 26, 13 (2002) hep-ph/0206068.

[59] M. Hoferichter, B. Kubis and U.-G. Meißner, Phys. Lett. B 678, 65 (2009) arXiv:0903.3890 [hep-ph]].

[60] K. A. Olive et al. [Particle Data Group Collaboration], Chin. Phys. C 38, 090001 (2014).

[61] R. L. Workman, R. A. Arndt, W. J. Briscoe, M. W. Paris and I. I. Strakovsky, Phys. Rev. C 86, 035202 (2012) arXiv:1204.2277 [hep-ph]].

[62] R. J. Furnstahl, N. Klco, D. R. Phillips and S. Wesolowski, Phys. Rev. C 92, no. 2, 024005 (2015) arXiv:1506.01343 [nucl-th]].

[63] H. Denz et al., Phys. Lett. B 633, 209 (2006) nucl-ex/0512006.

[64] I. I. Strakovsky, private communication.

[65] V. Baru, C. Hanhart, M. Hoferichter, B. Kubis, A. Nogga and D. R. Phillips, Nucl. Phys. A 872, 69 (2011) arXiv:1107.5509 [nucl-th]].

[66] E. E. Jenkins and A. V. Manohar, Phys. Lett. B 259, 353 (1991).

[67] T. R. Hemmert, B. R. Holstein and J. Kambor, J. Phys. G 24, 1831 (1998) hep-ph/9712496.

[68] V. Pascalutsa and D. R. Phillips, Phys. Rev. C 67, 055202 (2003) nucl-th/0212024.

[69] V. Bernard, E. Epelbaum, H. Krebs and U.-G. Meißner, Phys. Rev. D 87, no. 5, 054032 (2013) arXiv:1209.2523 [hep-ph]]. 


\section{Appendix C: Tables}

\begin{tabular}{c|rrr}
\hline \hline$Q^{2}$ & HB-NN & HB- $\pi \mathrm{N}$ & \multicolumn{1}{c}{ Cov } \\
\hline \hline$c_{1}$ & $-1.69(4)$ & $-1.60(5)$ & $-2.19(5)$ \\
$c_{2}$ & $3.18(8)$ & $3.63(9)$ & $2.52(7)$ \\
$c_{3}$ & $-6.08(5)$ & $-6.24(5)$ & $-6.25(6)$ \\
$c_{4}$ & $4.61(2)$ & $5.22(3)$ & $4.32(2)$ \\
\hline \hline$\chi_{\pi N}^{2} /$ dof & 0.72 & 0.69 & 0.67 \\
\hline \hline $\bar{\chi}_{\pi N}^{2} /$ dof & 116 & 98 & 413 \\
\hline \hline
\end{tabular}

\begin{tabular}{c|rrr}
\hline \hline$Q^{3}$ & HB-NN & HB- $\pi \mathrm{N}$ & \multicolumn{1}{c}{ Cov } \\
\hline \hline$c_{1}$ & $-1.24(2)$ & $-1.64(2)$ & $-1.55(2)$ \\
$c_{2}$ & $4.89(5)$ & $3.51(3)$ & $3.60(4)$ \\
$c_{3}$ & $-7.25(2)$ & $-6.63(2)$ & $-6.54(2)$ \\
$c_{4}$ & $4.74(4)$ & $4.01(4)$ & $3.86(3)$ \\
$d_{1+2}$ & $3.39(4)$ & $4.37(4)$ & $4.09(4)$ \\
$d_{3}$ & $-3.47(7)$ & $-3.34(7)$ & $-2.50(4)$ \\
$d_{5}$ & $0.00(4)$ & $-0.56(4)$ & $-0.86(4)$ \\
$d_{14-15}$ & $-7.39(13)$ & $-7.49(13)$ & $-6.05(10)$ \\
\hline \hline$\chi_{\pi N}^{2} /$ dof & 1.04 & 1.03 & 0.97 \\
\hline $\bar{\chi}_{\pi N}^{2} /$ dof & 14.6 & 13.0 & 13.5 \\
\hline \hline & & & \\
\hline \hline$Q^{4}$ & HB-NN & HB- $\pi \mathrm{N}$ & Cov \\
\hline \hline$c_{1}$ & $-1.31(8)$ & $-1.15(8)$ & $-0.82(7)$ \\
$c_{2}$ & $1.88(23)$ & $2.39(22)$ & $3.56(16)$ \\
$c_{3}$ & $-4.43(9)$ & $-4.44(9)$ & $-4.59(9)$ \\
$c_{4}$ & $3.24(17)$ & $3.45(17)$ & $3.44(13)$ \\
$d_{1+2}$ & $5.95(9)$ & $5.60(9)$ & $5.43(5)$ \\
$d_{3}$ & $-5.64(6)$ & $-3.84(4)$ & $-4.58(8)$ \\
$d_{5}$ & $-0.11(4)$ & $-0.89(4)$ & $-0.40(4)$ \\
$d_{14-15}$ & $-11.61(9)$ & $-9.45(8)$ & $-9.94(7)$ \\
$e_{14}$ & $0.86(29)$ & $1.28(32)$ & $-0.63(24)$ \\
$e_{15}$ & $-11.36(81)$ & $-13.26(79)$ & $-7.33(45)$ \\
$e_{16}$ & $10.73(95)$ & $8.29(95)$ & $1.86(37)$ \\
$e_{17}$ & $-0.66(46)$ & $-0.73(47)$ & $-0.90(32)$ \\
$e_{18}$ & $4.47(87)$ & $4.17(90)$ & $3.17(45)$ \\
\hline \hline$\chi_{\pi N}^{2} /$ dof & 1.90 & 1.83 & 1.94 \\
\hline \hline $\bar{\chi}_{\pi N}^{2} /$ dof & 4.5 & 4.1 & 4.9 \\
\hline \hline & & & \\
\hline & & & \\
\hline & & & \\
\hline
\end{tabular}

TABLE I: LECs determined from fits at order $Q^{2}, Q^{3}, Q^{4}$ with $T_{\pi}<100 \mathrm{MeV}$. 


\begin{tabular}{|c|c|c|c|c|c|c|c|c|}
\hline HBNN & $c_{1}$ & $c_{2}$ & $c_{3}$ & $c_{4}$ & $d_{1+2}$ & $d_{3}$ & $d_{5}$ & $d_{14-15}$ \\
\hline$c_{1}$ & 6 & 91 & -39 & 23 & -15 & 1 & 6 & 4 \\
\hline$c_{2}$ & 10 & 21 & -73 & 28 & 7 & -6 & -3 & 0 \\
\hline$c_{3}$ & -2 & -7 & 4 & -17 & -43 & 17 & 15 & 7 \\
\hline$c_{4}$ & 2 & 5 & -1 & 16 & -22 & 15 & -4 & 50 \\
\hline$d_{1+2}$ & -2 & 1 & -4 & -4 & 18 & -57 & -4 & -17 \\
\hline$d_{3}$ & 0 & -2 & 2 & 4 & -16 & 44 & -78 & 2 \\
\hline$d_{5}$ & 1 & -1 & 1 & -1 & -1 & -21 & 17 & 17 \\
\hline$d_{14-15}$ & 1 & 0 & 2 & 25 & -9 & 1 & 9 & 163 \\
\hline $\mathrm{HB} \pi \mathrm{N}$ & $c_{1}$ & $c_{2}$ & $c_{3}$ & $c_{4}$ & $d_{1+2}$ & $d_{3}$ & $d_{5}$ & $d_{14-15}$ \\
\hline$c_{1}$ & 4 & 86 & $\begin{array}{l}-1 \\
\end{array}$ & 22 & -15 & 26 & 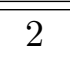 & 14 \\
\hline$c_{2}$ & 6 & 11 & -52 & 29 & 16 & -9 & -3 & 4 \\
\hline$c_{3}$ & 0 & -3 & 3 & -9 & -58 & 31 & 6 & 17 \\
\hline$c_{4}$ & 2 & 3 & -1 & 12 & 2 & 14 & -15 & 52 \\
\hline$d_{1+2}$ & -1 & 2 & -4 & 0 & 15 & -50 & -6 & 1 \\
\hline$d_{3}$ & 1 & -2 & 4 & 3 & -13 & 46 & -82 & 1 \\
\hline$d_{5}$ & 0 & 0 & 0 & -2 & -1 & -24 & 19 & 7 \\
\hline$d_{14-15}$ & 4 & 2 & 4 & 24 & 0 & 1 & 4 & 168 \\
\hline Cov & $c_{1}$ & $c_{2}$ & $c_{3}$ & $c_{4}$ & $d_{1+2}$ & $d_{3}$ & $d_{5}$ & $d_{14-15}$ \\
\hline$c_{1}$ & 6 & 83 & 20 & 27 & 7 & 14 & $\begin{array}{l}-16 \\
\end{array}$ & 21 \\
\hline$c_{2}$ & 7 & 12 & -38 & 31 & 10 & 1 & -12 & 0 \\
\hline$c_{3}$ & 1 & -3 & 5 & -2 & -2 & 22 & -7 & 38 \\
\hline$c_{4}$ & 2 & 4 & 0 & 12 & 10 & 9 & -14 & 46 \\
\hline$d_{1+2}$ & 1 & 1 & 0 & 1 & 17 & -7 & -56 & 19 \\
\hline$d_{3}$ & 1 & 0 & 2 & 1 & -1 & 19 & -77 & -9 \\
\hline$d_{5}$ & -2 & -2 & -1 & -2 & -10 & -14 & 18 & 3 \\
\hline$d_{14-15}$ & 5 & 0 & 9 & 16 & 8 & -4 & 1 & 102 \\
\hline
\end{tabular}

TABLE II: The upper and lower triangle correspond to the correlation and the covariance matrices for the fits at $Q^{3}$. The correlation and covariance values are given in units of $10^{-2}$ and $10^{-4}$, respectively. 


\begin{tabular}{|c|c|c|c|c|c|c|c|c|c|c|c|c|c|}
\hline HB-NN & $c_{1}$ & $c_{2}$ & $c_{3}$ & $c_{4}$ & $d_{1+2}$ & $d_{3}$ & $d_{5}$ & $d_{14-15}$ & $e_{14}$ & $e_{15}$ & $e_{16}$ & $e_{17}$ & $e_{18}$ \\
\hline$c_{1}$ & 61 & 90 & 12 & 39 & 35 & -20 & -28 & -26 & -30 & 38 & -78 & 9 & -35 \\
\hline$c_{2}$ & 162 & 531 & -31 & 38 & 41 & -23 & -35 & -43 & -24 & 58 & -94 & 10 & -35 \\
\hline$c_{3}$ & 8 & -64 & 82 & 3 & -14 & 7 & 16 & 39 & -1 & -56 & 46 & -5 & 1 \\
\hline$c_{4}$ & 52 & 147 & 5 & 288 & 94 & -61 & -65 & -55 & -29 & 29 & -38 & 15 & -86 \\
\hline$d_{1+2}$ & 25 & 87 & -12 & 148 & 85 & -68 & -66 & -56 & -26 & 36 & -43 & 11 & -80 \\
\hline$d_{3}$ & -9 & -31 & 4 & -62 & -38 & 36 & -9 & 42 & 23 & -24 & 25 & -30 & 63 \\
\hline$d_{5}$ & -9 & -34 & 6 & -46 & -26 & -2 & 18 & 37 & 15 & -27 & 36 & 13 & 45 \\
\hline$d_{14-15}$ & -19 & -93 & 34 & -89 & -49 & 24 & 15 & 90 & 25 & -48 & 50 & -42 & 66 \\
\hline$e_{14}$ & -67 & -163 & -3 & -144 & -70 & 39 & 18 & 69 & 835 & -78 & 48 & -20 & 31 \\
\hline$e_{15}$ & 240 & 1077 & -412 & 395 & 268 & -116 & -93 & -367 & -1832 & 6534 & -81 & 21 & -32 \\
\hline$e_{16}$ & -579 & -2064 & 400 & -613 & -381 & 142 & 145 & 454 & 1309 & -6269 & 9065 & -16 & 38 \\
\hline$e_{17}$ & 33 & 107 & -19 & 121 & 47 & -82 & 25 & -183 & -268 & 774 & -689 & 2140 & -62 \\
\hline$e_{18}$ & -236 & -696 & 5 & -1271 & -639 & 326 & 164 & 539 & 767 & -2220 & 3096 & -2502 & 7518 \\
\hline $\mathrm{HB}-\pi \mathrm{N}$ & $c_{1}$ & $c_{2}$ & $c_{3}$ & $c_{4}$ & $d_{1+2}$ & $d_{3}$ & $d_{5}$ & $d_{14-15}$ & $e_{14}$ & $e_{15}$ & $e_{16}$ & $e_{17}$ & $e_{18}$ \\
\hline$c_{1}$ & 60 & 93 & 8 & 39 & 37 & -20 & -33 & -19 & -15 & 32 & -80 & 12 & -36 \\
\hline$c_{2}$ & 159 & 494 & -29 & 39 & 43 & -22 & -40 & -34 & -8 & 47 & -93 & 13 & -37 \\
\hline$c_{3}$ & 5 & -58 & 80 & 2 & -16 & 6 & 19 & 39 & -8 & -51 & 46 & -4 & 1 \\
\hline$c_{4}$ & 53 & 149 & 3 & 300 & 94 & -62 & -72 & -51 & -18 & 23 & -38 & 19 & -87 \\
\hline$d_{1+2}$ & 25 & 85 & -13 & 144 & 78 & -62 & -80 & -49 & -9 & 26 & -43 & 11 & -79 \\
\hline$d_{3}$ & -6 & -20 & 2 & -45 & -23 & 17 & 4 & 42 & 20 & -23 & 25 & -33 & 65 \\
\hline$d_{5}$ & -11 & -38 & 7 & -53 & -30 & 1 & 18 & 35 & -1 & -20 & 38 & 8 & 53 \\
\hline$d_{14-15}$ & -11 & -58 & 26 & -68 & -33 & 13 & 11 & 58 & 25 & -47 & 46 & -45 & 63 \\
\hline$e_{14}$ & -37 & -59 & -23 & -101 & -25 & 26 & -2 & 61 & 1007 & -78 & 35 & -20 & 21 \\
\hline$e_{15}$ & 196 & 825 & -359 & 318 & 182 & -74 & -66 & -281 & -1957 & 6273 & -75 & 22 & -27 \\
\hline$e_{16}$ & -583 & -1959 & 388 & -618 & -358 & 96 & 155 & 328 & 1040 & -5623 & 8954 & -19 & 38 \\
\hline$e_{17}$ & 42 & 133 & -18 & 153 & 47 & -65 & 16 & -161 & -301 & 833 & -830 & 2191 & -64 \\
\hline$e_{18}$ & -250 & -732 & 11 & -1356 & -629 & 240 & 202 & 433 & 611 & -1949 & 3261 & -2681 & 8066 \\
\hline Cov & $c_{1}$ & $c_{2}$ & $c_{3}$ & $c_{4}$ & $d_{1+2}$ & $d_{3}$ & $d_{5}$ & $d_{14-15}$ & $e_{14}$ & $e_{15}$ & $e_{16}$ & $e_{17}$ & $e_{18}$ \\
\hline$c_{1}$ & 49 & 92 & 48 & 35 & -11 & 80 & -78 & 3 & -37 & 1 & $\begin{array}{l}-63 \\
\end{array}$ & 11 & -36 \\
\hline$c_{2}$ & 104 & 266 & 9 & 38 & 5 & 62 & -70 & -19 & -18 & 15 & -82 & 11 & -40 \\
\hline$c_{3}$ & 30 & 14 & 82 & 10 & -34 & 62 & -40 & 45 & -45 & -37 & 24 & 0 & -9 \\
\hline$c_{4}$ & 32 & 81 & 12 & 173 & 70 & -6 & -41 & -43 & -25 & 18 & -39 & -14 & -80 \\
\hline$d_{1+2}$ & -3 & 4 & -15 & 43 & 22 & -51 & -14 & -47 & -1 & 31 & -24 & -1 & -61 \\
\hline$d_{3}$ & 43 & 78 & 43 & -6 & -18 & 59 & -77 & 31 & -29 & -19 & -30 & 1 & 5 \\
\hline$d_{5}$ & -23 & -49 & -15 & -23 & -3 & -25 & 18 & 2 & 32 & -3 & 50 & 1 & 35 \\
\hline$d_{14-15}$ & 2 & -21 & 28 & -39 & -15 & 16 & 1 & 48 & -3 & -33 & 38 & -21 & 52 \\
\hline$e_{14}$ & -61 & -71 & -98 & -79 & -2 & -53 & 32 & -5 & 565 & -65 & 41 & -14 & 27 \\
\hline$e_{15}$ & 4 & 108 & -151 & 105 & 64 & -64 & -6 & -101 & -695 & 2006 & -66 & 17 & -22 \\
\hline$e_{16}$ & -162 & -491 & 80 & -187 & -42 & -84 & 78 & 97 & 359 & -1094 & 1353 & -17 & 43 \\
\hline$e_{17}$ & 24 & 58 & 0 & -57 & -1 & 3 & 1 & -45 & -103 & 235 & -196 & 1010 & -46 \\
\hline$e_{18}$ & -114 & -290 & -35 & -472 & -128 & 15 & 65 & 159 & 284 & -442 & 703 & -659 & 1996 \\
\hline
\end{tabular}

TABLE III: The upper and lower triangle correspond to the correlation and the covariance matrices for the fits at $Q^{4}$. The correlation and covariance values are given in units of $10^{-2}$ and $10^{-4}$, respectively. 


\begin{tabular}{|c|c|c|c|}
\hline$Q^{2}+\delta^{1}$ & HB-NN & $\mathrm{HB}-\pi \mathrm{N}$ & $\mathrm{Cov}$ \\
\hline$c_{1}$ & $-1.02(3)$ & $-0.84(4)$ & $-0.88(3)$ \\
\hline$c_{2}$ & $0.26(6)$ & $0.85(6)$ & $0.64(4)$ \\
\hline$c_{3}$ & $-0.98(3)$ & $-1.13(3)$ & $-1.00(3)$ \\
\hline$c_{4}$ & $0.48(4)$ & $1.09(3)$ & $1.00(3)$ \\
\hline$\overline{\chi_{\pi N}^{2} / \operatorname{dof}}$ & 0.51 & 0.50 & 0.53 \\
\hline$\overline{\bar{\chi}_{\pi N}^{2} / \text { dof }}$ & 11 & 3.5 & 3.3 \\
\hline$\overline{Q^{3}+\delta^{1}}$ & HB-NN & $\mathrm{HB}-\pi \mathrm{N}$ & $\mathrm{Cov}$ \\
\hline$c_{1}$ & $-1.35(2)$ & $-1.45(1)$ & $-1.13(1)$ \\
\hline$c_{2}$ & $1.27(3)$ & $0.89(2)$ & $1.24(2)$ \\
\hline$c_{3}$ & $-2.71(1)$ & $-2.52(1)$ & $-2.29(1)$ \\
\hline$c_{4}$ & $2.06(2)$ & $1.77(2)$ & $1.73(2)$ \\
\hline$d_{1+2}$ & $-0.47(3)$ & $-0.08(3)$ & $0.24(2)$ \\
\hline$d_{3}$ & $-0.72(6)$ & $-0.59(5)$ & $-0.68(3)$ \\
\hline$d_{5}$ & $0.71(4)$ & $0.43(3)$ & $0.29(3)$ \\
\hline$d_{14-15}$ & $-0.16(6)$ & $-0.40(6)$ & $-0.37(4)$ \\
\hline$\overline{\chi_{\pi N}^{2} / \text { dof }}$ & 0.98 & 1.09 & 1.08 \\
\hline$\overline{\overline{\bar{\chi}_{\pi N}}} / \mathrm{dof}$ & 2.2 & 2.2 & 2.2 \\
\hline$\overline{Q^{4}+\delta^{1}}$ & HB-NN & $\mathrm{HB}-\pi \mathrm{N}$ & Cov \\
\hline$c_{1}$ & $-1.34(6)$ & $-1.19(6)$ & $-1.15(5)$ \\
\hline$c_{2}$ & $0.94(17)$ & $1.34(15)$ & $1.57(10)$ \\
\hline$c_{3}$ & $-2.35(5)$ & $-2.33(5)$ & $-2.54(5)$ \\
\hline$c_{4}$ & $2.39(13)$ & $2.45(12)$ & $2.61(10)$ \\
\hline$d_{1+2}$ & $1.24(7)$ & $1.41(6)$ & $1.29(3)$ \\
\hline$d_{3}$ & $-1.79(5)$ & $-1.16(3)$ & $-1.83(5)$ \\
\hline$d_{5}$ & $0.38(3)$ & $-0.07(3)$ & $0.37(3)$ \\
\hline$d_{14-15}$ & $-1.92(7)$ & $-1.67(5)$ & $-2.22(5)$ \\
\hline$e_{14}$ & $1.20(20)$ & $1.00(18)$ & $0.49(13)$ \\
\hline$e_{15}$ & $-2.74(54)$ & $-2.72(51)$ & $-1.07(29)$ \\
\hline$e_{16}$ & $1.30(62)$ & $-0.91(63)$ & $-1.54(22)$ \\
\hline$e_{17}$ & $-0.83(30)$ & $-0.49(29)$ & $-0.94(19)$ \\
\hline$e_{18}$ & $-1.64(61)$ & $-1.50(58)$ & $-1.22(29)$ \\
\hline$\overline{\chi_{\pi N}^{2} / \operatorname{dof}}$ & 1.64 & 1.72 & 1.71 \\
\hline$\overline{\overline{\chi_{\pi N}}} 2 /$ dof & 2.0 & 2.0 & 2.0 \\
\hline
\end{tabular}

TABLE IV: LECs determined from fits at order $Q^{2}+\delta^{1}, Q^{3}+\delta^{1}, Q^{4}+\delta^{1}$ with $T_{\pi}<100 \mathrm{MeV}$. 


\begin{tabular}{|c|c|c|c|c|c|c|c|c|}
\hline HB-NN & $c_{1}$ & $c_{2}$ & $c_{3}$ & $c_{4}$ & $d_{1+2}$ & $d_{3}$ & $d_{5}$ & $d_{14-15}$ \\
\hline$c_{1}$ & 3 & 88 & -26 & 34 & 17 & -36 & 34 & -1 \\
\hline$c_{2}$ & 5 & 11 & -68 & 42 & 32 & -46 & 34 & -8 \\
\hline$c_{3}$ & -1 & -3 & 2 & -23 & -38 & 36 & -19 & 11 \\
\hline$c_{4}$ & 1 & 3 & -1 & 5 & 13 & -25 & 17 & -39 \\
\hline$d_{1+2}$ & 1 & 3 & -2 & 1 & 9 & -64 & 16 & -8 \\
\hline$d_{3}$ & -4 & -9 & 3 & -3 & -12 & 39 & -85 & 25 \\
\hline$d_{5}$ & 2 & 4 & -1 & 1 & 2 & -19 & 13 & -19 \\
\hline$d_{14-15}$ & 0 & -2 & 1 & -5 & -2 & 10 & -4 & 40 \\
\hline$\overline{\mathrm{HB}}-\pi \mathrm{N}$ & $c_{1}$ & $c_{2}$ & $c_{3}$ & $c_{4}$ & $d_{1+2}$ & $d_{3}$ & $d_{5}$ & $d_{14-15}$ \\
\hline$c_{1}$ & 2 & 81 & 12 & 23 & 5 & -19 & 19 & 18 \\
\hline$c_{2}$ & 2 & 4 & -46 & 30 & 24 & -31 & 21 & 11 \\
\hline$c_{3}$ & 0 & -1 & 1 & -5 & -32 & 26 & -9 & 9 \\
\hline$c_{4}$ & 0 & 1 & 0 & 2 & 13 & -2 & -5 & -18 \\
\hline$d_{1+2}$ & 0 & 1 & -1 & 1 & 7 & -49 & -1 & 11 \\
\hline$d_{3}$ & -1 & -3 & 2 & 0 & -7 & 26 & -85 & 5 \\
\hline$d_{5}$ & 1 & 1 & 0 & 0 & 0 & -15 & 11 & -7 \\
\hline$d_{14-15}$ & 1 & 1 & 1 & -2 & 2 & 2 & -1 & 31 \\
\hline Cov & $c_{1}$ & $c_{2}$ & $c_{3}$ & $c_{4}$ & $d_{1+2}$ & $d_{3}$ & $d_{5}$ & $d_{14-15}$ \\
\hline$c_{1}$ & 2 & 80 & 17 & 18 & -1 & -15 & 16 & 30 \\
\hline$c_{2}$ & 2 & 4 & -43 & 26 & 13 & -24 & 14 & 19 \\
\hline$c_{3}$ & 0 & -1 & 2 & -6 & -22 & 21 & -4 & 18 \\
\hline$c_{4}$ & 0 & 1 & 0 & 2 & 11 & 4 & -10 & -14 \\
\hline$d_{1+2}$ & 0 & 1 & -1 & 0 & 5 & -21 & -38 & 16 \\
\hline$d_{3}$ & -1 & -2 & 1 & 0 & -1 & 10 & -81 & 1 \\
\hline$d_{5}$ & 1 & 1 & 0 & 0 & -2 & -7 & 8 & -5 \\
\hline$d_{14-15}$ & 2 & 2 & 1 & -1 & 2 & 0 & -1 & 19 \\
\hline
\end{tabular}

TABLE V: The upper and lower triangle correspond to the correlation and the covariance matrices for the fits at $Q^{3}+\delta^{1}$. The correlation and covariance values are given in units of $10^{-2}$ and $10^{-4}$, respectively. 


\begin{tabular}{|c|c|c|c|c|c|c|c|c|c|c|c|c|c|}
\hline HB-NN & $c_{1}$ & $c_{2}$ & $c_{3}$ & $c_{4}$ & $d_{1+2}$ & $d_{3}$ & $d_{5}$ & $d_{14-15}$ & $e_{14}$ & $e_{15}$ & $e_{16}$ & $e_{17}$ & $e_{18}$ \\
\hline$c_{1}$ & 38 & 94 & 23 & 44 & 39 & -17 & -31 & -20 & -7 & 20 & -78 & 11 & -40 \\
\hline$c_{2}$ & 97 & 279 & -11 & 42 & 42 & -20 & -33 & -33 & -9 & 38 & -92 & 13 & -41 \\
\hline$c_{3}$ & 8 & -10 & 30 & 10 & -4 & 10 & -4 & 32 & 18 & -59 & 35 & -5 & -6 \\
\hline$c_{4}$ & 35 & 91 & 7 & 166 & 95 & -53 & -61 & -57 & -25 & 26 & -43 & 14 & -88 \\
\hline$d_{1+2}$ & 17 & 48 & -1 & 84 & 47 & -66 & -55 & -58 & -28 & 33 & -45 & 12 & -83 \\
\hline$d_{3}$ & -5 & -17 & 3 & -36 & -24 & 27 & -26 & 54 & 21 & -23 & 23 & -37 & 60 \\
\hline$d_{5}$ & -6 & -18 & -1 & -26 & -13 & -4 & 11 & 18 & 15 & -19 & 34 & 22 & 39 \\
\hline$d_{14-15}$ & -9 & -40 & 13 & -52 & -28 & 20 & 4 & 51 & 24 & -41 & 43 & -50 & 69 \\
\hline$e_{14}$ & -8 & -30 & 20 & -63 & -38 & 21 & 10 & 34 & 382 & -86 & 41 & -9 & 22 \\
\hline$e_{15}$ & 66 & 341 & -175 & 178 & 122 & -65 & -34 & -156 & -902 & 2911 & -71 & 14 & -25 \\
\hline$e_{16}$ & -300 & -948 & 118 & -339 & -191 & 73 & 70 & 189 & 498 & -2350 & 3815 & -17 & 42 \\
\hline$e_{17}$ & 21 & 67 & -8 & 54 & 26 & -58 & 23 & -108 & -52 & 224 & -310 & 920 & -59 \\
\hline$e_{18}$ & -154 & -417 & -19 & -695 & -350 & 192 & 80 & 303 & 260 & -836 & 1588 & -1095 & 3772 \\
\hline $\mathrm{HB}-\pi \mathrm{N}$ & $c_{1}$ & $c_{2}$ & $c_{3}$ & $c_{4}$ & $d_{1+2}$ & $d_{3}$ & $d_{5}$ & $d_{14-15}$ & $e_{14}$ & $e_{15}$ & $e_{16}$ & $e_{17}$ & $e_{18}$ \\
\hline$c_{1}$ & 37 & 95 & 14 & 43 & 41 & -19 & -35 & -16 & -8 & 25 & -81 & 10 & -40 \\
\hline$c_{2}$ & 89 & 236 & -17 & 41 & 43 & -22 & -35 & -29 & -10 & 42 & -93 & 12 & -39 \\
\hline$c_{3}$ & 5 & -14 & 28 & 12 & -1 & 8 & -3 & 34 & 16 & -60 & 39 & -6 & -7 \\
\hline$c_{4}$ & 33 & 79 & 8 & 156 & 95 & -53 & -71 & -54 & -24 & 23 & -40 & 12 & -88 \\
\hline$d_{1+2}$ & 15 & 40 & 0 & 72 & 37 & -58 & -74 & -51 & -27 & 31 & -44 & 8 & -83 \\
\hline$d_{3}$ & -4 & -11 & 1 & -22 & -11 & 11 & -11 & 48 & 17 & -20 & 23 & -35 & 61 \\
\hline$d_{5}$ & -7 & -17 & -1 & -27 & -14 & -1 & 10 & 26 & 21 & -24 & 37 & 17 & 50 \\
\hline$d_{14-15}$ & -5 & -23 & 9 & -35 & -16 & 8 & 4 & 28 & 21 & -37 & 38 & -49 & 67 \\
\hline$e_{14}$ & -9 & -28 & 16 & -55 & -30 & 10 & 12 & 21 & 331 & -84 & 39 & -3 & 19 \\
\hline$e_{15}$ & 78 & 324 & -160 & 147 & 95 & -33 & -37 & -100 & -779 & 2587 & -71 & 9 & -22 \\
\hline$e_{16}$ & -312 & -899 & 130 & -317 & -170 & 48 & 71 & 127 & 450 & -2290 & 3970 & -14 & 39 \\
\hline$e_{17}$ & 17 & 54 & -9 & 43 & 14 & -32 & 15 & -73 & -16 & 135 & -253 & 814 & -57 \\
\hline$e_{18}$ & -142 & -354 & -20 & -642 & -293 & 115 & 91 & 206 & 202 & -643 & 1439 & -949 & 3421 \\
\hline Cov & $c_{1}$ & $c_{2}$ & $c_{3}$ & $c_{4}$ & $d_{1+2}$ & $d_{3}$ & $d_{5}$ & $d_{14-15}$ & $e_{14}$ & $e_{15}$ & $e_{16}$ & $e_{17}$ & $e_{18}$ \\
\hline$c_{1}$ & 27 & 94 & 55 & 41 & 1 & 72 & -71 & 3 & -14 & -19 & -65 & 5 & -41 \\
\hline$c_{2}$ & 47 & 95 & 24 & 41 & 8 & 59 & -64 & -14 & -5 & -8 & -79 & 9 & -44 \\
\hline$c_{3}$ & 14 & 11 & 24 & 25 & -8 & 58 & -51 & 34 & -22 & -43 & 10 & -9 & -19 \\
\hline$c_{4}$ & 20 & 39 & 12 & 93 & 74 & -5 & -41 & -50 & -28 & 9 & -42 & -22 & -84 \\
\hline$d_{1+2}$ & 0 & 2 & -1 & 21 & 9 & -43 & -19 & -49 & -22 & 24 & -23 & -5 & -68 \\
\hline$d_{3}$ & 18 & 27 & 13 & -2 & -6 & 23 & -79 & 38 & -2 & -31 & -29 & -7 & 9 \\
\hline$d_{5}$ & -11 & -19 & -7 & -12 & -2 & -11 & 9 & -6 & 18 & 13 & 45 & 12 & 31 \\
\hline$d_{14-15}$ & 1 & -7 & 8 & -24 & -7 & 9 & -1 & 25 & 7 & -27 & 33 & -23 & 59 \\
\hline$e_{14}$ & -10 & -6 & -14 & -36 & -9 & -1 & 7 & 5 & 181 & -78 & 46 & 11 & 17 \\
\hline$e_{15}$ & -28 & -23 & -60 & 26 & 20 & -43 & 11 & -38 & -301 & 820 & -52 & -2 & -4 \\
\hline$e_{16}$ & -72 & -167 & 11 & -86 & -14 & -30 & 29 & 35 & 133 & -323 & 465 & -7 & 42 \\
\hline$e_{17}$ & 5 & 16 & -8 & -39 & -3 & -6 & 7 & -21 & 27 & -11 & -30 & 344 & -33 \\
\hline$e_{18}$ & -61 & -123 & -27 & -234 & -59 & 13 & 27 & 86 & 67 & -36 & 263 & -178 & 840 \\
\hline
\end{tabular}

TABLE VI: The upper and lower triangle correspond to the correlation and the covariance matrices for the fits at $Q^{4}+\delta^{1}$. The correlation and covariance values are given in units of $10^{-2}$ and $10^{-4}$, respectively. 


\begin{tabular}{c|c|c|c|c}
\hline \hline$Q^{3}$ & HB-NN & HB- $\pi \mathrm{N}$ & Cov & RS \\
\hline \hline$d_{00}^{+}\left[M_{\pi}^{-1}\right]$ & $-2.34(4)(1.97)$ & $-1.44(3)(95)$ & $-1.72(3)(50)$ & $-1.36(3)$ \\
$d_{10}^{+}\left[M_{\pi}^{-3}\right]$ & $2.20(3)(3.06)$ & $1.32(2)(1.98)$ & $1.83(2)(1.08)$ & $1.16(2)$ \\
$d_{01}^{+}\left[M_{\pi}^{-3}\right]$ & $1.75(1)(96)$ & $1.55(1)(85)$ & $1.68(1)(71)$ & $1.16(2)$ \\
$d_{20}^{+}\left[M_{\pi}^{-5}\right]$ & $0.22(0)(1.07)$ & $0.22(0)(1.07)$ & $0.06(0)(48)$ & $0.196(3)$ \\
$d_{11}^{+}\left[M_{\pi}^{-5}\right]$ & $0.07(0)(57)$ & $0.07(0)(73)$ & $0.02(0)(41)$ & $0.185(3)$ \\
$d_{02}^{+}\left[M_{\pi}^{-5}\right]$ & $0.035(0)(8)$ & $0.035(0)(18)$ & $0.017(0)(21)$ & $0.0336(6)$ \\
$b_{00}^{+}\left[M_{\pi}^{-3}\right]$ & $-10.1(2)(4.9)$ & $-10.2(2)(8.8)$ & $-8.0(1)(1.9)$ & $-3.45(7)$ \\
$d_{00}^{-}\left[M_{\pi}^{-2}\right]$ & $1.78(2)(63)$ & $1.76(2)(1.04)$ & $1.53(1)(14)$ & $1.41(1)$ \\
$d_{10}^{-}\left[M_{\pi}^{-4}\right]$ & $-0.70(1)(99)$ & $-0.67(1)(1.44)$ & $-0.40(1)(20)$ & $-0.159(4)$ \\
$d_{01}^{-}\left[M_{\pi}^{-4}\right]$ & $-0.35(0)(14)$ & $-0.44(0)(38)$ & $-0.35(0)(10)$ & $-0.141(5)$ \\
$b_{00}^{-}\left[M_{\pi}^{-2}\right]$ & $15.3(2)(8.9)$ & $12.2(2)(5.5)$ & $13.8(1)(5.8)$ & $10.49(11)$ \\
$b_{10}^{-}\left[M_{\pi}^{-4}\right]$ & $0.97(0)(4.79)$ & $0.97(0)(5.32)$ & $0.34(0)(3.79)$ & $1.00(3)$ \\
$b_{01}^{-}\left[M_{\pi}^{-4}\right]$ & $0.19(0)(19)$ & $0.19(0)(28)$ & $0.06(0)(32)$ & $0.21(2)$ \\
$a_{0+}^{+}\left[M_{\pi}^{-1} 10^{-3}\right]$ & $80.7(4)(2.1)$ & $81.2(4)(1.0)$ & $81.4(4)(2.0)$ & $85.4(9)$ \\
$a_{0+}^{-}\left[M_{\pi}^{-3} 10^{-3}\right]$ & $4.6(6)(3.8)$ & $6.4(6)(3.5)$ & $7.1(7)(7.1)$ & $-0.9(1.4)$ \\
\hline \hline \multicolumn{5}{|c}{} \\
\hline \hline
\end{tabular}

TABLE VII: Subtreshold and threshold parameters predicted at order $Q^{3}$ and $Q^{4}$ in comparison with RS values. The statistical and theoretical uncertainties are given in the first and second bracket, respectively. 


\begin{tabular}{|c|c|c|c|c|}
\hline$Q^{3}+\delta^{1}$ & HB-NN & $\mathrm{HB}-\pi \mathrm{N}$ & $\mathrm{Cov}$ & $\mathrm{RS}$ \\
\hline$d_{00}^{+}\left[M_{\pi}^{-1}\right]$ & $-1.09(3)(34)$ & $-0.85(2)(14)$ & $-1.33(2)(18)$ & $-1.36(3)$ \\
\hline$d_{10}^{+}\left[M_{\pi}^{-3}\right]$ & $0.72(2)(48)$ & $0.48(1)(21)$ & $1.15(1)(31)$ & $1.16(2)$ \\
\hline$d_{01}^{+}\left[M_{\pi}^{-3}\right]$ & $1.23(0)(23)$ & $1.17(0)(21)$ & $1.25(0)(15)$ & $1.16(2)$ \\
\hline$d_{20}^{+}\left[M_{\pi}^{-5}\right]$ & $0.40(0)(13)$ & $0.40(0)(16)$ & $0.24(0)(14)$ & $0.196(3)$ \\
\hline$d_{11}^{+}\left[M_{\pi}^{-5}\right]$ & $0.24(0)(14)$ & $0.24(0)(21)$ & $0.19(0)(10)$ & $0.185(3)$ \\
\hline$d_{02}^{+}\left[M_{\pi}^{-5}\right]$ & $0.021(0)(19)$ & $0.021(0)(25)$ & $0.005(0)(19)$ & $0.0336(6)$ \\
\hline$b_{00}^{+}\left[M_{\pi}^{-3}\right]$ & $-6.0(1)(4.1)$ & $-6.3(1)(5.5)$ & $-5.8(1)(2.0)$ & $-3.45(7)$ \\
\hline$d_{00}^{-}\left[M_{\pi}^{-2}\right]$ & $1.63(1)(58)$ & $1.60(1)(77)$ & $1.54(1)(20)$ & $1.41(1)$ \\
\hline$d_{10}^{-}\left[M_{\pi}^{-4}\right]$ & $-0.42(1)(70)$ & $-0.39(1)(94)$ & $-0.29(1)(20)$ & $-0.159(4)$ \\
\hline$d_{01}^{-}\left[M_{\pi}^{-4}\right]$ & $-0.22(0)(8)$ & $-0.26(0)(21)$ & $-0.22(0)(5)$ & $-0.141(5)$ \\
\hline$b_{00}^{-}\left[M_{\pi}^{-2}\right]$ & $9.90(9)(60)$ & $8.67(7)(77)$ & $10.81(7)(63)$ & $10.49(11)$ \\
\hline$b_{10}^{-}\left[M_{\pi}^{-4}\right]$ & $1.91(0)(44)$ & $1.91(0)(97)$ & $1.28(0)(68)$ & $1.00(3)$ \\
\hline$b_{01}^{-}\left[M_{\pi}^{-4}\right]$ & $0.07(0)(25)$ & $0.07(0)(21)$ & $-0.07(0)(36)$ & $0.21(2)$ \\
\hline$a_{0+}^{+}\left[M_{\pi}^{-1} 10^{-3}\right]$ & $86.0(3)(1.0)$ & $86.5(3)(2.6)$ & $86.2(3)(1.0)$ & $85.4(9)$ \\
\hline$a_{0+}^{-}\left[M_{\pi}^{-3} 10^{-3}\right]$ & $4.0(5)(3.2)$ & $4.1(5)(3.0)$ & $2.0(5)(3.4)$ & $-0.9(1.4)$ \\
\hline$Q^{4}+\delta^{1}$ & $\mathrm{HB}-\mathrm{NN}$ & $\mathrm{HB}-\pi \mathrm{N}$ & $\mathrm{Cov}$ & $\mathrm{RS}$ \\
\hline $\bar{~} d_{00}^{+}\left[M_{\pi}^{-1}\right]$ & $-0.75(8)(8)$ & $-0.88(9)(3)$ & $-1.15(6)(4)$ & $-1.36(3)$ \\
\hline$d_{10}^{+}\left[M_{\pi}^{-3}\right]$ & $0.23(14)(11)$ & $0.43(14)(5)$ & $0.84(7)(7)$ & $1.16(2)$ \\
\hline$d_{01}^{+}\left[M_{\pi}^{-3}\right]$ & $1.00(3)(5)$ & $0.96(3)(5)$ & $1.10(2)(3)$ & $1.16(2)$ \\
\hline$d_{20}^{+}\left[M_{\pi}^{-5}\right]$ & $0.53(6)(3)$ & $0.56(6)(4)$ & $0.37(2)(3)$ & $0.196(3)$ \\
\hline$d_{11}^{+}\left[M_{\pi}^{-5}\right]$ & $0.37(3)(3)$ & $0.44(3)(5)$ & $0.29(1)(2)$ & $0.185(3)$ \\
\hline$d_{02}^{+}\left[M_{\pi}^{-5}\right]$ & $0.040(5)(4)$ & $0.046(5)(6)$ & $0.025(3)(4)$ & $0.0336(6)$ \\
\hline$b_{00}^{+}\left[M_{\pi}^{-3}\right]$ & $-1.95(13)(94)$ & $-0.74(14)(1.29)$ & $-3.75(6)(47)$ & $-3.45(7)$ \\
\hline$d_{00}^{-}\left[M_{\pi}^{-2}\right]$ & $1.04(2)(14)$ & $0.83(2)(18)$ & $1.33(1)(5)$ & $1.41(1)$ \\
\hline$d_{10}^{-}\left[M_{\pi}^{-4}\right]$ & $0.29(3)(16)$ & $0.54(2)(22)$ & $-0.09(1)(5)$ & $-0.159(4)$ \\
\hline$d_{01}^{-}\left[M_{\pi}^{-4}\right]$ & $-0.148(3)(18)$ & $-0.054(2)(47)$ & $-0.178(2)(12)$ & $-0.141(5)$ \\
\hline$b_{00}^{-}\left[M_{\pi}^{-2}\right]$ & $9.30(54)(14)$ & $9.27(52)(18)$ & $10.82(38)(15)$ & $10.49(11)$ \\
\hline$b_{10}^{-}\left[M_{\pi}^{-4}\right]$ & $2.13(35)(10)$ & $2.88(33)(23)$ & $1.95(17)(16)$ & $1.00(3)$ \\
\hline$b_{01}^{-}\left[M_{\pi}^{-4}\right]$ & $0.32(10)(6)$ & $0.27(10)(5)$ & $0.29(6)(8)$ & $0.21(2)$ \\
\hline$a_{0+}^{+}\left[M_{\pi}^{-1} 10^{-3}\right]$ & $85.9(2)(3)$ & $85.3(2)(6)$ & $86.8(2)(3)$ & $85.4(9)$ \\
\hline$a_{0+}^{-}\left[M_{\pi}^{-3} 10^{-3}\right]$ & $3.0(8)(7)$ & $2.7(8)(7)$ & $2.0(7)(8)$ & $-0.9(1.4)$ \\
\hline
\end{tabular}

TABLE VIII: Subtreshold and threshold parameters predicted at order $Q^{3}+\delta^{1}$ and $Q^{4}+\delta^{1}$ in comparison with RS values. The statistical and theoretical uncertainties are given in the first and second bracket, respectively. 


\section{Appendix D: Figures}

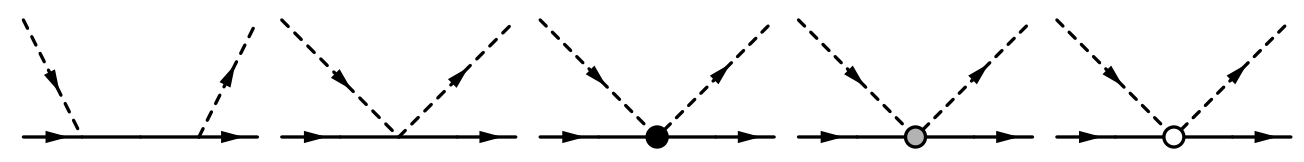

FIG. 1: Tree graphs for the reaction $\pi N \rightarrow \pi N$. The black/gray/white blob denotes an insertion of the $c_{i} / d_{i} / e_{i}$ - vertices. Dashed and solid lines refer to pions and nucleons, respectively. Crossed diagrams are not shown.
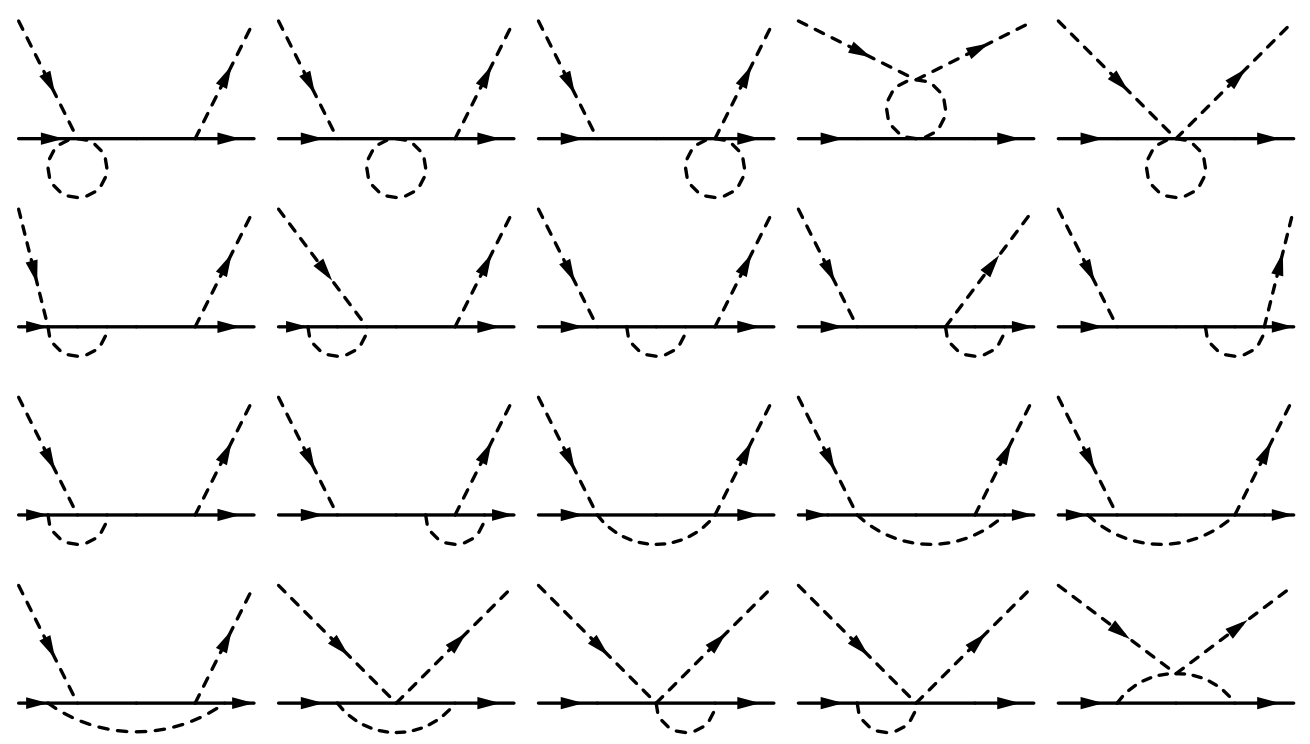

FIG. 2: One-loop graphs for the reaction $\pi N \rightarrow \pi N$. For notation see Fig. 1 .

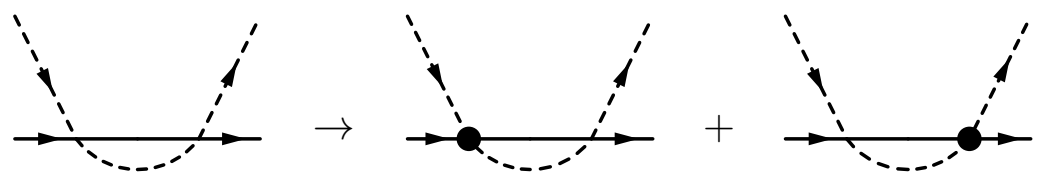

FIG. 3: Transition from leading to next-to-leading order loop graphs. For notation see Fig. 1.

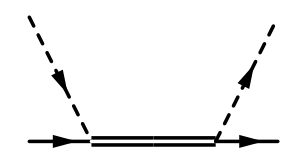

FIG. 4: Leading-order $\Delta$ pole diagram. The double solid line refers to $\Delta$. For notation see Fig. 1 . 


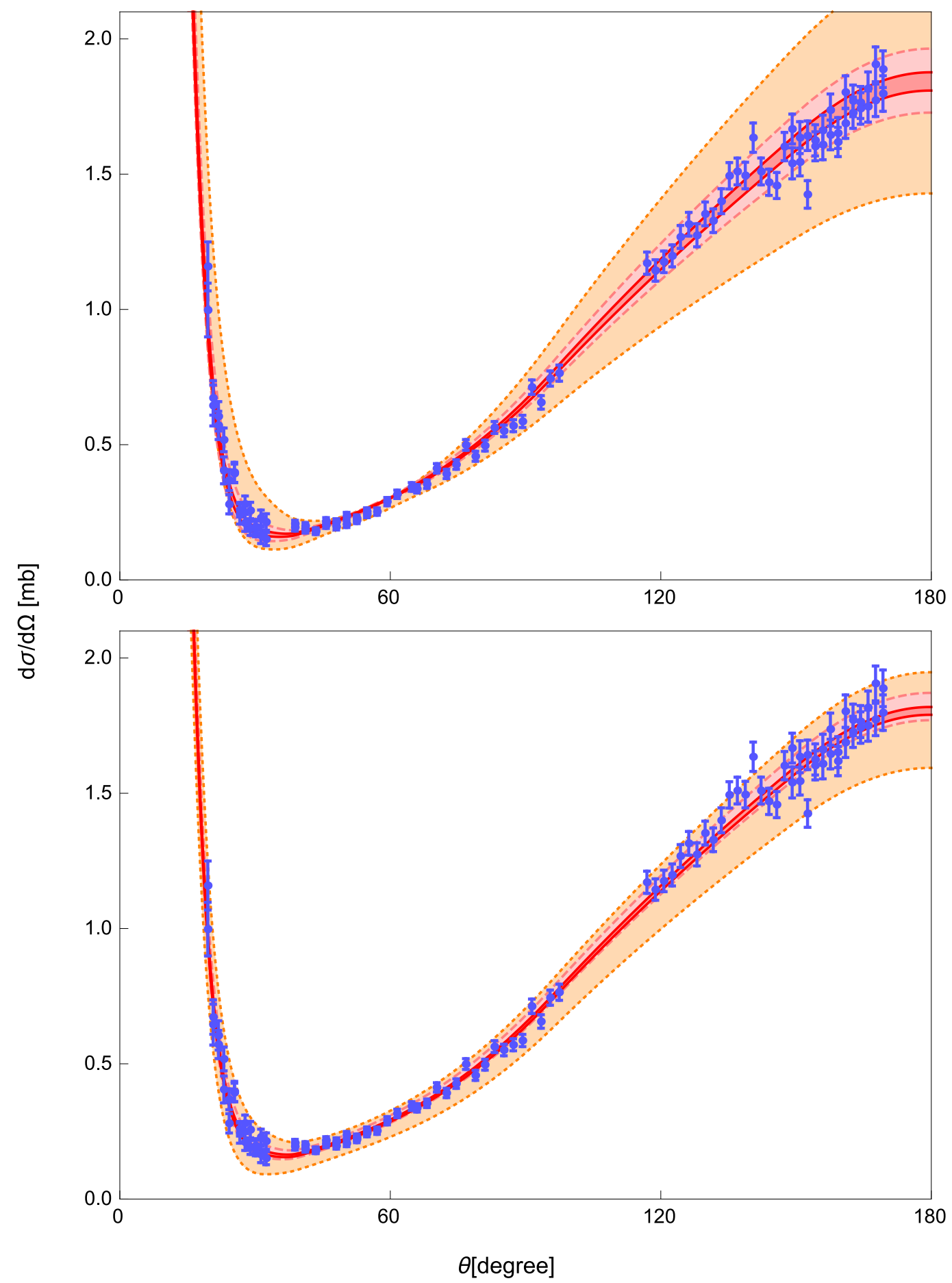

FIG. 5: $\pi^{+} p \rightarrow \pi^{+} p$ differential cross section at $T_{\pi}=43.3 \mathrm{MeV}$ as a representative example of the quality of our fits (carried out to all available data for $T_{\pi}<100 \mathrm{MeV}$ ). In the upper panel, the orange, pink and red (dotted, dashed and solid) bands refer to $Q^{2}, Q^{3}$ and $Q^{4}$ results in the covariant approach including theoretical uncertainties, respectively. In the lower panel the orange, pink and red (dotted, dashed and solid) bands refer to $Q^{2}+\delta^{1}, Q^{3}+\delta^{1}$ and $Q^{4}+\delta^{1}$ results in the covariant approach including theoretical uncertainties, respectively. Experimental data of Ref. 63. are taken from the GWU-SAID data base [61]. 

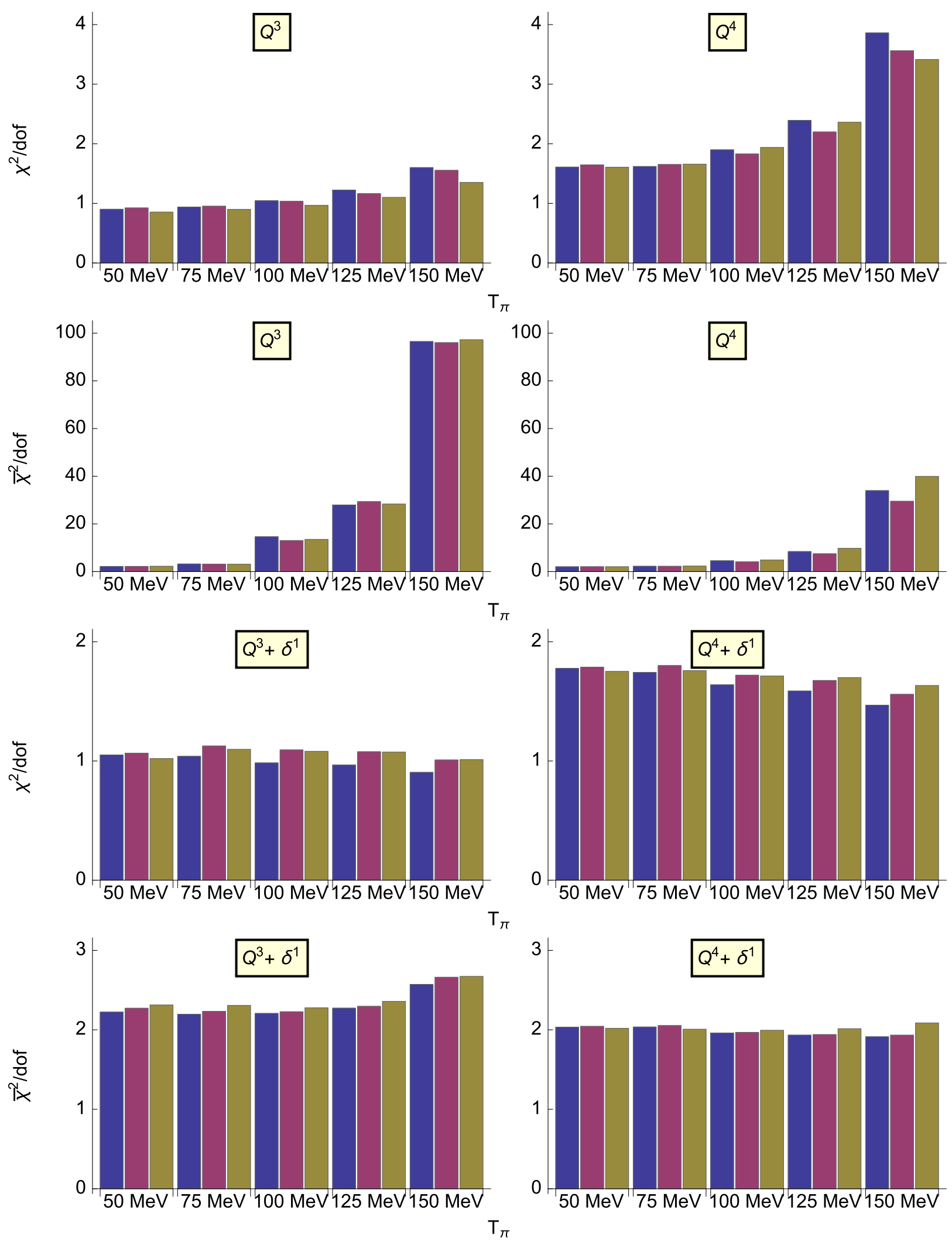

FIG. 6: Reduced $\chi^{2}$ (with theoretical error) and $\bar{\chi}^{2}$ (without theoretical error) for fits up to various maximum energy $T_{\pi}$. The blue/red/green bars denote the results for the HB-NN/HB- $\pi$ N/Cov counting. 

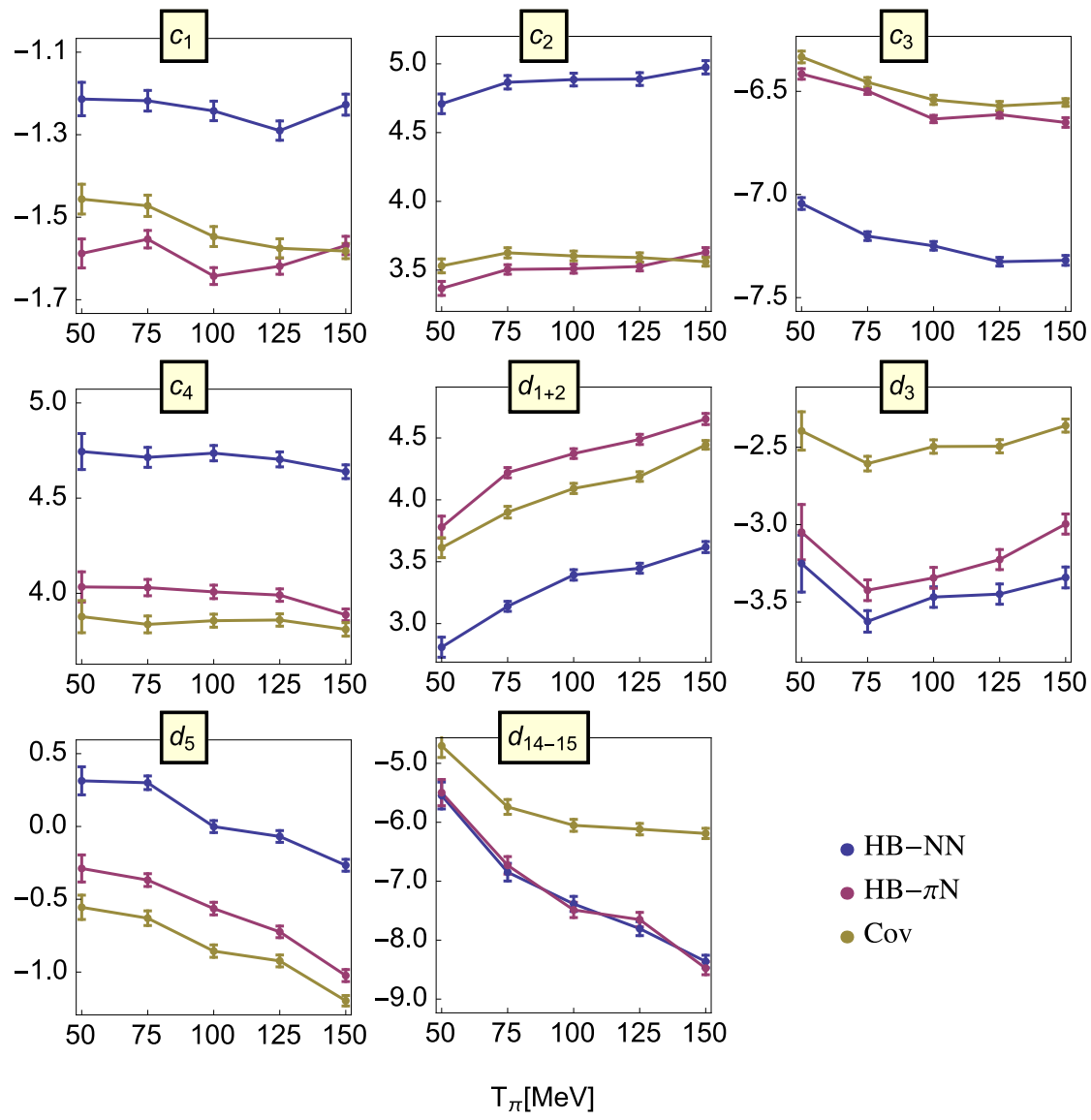

- $\mathrm{HB}-\mathrm{NN}$

- $\mathrm{HB}-\pi \mathrm{N}$

- $\mathrm{Cov}$

FIG. 7: Change of LECs at $Q^{3}$ over maximum fit energy $T_{\pi}$. 

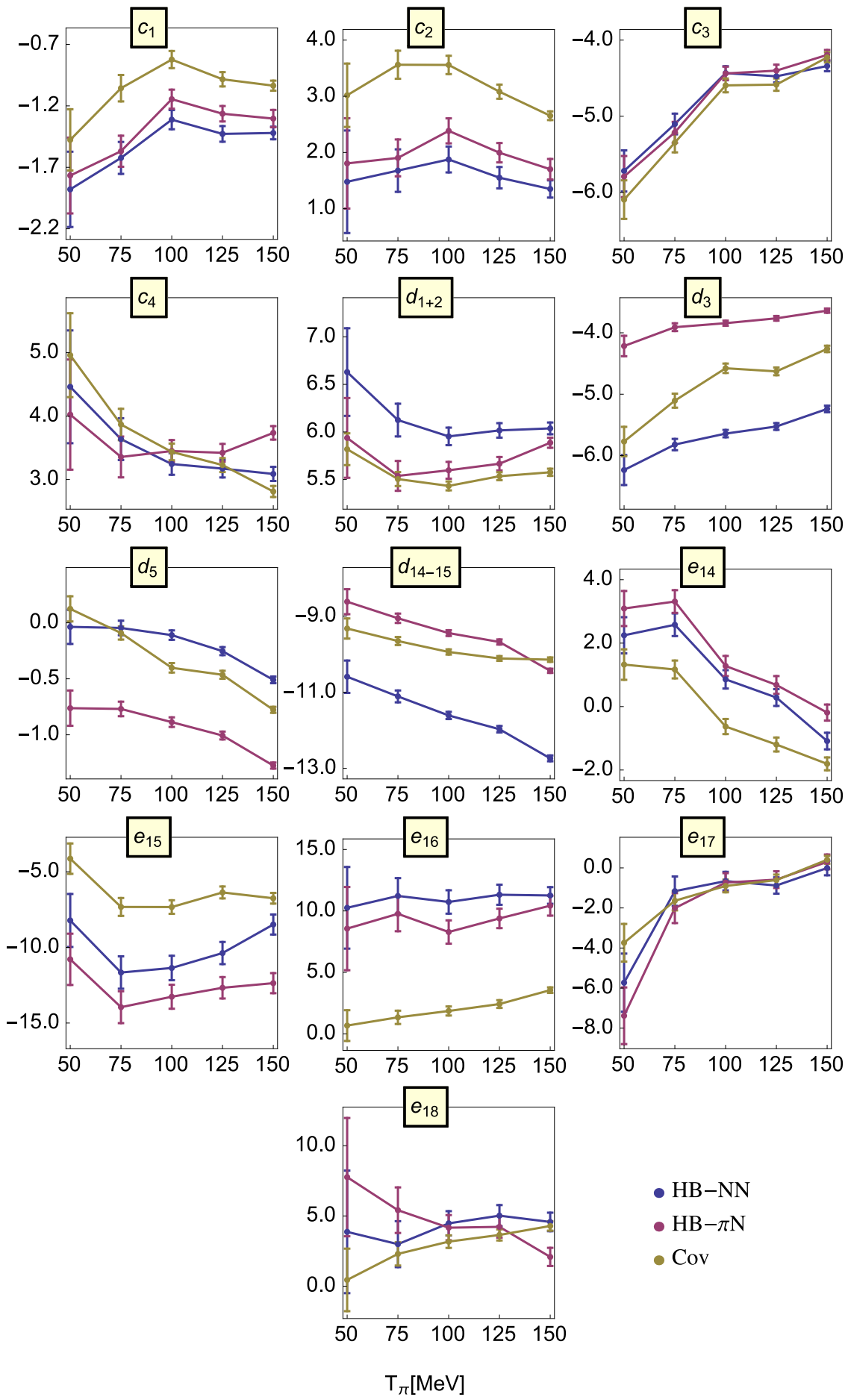

- $\mathrm{HB}-\mathrm{NN}$

- $\mathrm{HB}-\pi \mathrm{N}$

- $\mathrm{Cov}$

FIG. 8: Change of LECs at $Q^{4}$ over maximum fit energy $T_{\pi}$. 

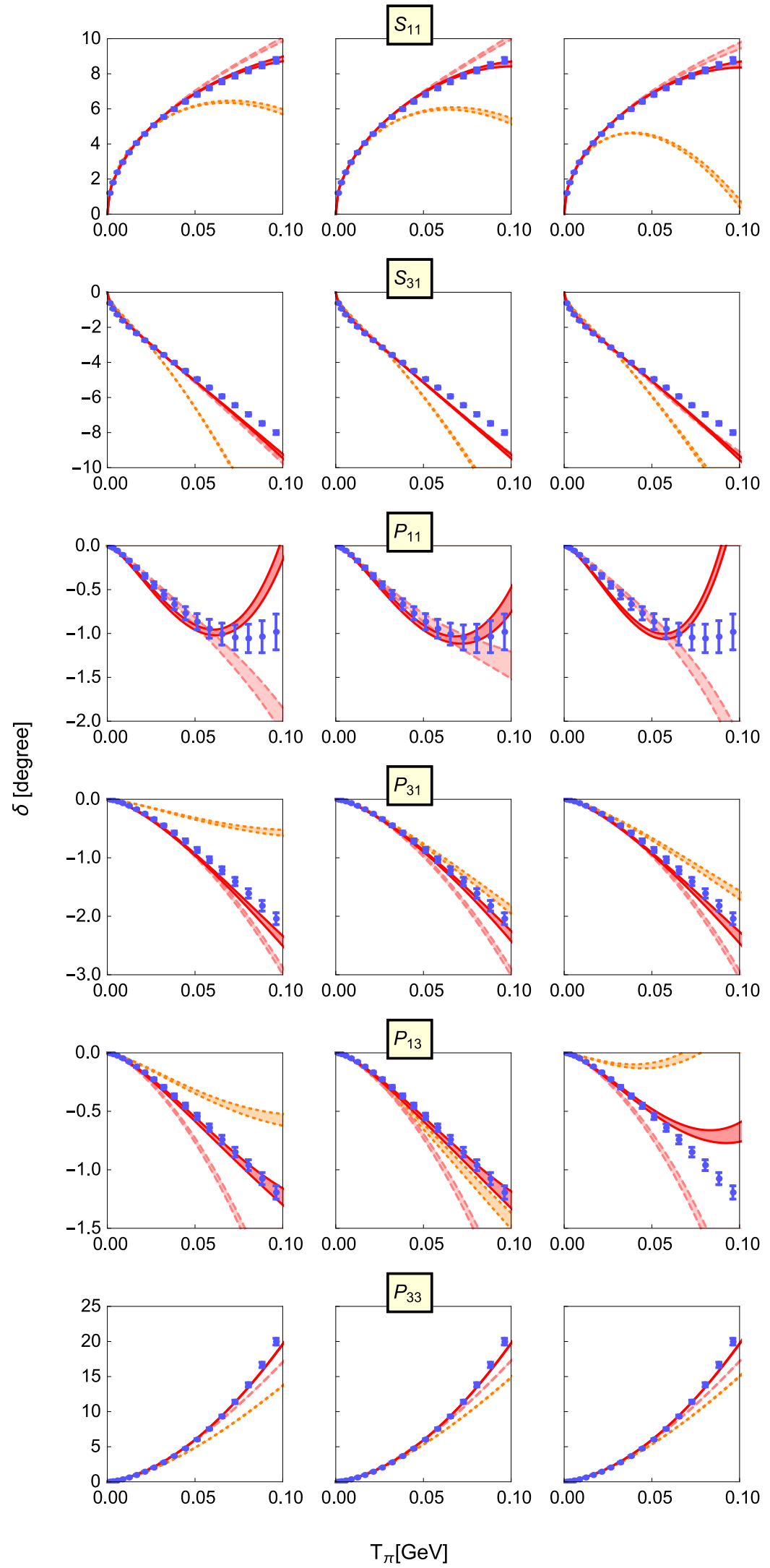

FIG. 9: (Color online) Predictions for $S$ waves up to $T_{\pi}=100 \mathrm{MeV}$. Columns from left to right corresponds to the to the predictions in the HB-NN, HB- $\pi \mathrm{N}$ and Covariant counting, respectively. The orange, pink and red (dotted, dashed and solid) bands refer to $Q^{2}, Q^{3}$ and $Q^{4}$ results including statistical uncertainties, respectively. 

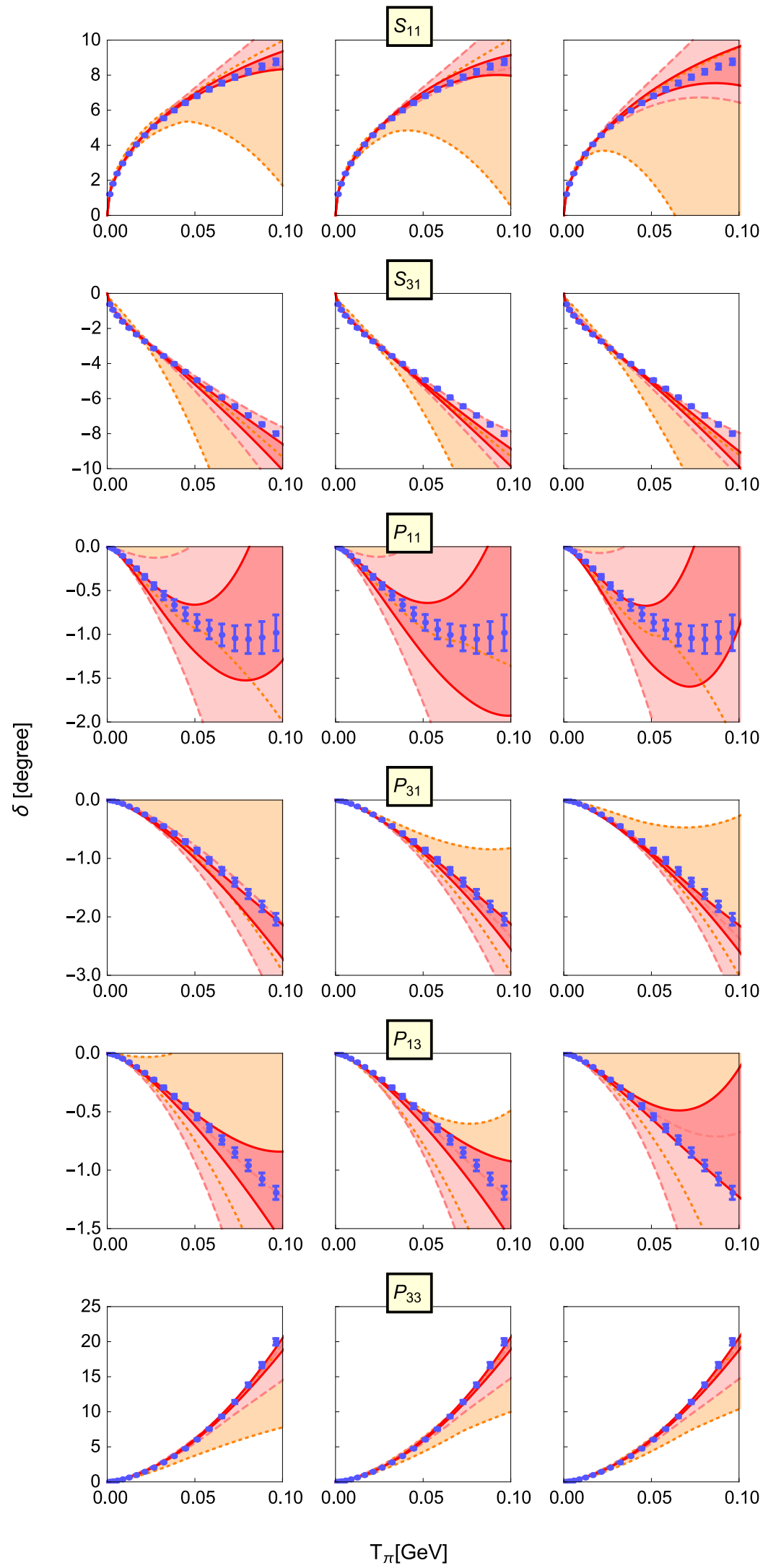

FIG. 10: (Color online) Predictions for $S$ waves up to $T_{\pi}=100 \mathrm{MeV}$. Columns from left to right corresponds to the to the predictions in the HB-NN, HB- $\pi \mathrm{N}$ and Covariant counting, respectively. The orange, pink and red (dotted, dashed and solid) bands refer to $Q^{2}, Q^{3}$ and $Q^{4}$ results including theoretical uncertainties, respectively. 

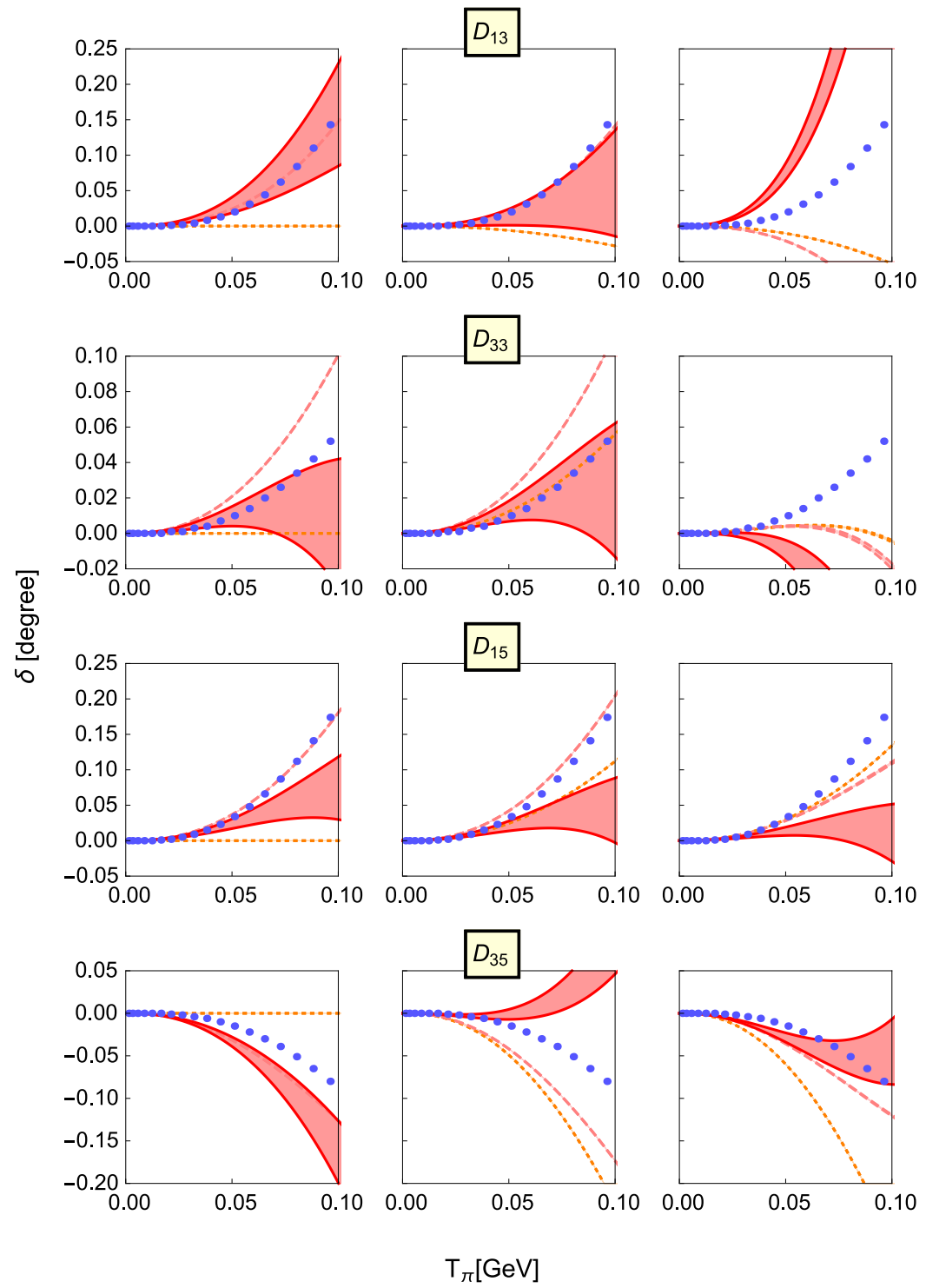

FIG. 11: (Color online) Predictions including statistical uncertainties for $D$ waves up to $T_{\pi}=$ $100 \mathrm{MeV}$. For remaining notation see Fig. 9 . 

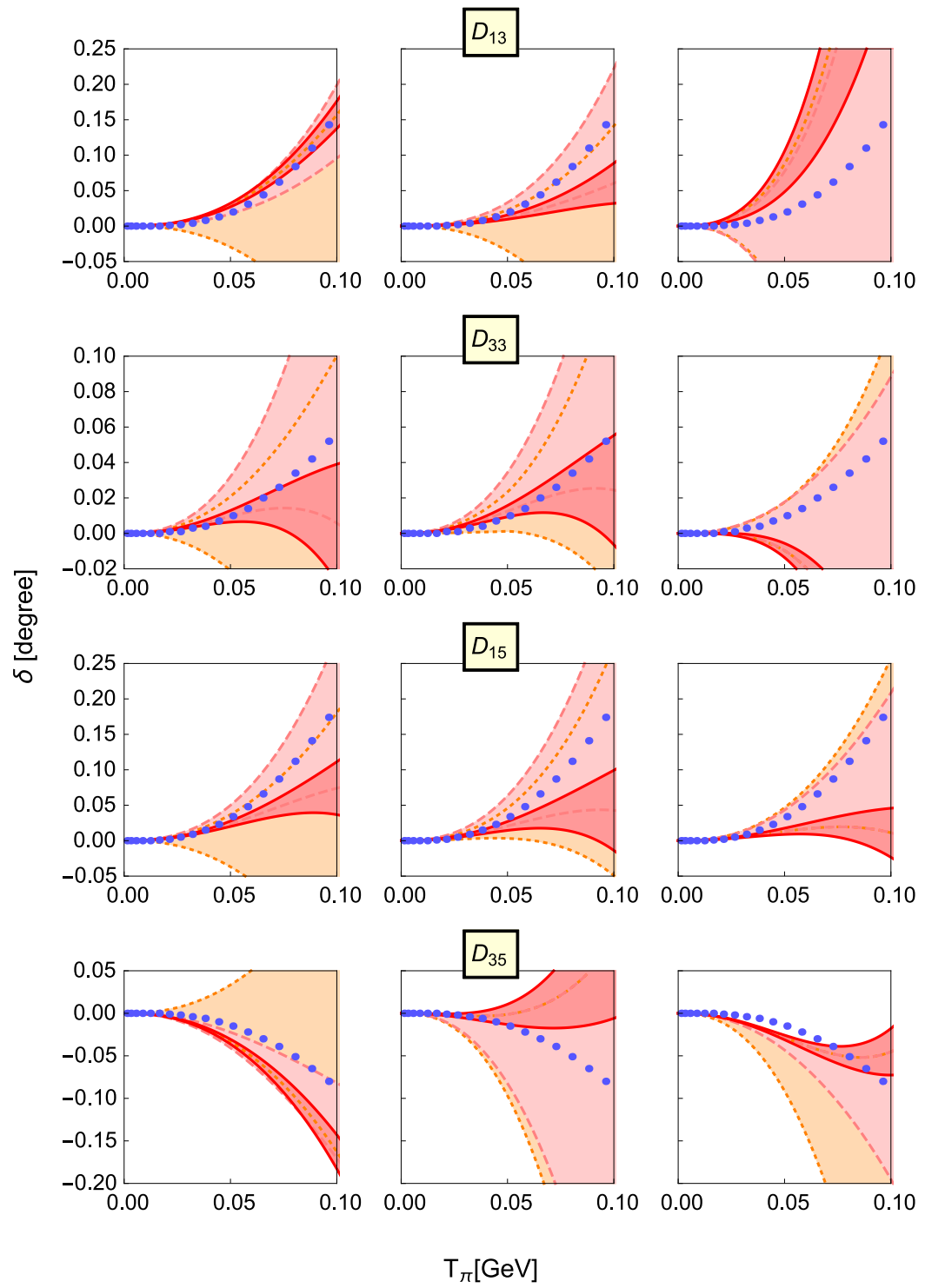

FIG. 12: (Color online) Predictions including theoretical uncertainties for $D$ waves up to $T_{\pi}=$ $100 \mathrm{MeV}$. For remaining notation see Fig. 10. 

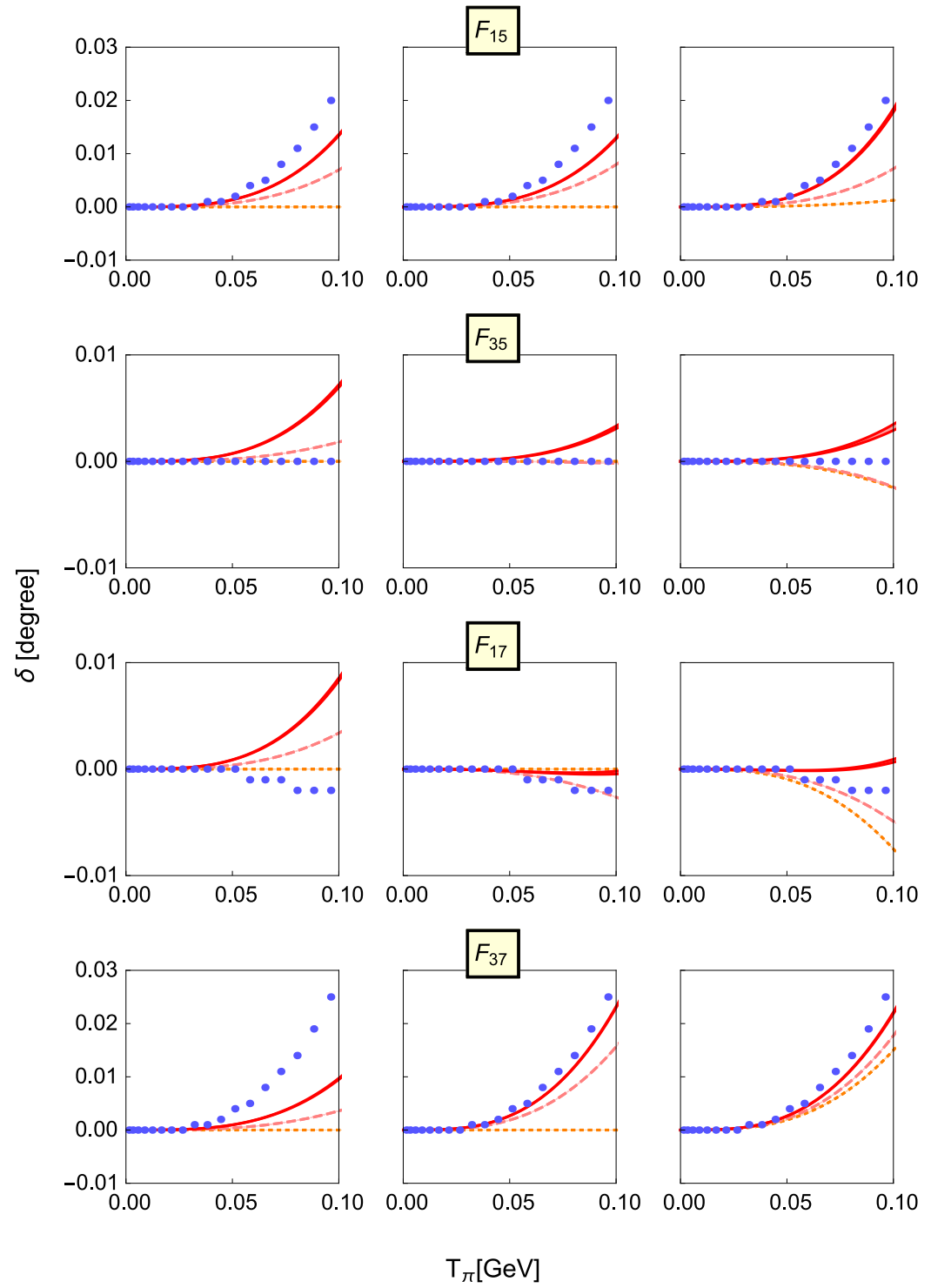

FIG. 13: (Color online) Predictions including statistical uncertainties for $F$ waves up to $T_{\pi}=$ $100 \mathrm{MeV}$. For remaining notation see Fig. 9 . 

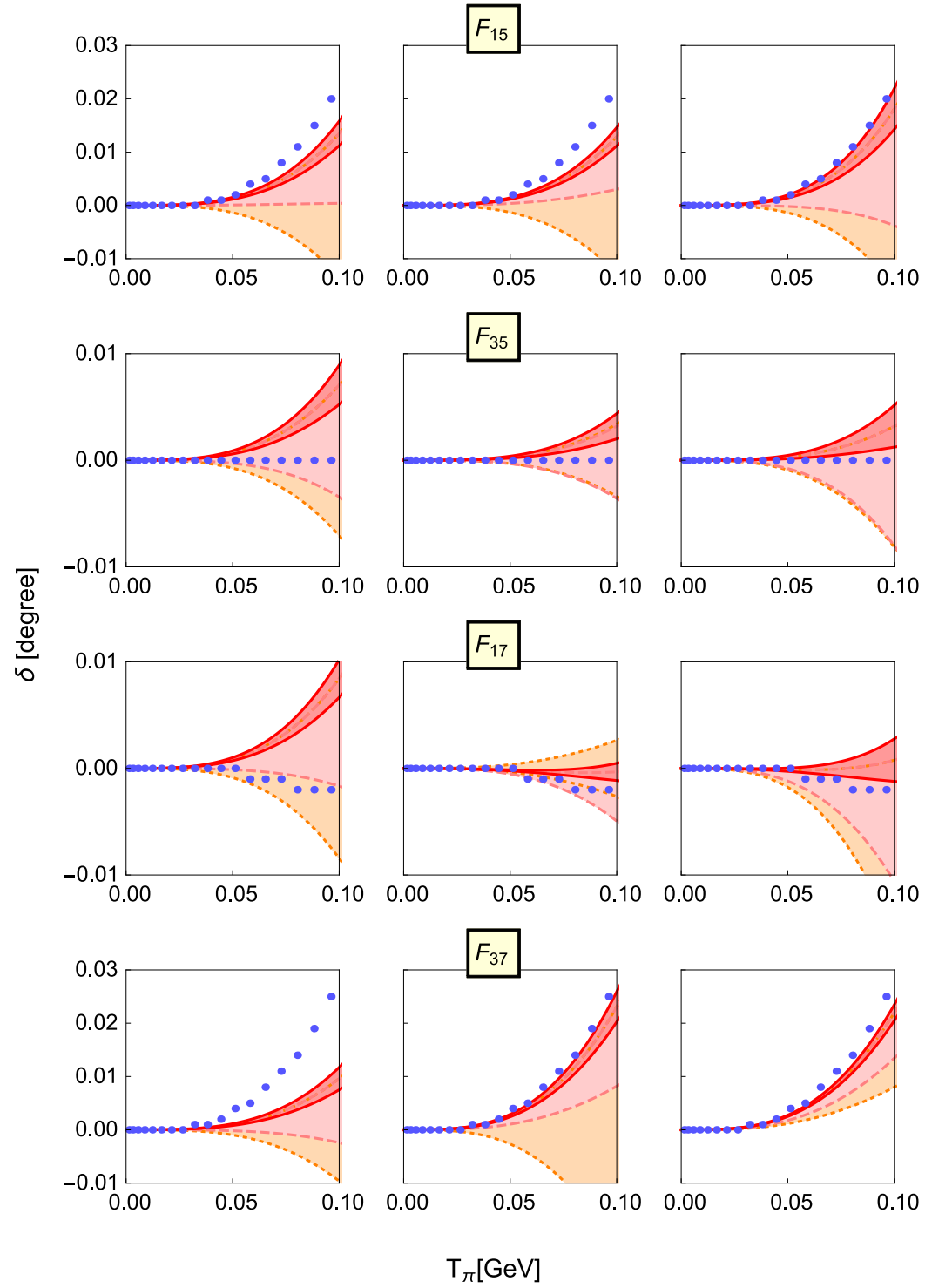

FIG. 14: (Color online) Predictions including theoretical uncertainties for $D$ waves up to $T_{\pi}=$ $100 \mathrm{MeV}$. For remaining notation see Fig. 10 . 

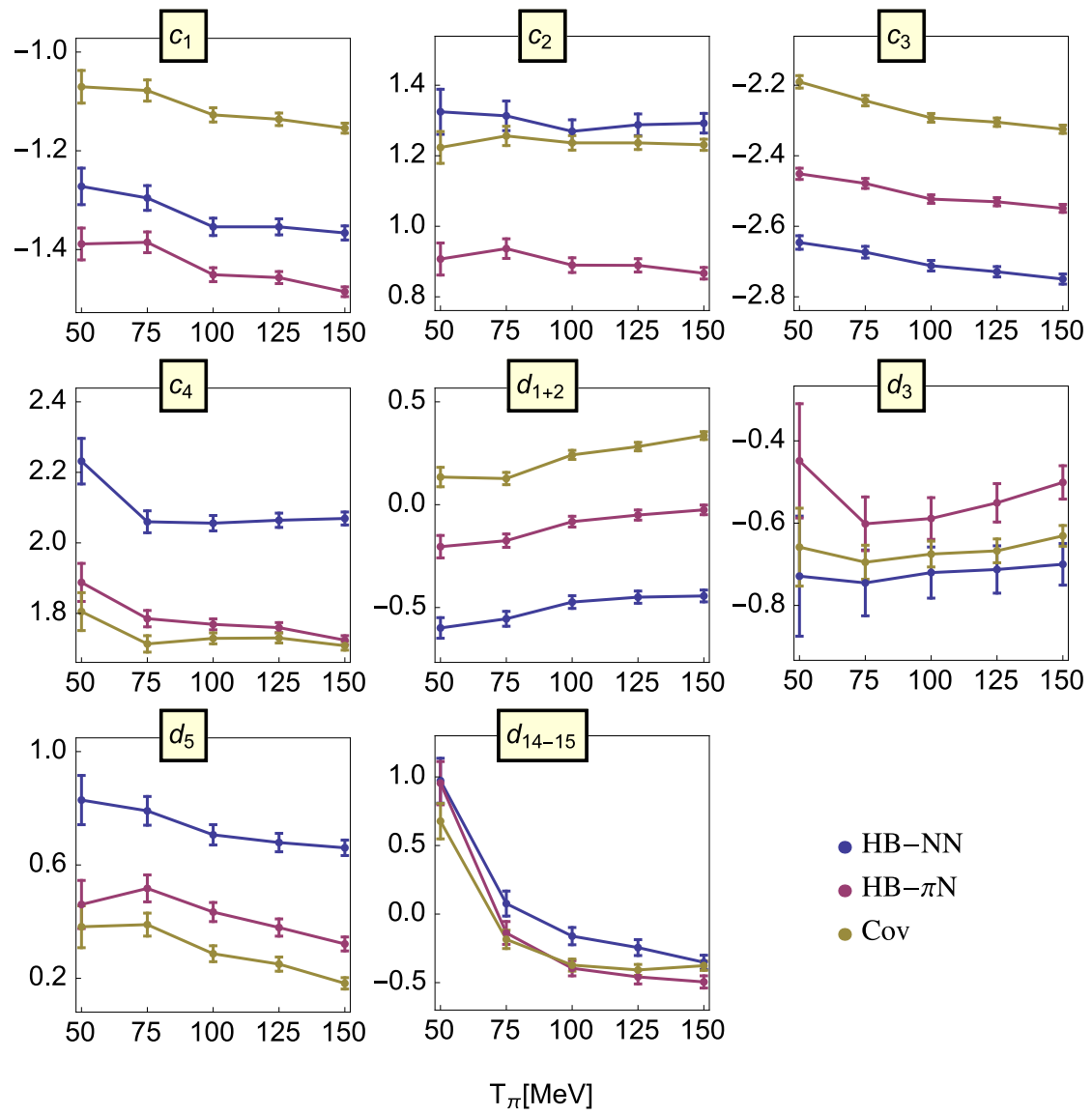

- HB-NN

- $\mathrm{HB}-\pi \mathrm{N}$

- $\mathrm{Cov}$

FIG. 15: Change of LECs at $Q^{3}+\delta^{1}$ over maximum fit energy $T_{\pi}$. 

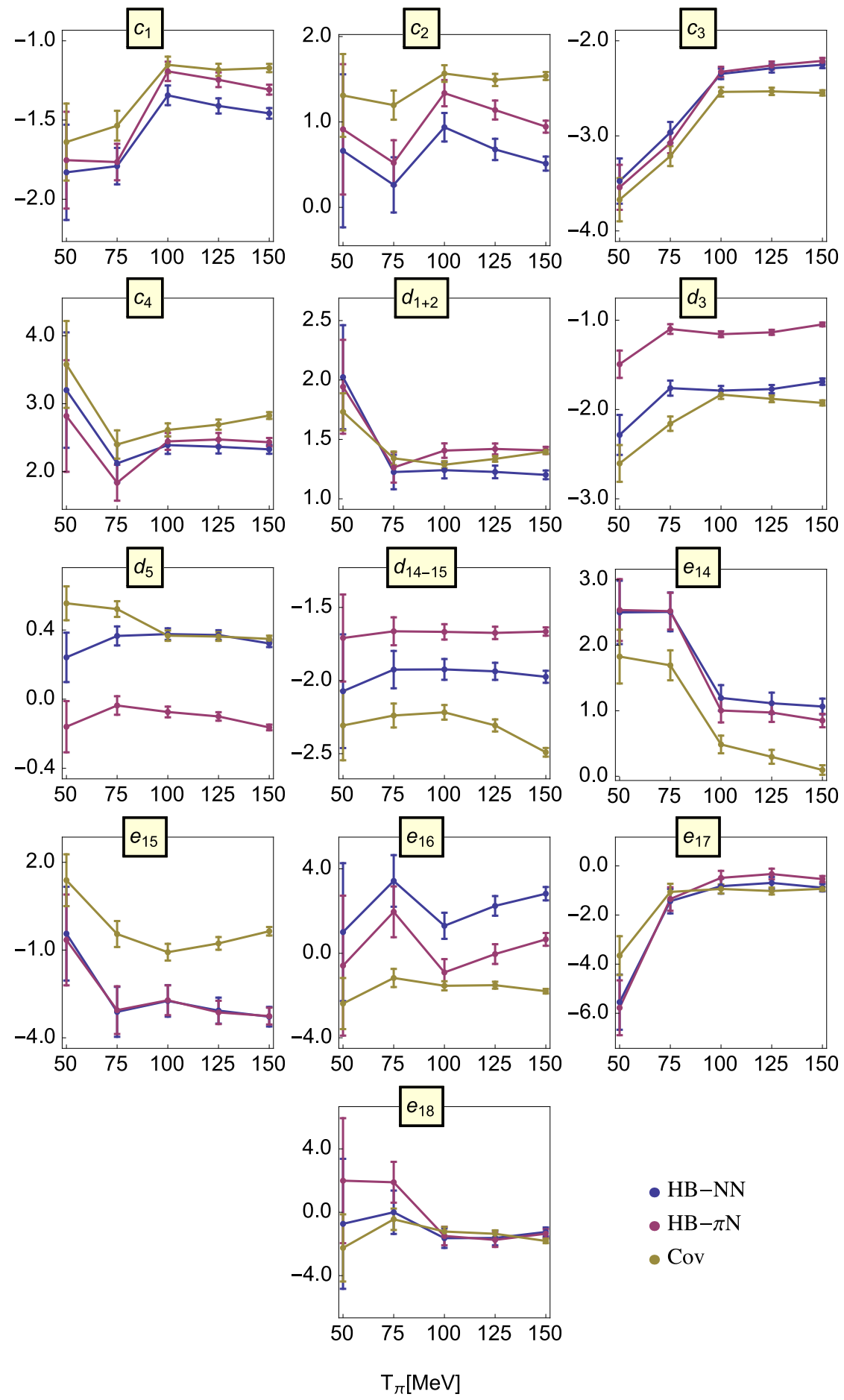

- $\mathrm{HB}-\mathrm{NN}$

- $\mathrm{HB}-\pi \mathrm{N}$

- $\mathrm{Cov}$

FIG. 16: Change of LECs at $Q^{4}+\delta^{1}$ over maximum fit energy $T_{\pi}$. 

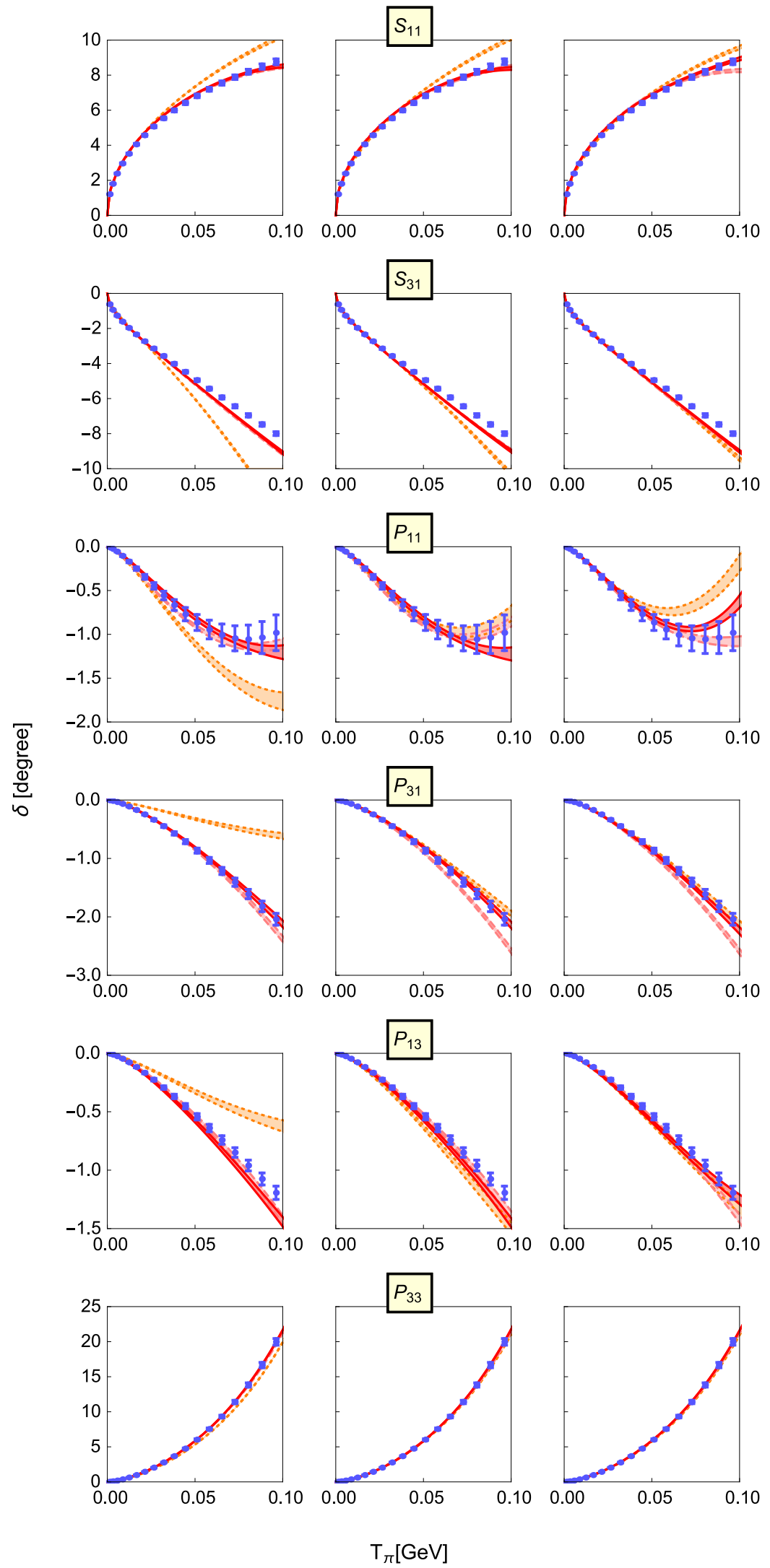

FIG. 17: (Color online) Predictions for $S$ waves up to $T_{\pi}=100 \mathrm{MeV}$. Columns from left to right corresponds to the to the predictions in the HB-NN, HB- $\pi \mathrm{N}$ and Covariant counting, respectively. The orange, pink and red (dotted, dashed and solid) bands refer to $Q^{2}+\delta^{1}, Q^{3}+\delta^{1}$ and $Q^{4}+\delta^{1}$ results including statistical uncertainties, respectively. 

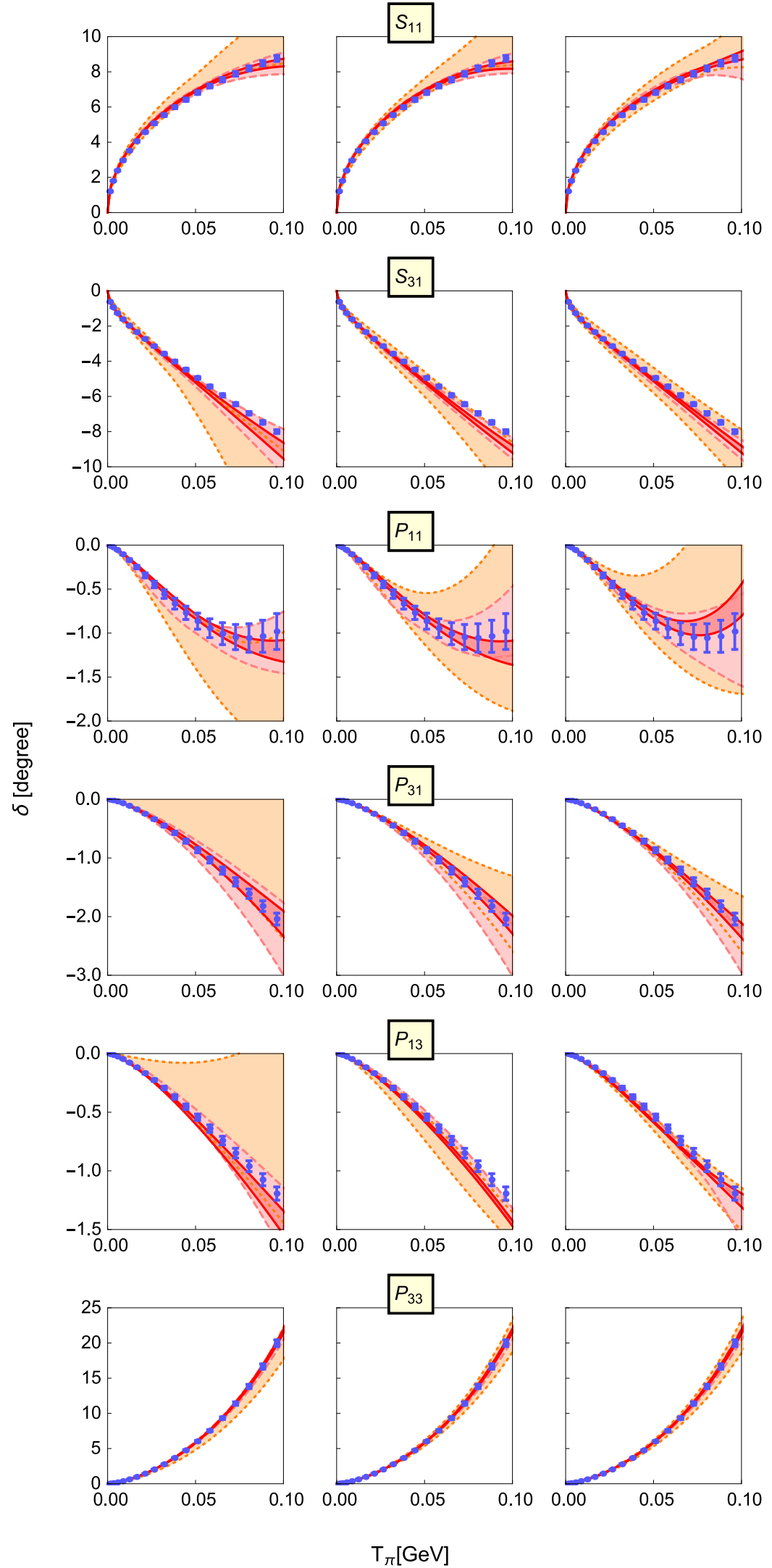

FIG. 18: (Color online) Predictions for $S$ waves up to $T_{\pi}=100 \mathrm{MeV}$. Columns from left to right corresponds to the to the predictions in the HB-NN, HB- $\pi \mathrm{N}$ and Covariant counting, respectively. The dotted, dashed and solid bands refer to $Q^{2}+\delta^{1}, Q^{3}+\delta^{1}$ and $Q^{4}+\delta^{1}$ results including theoretical uncertainties, respectively. 

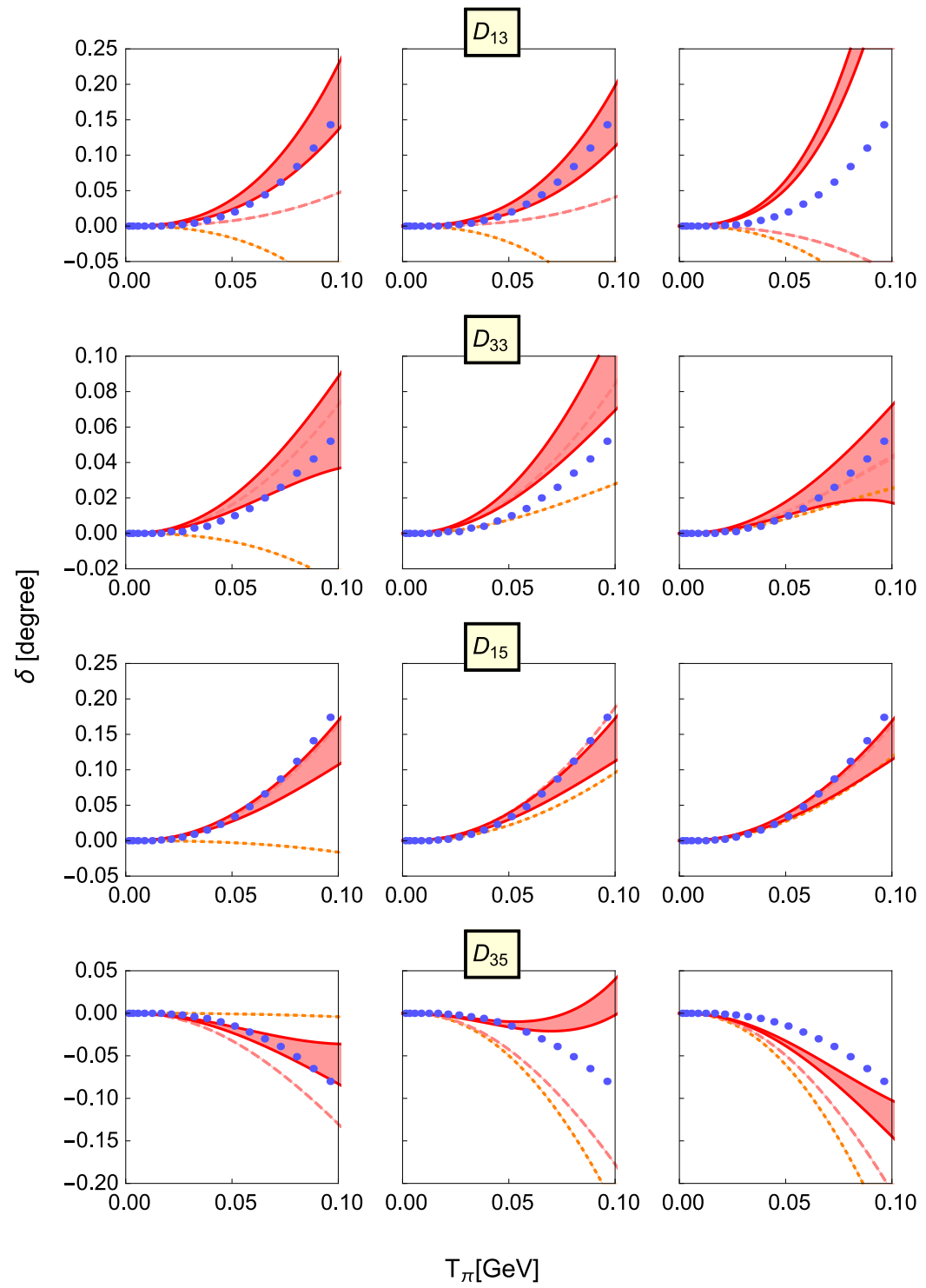

FIG. 19: (Color online) Predictions including statistical uncertainties for $D$ waves up to $T_{\pi}=$ $100 \mathrm{MeV}$. For remaining notation see Fig. 17. 

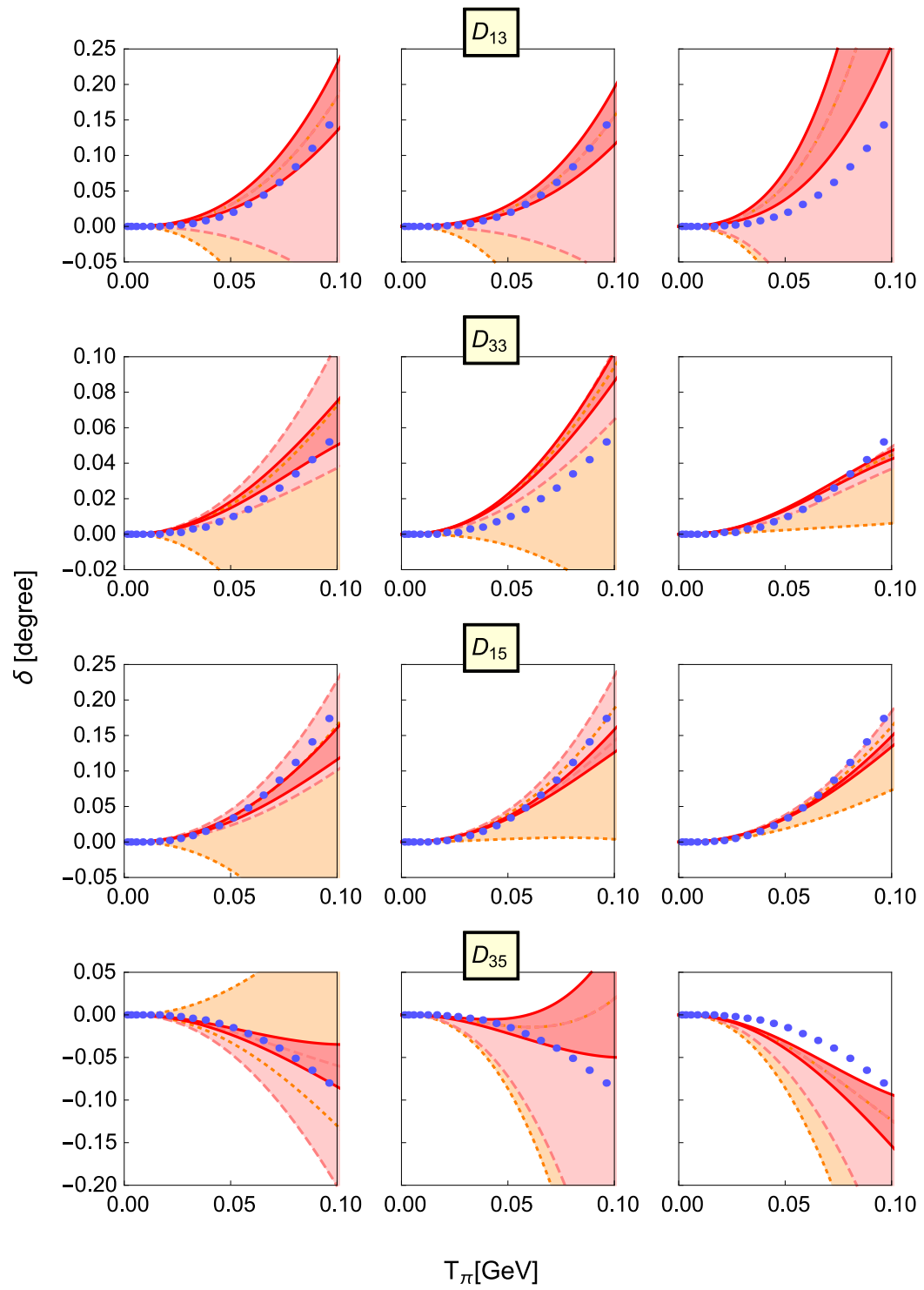

FIG. 20: (Color online) Predictions including theoretical uncertainties for $D$ waves up to $T_{\pi}=$ $100 \mathrm{MeV}$. For remaining notation see Fig. 18. 

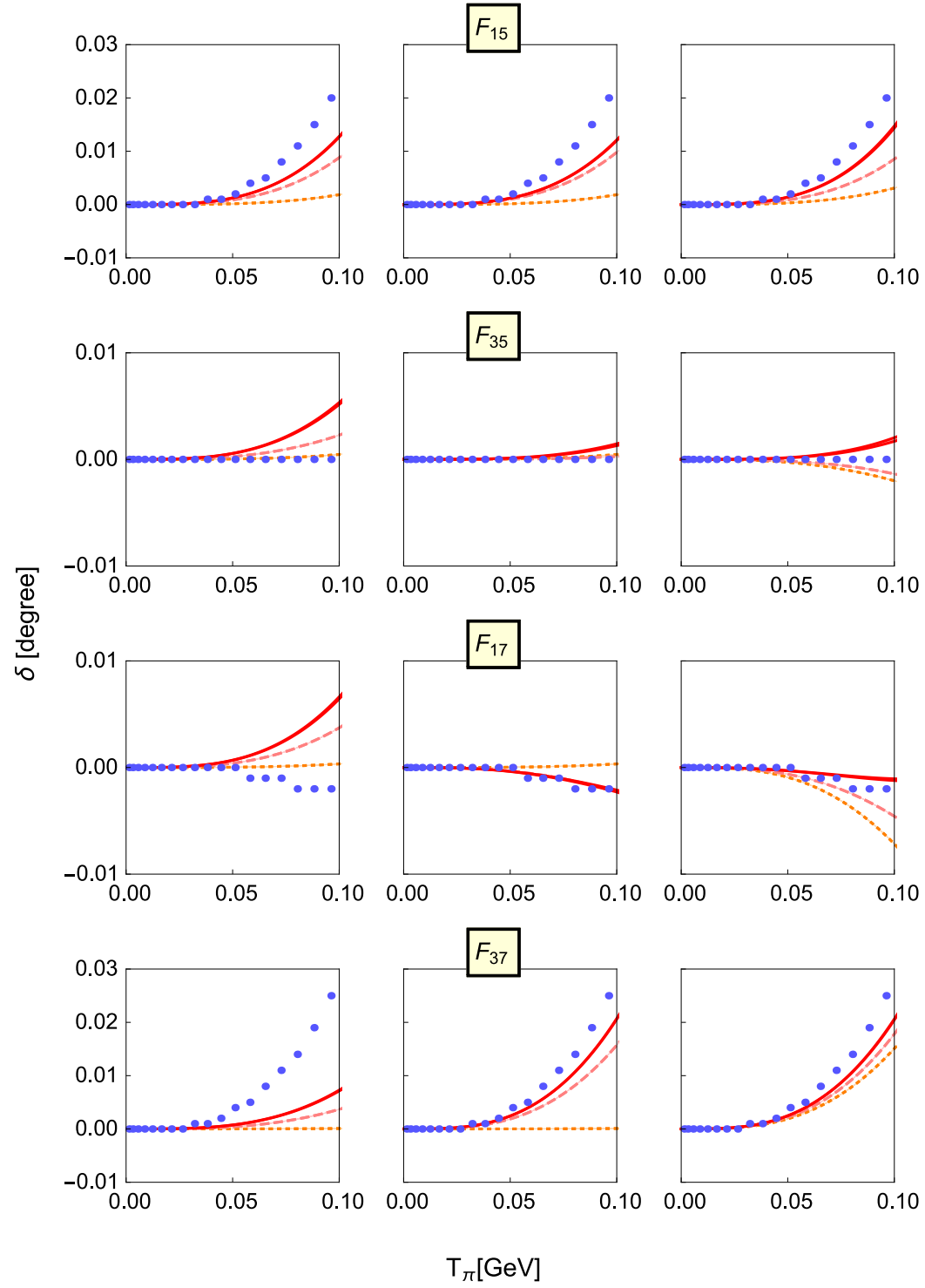

FIG. 21: (Color online) Predictions including statistical uncertainties for $F$ waves up to $T_{\pi}=$ $100 \mathrm{MeV}$. For remaining notation see Fig. 17. 

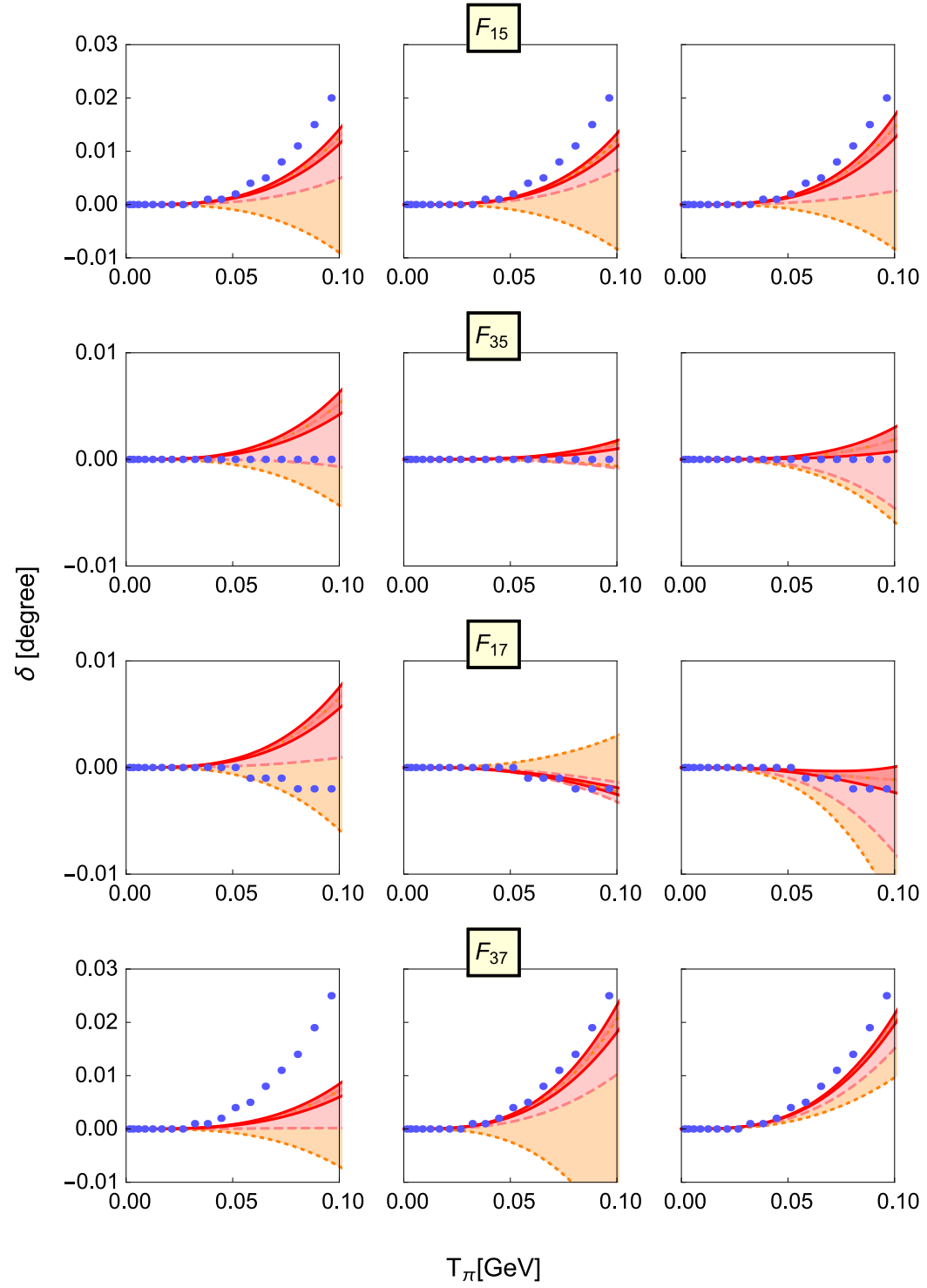

FIG. 22: (Color online) Predictions including theoretical uncertainties for $D$ waves up to $T_{\pi}=$ $100 \mathrm{MeV}$. For remaining notation see Fig. 18 . 


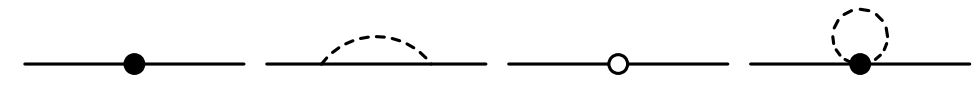

FIG. 23: Diagrams contributing to the nucleon self energy. For notation see Fig. 1.
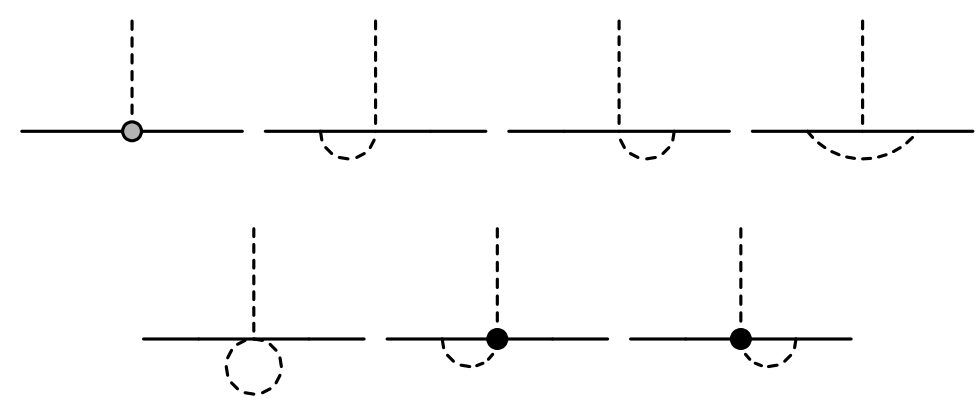

FIG. 24: Diagrams contributing to the axial vector coupling of the nucleon. For notation see Fig. 1. 